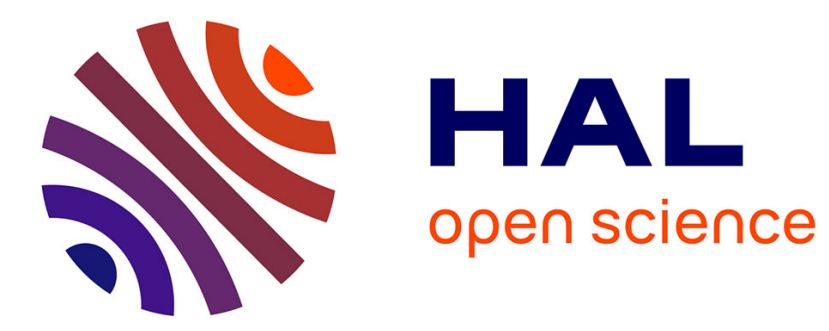

\title{
Resolving hydromechanical coupling in two and three dimensions: spontaneous channelling of porous fluids owing to decompaction weakening
}

L Räss, Thibault Duretz, Y. Podladchikov

\section{- To cite this version:}

L Räss, Thibault Duretz, Y. Podladchikov. Resolving hydromechanical coupling in two and three dimensions: spontaneous channelling of porous fluids owing to decompaction weakening. Geophysical Journal International, 2019, 218 (3), pp.1591-1616. 10.1093/gji/ggz239 . insu-02284570

HAL Id: insu-02284570

https://hal-insu.archives-ouvertes.fr/insu-02284570

Submitted on 12 Sep 2019

HAL is a multi-disciplinary open access archive for the deposit and dissemination of scientific research documents, whether they are published or not. The documents may come from teaching and research institutions in France or abroad, or from public or private research centers.
L'archive ouverte pluridisciplinaire HAL, est destinée au dépôt et à la diffusion de documents scientifiques de niveau recherche, publiés ou non, émanant des établissements d'enseignement et de recherche français ou étrangers, des laboratoires publics ou privés. 


\title{
Resolving hydromechanical coupling in two and three dimensions: spontaneous channelling of porous fluids owing to decompaction weakening
}

\author{
L. Räss ${ }^{\oplus}, 1,2$ T. Duretz ${ }^{\oplus 1,3}$ and Y.Y. Podladchikov ${ }^{\oplus 1,2}$ \\ ${ }^{1}$ Institut des Sciences de la Terre, Faculté des géosciences et de l'environnement, Université de Lausanne, Lausanne, Switzerland. \\ E-mail: ludovic.rass@gmail.com \\ ${ }^{2}$ Swiss Geocomputing Centre, Université de Lausanne, Lausanne, Switzerland \\ ${ }^{3}$ Géosciences Rennes, Univ. Rennes 1, UMR CNRS 6118, 35042 Rennes, France
}

\begin{abstract}
SUMMAR Y
Fingering, veining, channelling and focussing of porous fluids are widely observed phenomena in the Earth's interior, driving a range of geo-processes across all scales. While observations suggest fairly localized flow patterns induced by fractures, the classical Darcian model predicts diffusive behaviour that leads to never-ending spreading and delocalization. We here investigate an alternative physical mechanism without the need to involve fractures. Decompaction weakening leads to the formation and propagation of localized flow-pathways in fluid-saturated porous media. We numerically solve the coupled equations using high-resolution 2-D and 3-D numerical modelling to predict non-linear porous flow in a non-linearly viscously deforming matrix. We show that high-porosity channels may be a dynamic and natural outcome of sufficiently resolved hydromechanical coupling and decompaction weakening. We propose an efficient solution strategy that involves an iterative pseudo-transient numerical method to solve the coupled system of equations in a matrix-free fashion. We discuss benefits and limitations of this approach that performs optimally on hardware accelerators such as graphical processing units and is well-suited for supercomputing. We benchmark the pseudo-transient routines against commonly used direct-iterative solving strategies and show convergence towards identical results. Furthermore, we use the fast solver to systematically study in 2-D the high-porosity channel propagation velocity as a function of bulk and shear viscosity ratios and report discrepancy between 2-D and 3-D configurations. We conclude that the fluid-flow rate in the channels is up to three orders of magnitude higher than expected by pure Darcian flow regimes and show that the high-porosity channels occurrence remains with strain rate dependant shear viscosity. We provide both the 2-D MATLAB-based direct-iterative and pseudo-transient routines for full reproducibility of the presented results and suggest our model configuration as a key benchmark case to validate the implementation of hydromechanical coupling in 2-D and 3-D numerical codes. The routines are available from Bitbucket and the Swiss Geocomputing Centre website, and are also supporting information to this paper.
\end{abstract}

Key words: Creep and deformation; Permeability and porosity; Geomechanics; Non-linear differential equations; Numerical modelling.

\section{INTRODUCTION}

\subsection{Subsurface flow localization}

Evidence of localized fluid flow in saturated porous media are observed in various geosystems on Earth. Their expression in the interior of deep Earth relates to metasomatism and dehydration of aqueous minerals in ductile rocks (Connolly \& Podladchikov 2000, 1998;
Miller et al. 2003; Ague 2011; Iyer et al. 2013; Skarbek \& Rempel 2016; Omlin et al. 2017a) and migration of melt into deforming partially molten regions of the lithosphere (McKenzie 1984; Scott \& Stevenson 1984; Connolly \& Podladchikov 2007; Simpson et al. 2010a,b; Liang et al. 2011; Rudge et al. 2011; Schiemenz et al. 2011; Katz \& Weatherley 2012; Cai \& Bercovici 2013; Keller et al. 2013, 2017). At shallower levels, vertical chimneys or pipe structures are populating sedimentary basins in continental shelves (Judd 
$\&$ Hovland 2007). These structures are inferred from their distinct signatures on seismic cross-sections and are often related to pockmarks on the seafloor. Their presence is especially well documented in regions of economic interest (Berndt 2005; Cathles et al. 2010; Räss et al. 2014; Cartwright \& Santamarina 2015; Minakov et al. 2017; Elenius et al. 2018). Indications suggest that some vertical pipes may act as preferential fluid pathways, while other structures appear to be dormant. The expression of these subseabed pipes is well reported for instance on the Nigerian continental shelf and in the Norwegian North Sea (Hustoft et al. 2010; Plaza-Faverola et al. 2010; Løseth et al. 2011; Plaza-Faverola et al. 2011; Mazzini et al. 2017; Huq et al. 2017), as well as in lacustrine environments (Reusch et al. 2015). Understanding the physical process that leads to the formation and evolution of these pipes is essential to accurately constrain subsurface fluid flow. Most field observations are qualitatively explained by conceptual models, and only a few recent studies address the physics of gas-rich and fluid-rich chimneys (e.g. Cathles et al. 2010; Cartwright \& Santamarina 2015, and references therein); however, they mainly involve capillary forces and fracture mechanics that lead to a potential blowout scenario. An alternative mechanism suggests that these subsurface channel-like flow features are the result of localized creep of the porous matrix coupled to non-linear Darcian flow (Räss et al. 2018; Böttner et al. 2019). The solitary waves limit of hydromechanically coupled model provides in this case the underlying physical mechanism leading to the occurrence of flow instability in viscously deforming porous media (Räss et al. 2018).

\subsection{Multiphysics coupling}

To date, most porosity wave models (Barcilon \& Richter 1986; O1son \& Christensen 1986; Scott 1988; Wiggins \& Spiegelman 1995; Connolly \& Podladchikov 1998, 2007, 2000; Appold \& Nunn 2002; Tian \& Ague 2014; Joshi \& Appold 2016; Skarbek \& Rempel 2016) decouple fluid flow from shear deformation by assuming lithostatic total pressure gradient. The fully coupled model utilizes appropriate geomechanics to predict stresses and pressure distribution in the porous matrix. The fully coupled approach has two major benefits; first, total pressure no longer needs to be assumed to follow a lithostatic gradient; secondly, the shear deformation of the porous matrix is resolved. Most importantly, the dynamics and morphology of porosity waves become sensitive to the shear rheology of the rock matrix. The fully coupled approach's importance has been discussed concerning industry-related simulators based on Biot's poro-elasticity theory (Settari et al. 2001; Prevost 2013). There, the standard solution to simulate fluid-flow in porous media relies on a direct coupling approach, in which fluid pressure is transferred to the geomechanical module, but the geomechanics often do not impact the fluid pressure. Such an approach does not include any non-linear iteration, mainly owing to time constrains, and results in an explicit coupling strategy. More advanced workflows iteratively couple a fluid-flow solver to a geomechanical solver (e.g. Minkoff et al. 2003; Rutqvist 2012, 2011). In such an approach, data must often be explicitly transferred from one software package to another, hindering the maximal affordable numerical resolution. Both solution strategies often do not ensure non-linear convergence, owing to the large number of iterations needed, resulting in long simulation times. The computed solutions may not be accurate, and non-linear features may be under-resolved. An alternative solution is to fully couple fluid flow and Stokes matrix flow in a single solver (Scott 1988; Stevenson \& Scott 1991; Morency et al. 2007; Gradmann et al. 2012; Gradmann \& Beaumont 2012; Dymkova \& Gerya 2013; Keller et al. 2013; Dannberg \& Heister 2016; Zheng et al. 2016; Omlin et al. 2017b). Further, none of the previous studies, with the exception of Omlin et al. (2017b), have considered decompaction weakening while coupling Darcian and Stokes flows in 3-D.

\subsection{Previous work}

Yarushina \& Podladchikov (2015a) presented a general system of thermodynamically admissible equations (i.e. a system that ensures non-negative entropy production) for fluid flow in deformable viscoelastic porous media. The well-established Biot poro-elastic model (Wang 2000) is recovered in the linear elastic limit, valid at low stress levels and negligible viscous relaxation. A number of numerical studies (e.g. Phillips \& Wheeler 2008; Haga et al. 2012; Wheeler et al. 2014, and references therein), have explored linear poro-elastic coupling. At higher stress levels or temeprature, rock deformation deviates significantly from a linear elastic rheology. The non-linear instantaneous response is typically characterized using an elasto-plastic rheology (Baud et al. 2000; Vajdova et al. 2012; Wong \& Baud 2012). Lewis \& Schrefler (1987) and more recently Cacace \& Jacquey (2017) or Wheeler et al. (2014) numerically investigated poro-elasto-plastic deformation coupled to fluid flow. Pure elastic and elasto-plastic matrix rheologies are beyond this study's scope. We will neither address reactive transport modelling and thus not investigate chemical or thermal (energetic) feedbacks. Thermal and chemical couplings to deformation (mechanics) represent key processes to integrate in future fully coupled thermo-hydro-chemico-mechanical (THCM) models; as reported in well documented studies (Aharonov et al. 1996, Spiegelman \& Kelemen 2003; Liang et al. 2011; Oliveira et al. 2017). For exemple, hydrochemical coupling may trigger the generation of porosity from an initially non-porous rocks due to reactions that liberate fluid excesses which turn out to propagate in high-porosity channels (Malvoisin et al. 2015; Omlin et al. 2017a; Plümper et al. 2017). As a general trend, coupling among processes triggers nonlinear interactions that may result in significant and spontaneous localization of flow, heat and deformation (e.g. Duretz et al. 2019).

\subsection{The proposed approach}

In this study, we explore the viscous limit of Yarushina \& Podladchikov (2015a) model to quantify the importance of decompaction weakening while coupling Darcian and Stokes flows (HM coupling). Viscous rheology is expected to operate in the rock matrix at high (comparable to melting) temperatures and is usually neglected in lower-temperature environments. However, even at low (ambient) temperatures, rock deformation experiments on sandstones, limestones and shales show that major reservoir rock types exhibit nonnegligible time-dependent creep deformation (Spiers et al. 1990; Brantut et al. 2013; Sone \& Zoback 2014; David et al. 2015). Recent studies (Makhnenko \& Labuz 2016; Räss et al. 2017b) suggest that the porosity-dependent bulk viscosity is a non-linear function of the effective pressure. This strong non-linear interaction may lead to a drastic decrease in bulk viscosity values for regions of elevated pore-fluid pressure. Thus, the viscosity drop from the compaction to the decompaction regime, the decompaction weakening, suggested on theoretical grounds (Connolly \& Podladchikov 2007; Yarushina \& Podladchikov 2015a) is thus experimentally confirmed (Makhnenko \& Podladchikov 2018). 
We use numerical modelling to resolve prediction, rather than prescription, of the spontaneous formation and propagation of pipe structures in viscously deforming porous media (Keller et al. 2013; Räss et al. 2014, 2017b, 2016; Omlin et al. 2017b). Solitary waves require non-linear porosity-dependent permeability, here a CarmanKozeny relationship (Costa 2006) in order to form blob-shaped propagating porosity waves, but not yet reproducing the first-order natural data. Decompaction weakening results in the blobs to channels transition in better agreement with field observations. Such numerical models are computationally challenging because (1) the spatial occurrence of these structures is not known in advance; (2) the material properties such as permeability and viscosity range over several orders of magnitude and (3) important contrasts in spatial and temporal scales must be resolved, ranging from channel spacing down to the decompacting area at the tip of each propagating channel (Räss et al. 2018). Thus, the strong non-linear coupling among the different physical processes requires appropriate numerical treatment. The computational domain size must be large enough to resolve spontaneous spacing between the channels, and the numerical resolution must be sufficiently high to resolve the channel's tip areas mandatory for accurate predictions of the propagation velocity and growth of the channels.

We use the finite-difference method with two different solving approaches, a direct-iterative (DI) method (Newton and Picard linearizations) and a matrix-free pseudo-transient (PT) approach. We perform both 2-D and 3-D forward simulations and present a systematic study based on more than 400 forward 2-D simulations at various high resolutions and an extremely high-resolution 3D simulation involving more than 2 billion gridpoints. We suggest our model result as benchmark for future numerical two-phase poro-viscous implementations, augmenting the existing poro-elastic benchmark (Rozhko 2008) and the analytical solution of compacting flow past a sphere (Rudge 2014). We highlight that accurate convergence may lead to the formation of porosity waves and discuss the influence of the decompaction over compaction viscosity and the bulk to shear viscosity ratios in terms of maximal wave velocities. Furthermore, we report first results that the occurence of high-porosity channels remains while considering a strain-rate dependant power-law viscous shear rheology; we share potential implications those may trigger regarding subsurface processes. We finally discuss some technical insights that permit to optimally exploit modern hardware and address some performance-related considerations.

For reproducibility purpose, we provide the 2-D MATLAB PT and DI hydromechanical numerical codes. These MATLAB routines are part of M2Di (Räss et al. 2017a) and are available for download from Bitbucket at https://bitbucket.org//raess $/ \mathrm{m} 2 \mathrm{di}$ and from the Swiss Geocomputing Centre website http://www.unil.ch/geocom puting/software/. The PT routines (HM2Dpt) are located in the HM2Dpt folder and the DI routines (HM2Di) are located in the M2Di2 folder. The GPU (CUDA C + MPI) routines are available upon request to the authors.

\section{MATHEMATICAL MODEL AND HYDROMECHANICAL COUPLING}

We utilize a set of two-phase equations to model the formation and evolution of high-porosity channels over time as a natural outcome of the coupling of fluid flow and the deformation of a viscous porous matrix (Räss et al. 2014, 2018; Yarushina et al. 2015b). The mass balance for fluid and solid phases assuming constant fluid and solid densities as well as no fluid sources are:

$$
\begin{aligned}
\nabla_{k} v_{k}^{s} & =-\frac{D \log (1-\phi)}{D t}, \\
\nabla_{k}\left[\phi\left(v_{k}^{f}-v_{k}^{s}\right)\right] & =\frac{D \log (1-\phi)}{D t},
\end{aligned}
$$

where $\frac{D}{D t}=\frac{\partial}{\partial t}+v_{k}^{s} \nabla_{k}$ is the material derivative with respect to the solid.

The momentum balance equations for the matrix (Stokes) and the pore-fluid (Darcy) are:

$$
\begin{aligned}
\nabla_{j}\left(\bar{\tau}_{i j}-\bar{p} \delta_{i j}\right)-\bar{\rho} g_{i} & =0, \\
\phi\left(v_{i}^{f}-v_{i}^{s}\right)+\frac{k_{\phi}}{\mu^{f}}\left(\nabla_{i} p^{f}+\rho^{f} g_{i}\right) & =0,
\end{aligned}
$$

where $\bar{\tau}_{i j}$ are the components of the stress deviator, $\delta_{i j}$ is the Kronecker delta, $g_{i}$ are the components of downward-pointing gravity acceleration vector, $\phi\left(v_{i}^{f}-v_{i}^{s}\right)=v_{i}^{\text {Darcy }}$ is the Darcy flux vector (the relative flux of the fluid velocity $v_{i}^{f}$ relative to the solid velocity $\left.v_{i}^{s}\right), \mu^{f}$ is the pore-fluid viscosity and $\bar{p}, p^{f}$ are the total and fluid pressures, respectively. The total porosity-averaged density:

$\bar{\rho}=(1-\phi) \rho^{s}+\phi \rho^{f}$,

includes constant solid and fluid densities $\rho^{s}$ and $\rho^{f}$, respectively. The Carman-Kozeny relationship (Costa 2006) defines the porosity $\phi$ dependent permeability $k_{\phi}$ :

$k_{\phi}=k_{0}\left(\frac{\phi}{\phi_{0}}\right)^{n_{k}}$,

where $k_{0}$ is the reference permeability, $\phi_{0}$ the reference porosity and $n_{k}$ the permeability power law exponent.

The strain rate tensor and the viscous creep shear rheology of the solid are expressed as:

$\dot{\epsilon}_{i j}=\frac{1}{2}\left(\nabla_{i} v_{j}^{s}+\nabla_{j} v_{i}^{s}\right)-\frac{1}{3} \delta_{i j} \nabla_{k} v_{k}^{s}=\frac{1}{2 \mu_{s}} \bar{\tau}_{i j}$,

where $\dot{\epsilon}_{i j}$ is the strain rate tensor, $\delta_{i j}$ is the Kronecker-delta, $\mu_{s}$ is the solid shear viscosity and $\bar{\tau}_{i j}$ is the deviatoric stress tensor.

The system is closed by a final constitutive equation that accounts for viscous (de)compaction:

$\nabla_{k} v_{k}^{s}=-\frac{p_{e}}{\eta_{\phi}(1-\phi)}$,

where $p_{e}=\bar{p}-p^{f}$ is the effective pressure and $\eta_{\phi}$ is the bulk viscosity. The reference bulk compaction viscosity, $\eta_{\mathrm{c}}$, at reference porosity $\phi_{0}$ and $\bar{p}>>p^{f}$, is:

$\eta_{\mathrm{c}}=\frac{\mu_{s}}{C \phi_{0}}$

where $C$ is a rheological constant quantifying the ratio of shear viscosity over the reference bulk compaction viscosity. The rheological parameter $C$ may depend on the pore geometry and the effective pressure (Yarushina \& Podladchikov 2015a), but is assumed constant in this study as an approximation to more realistic compaction rheology.

At other porosity and fluid pressure values, the bulk viscosity $\eta_{\phi}$ is inversely proportional to the porosity and drops with the decrease of the effective pressure $p_{e}$ to account for decompaction weakening, here parametrized by a hyperbolic tangent function:

$\eta_{\phi}=\eta_{\mathrm{c}} \frac{\phi_{0}}{\phi}\left[1+\frac{1}{2}\left(\frac{1}{R}-1\right)\left(1+\tanh \left[-\frac{p_{e}}{\lambda_{p}}\right]\right)\right]$,

where $\lambda_{p}$ is the transition zone sharpness between the compacting and decompacting regime and $R=\eta_{\mathrm{c}} / \eta_{\mathrm{d}}$ is a rheological constant 
(Connolly \& Podladchikov 1998, 2007) quantifying the ratio of compaction $\left(\eta_{\mathrm{c}}\right)$ bulk viscosity (at $p_{e}>>\lambda_{p}$ ) over decompaction $\left(\eta_{\mathrm{d}}\right)$ bulk viscosity $\left(\right.$ at $\left.p_{e}<<-\lambda_{p}\right)$.

Within this study, we systematically vary the rheological constants $C$ and $R$ and investigate their impact on the flow morphology and velocity. We restrict our investigations to flow channelling cases where $R \geq 1$ and compare it to blob-like porosity waves at $R$ $=1$. The flow focussing mechanism we investigate here assumes a non-zero background porosity and saturates at some higher porosity $<<100$ per cent. We will thus not tackle the 0 per cent neither the 100 per cent porosity limits and stay below the suspension flow limit. This is in accordance with the two-phase flow mathematical model we rely on (Yarushina \& Podladchikov 2015a). Towards the 0 per cent porosity limit, effective permeability $k_{\phi} / \mu^{f} \rightarrow 0$ and bulk viscosity $\eta_{\phi} \rightarrow \infty$ leading to stagnation of the porosity waves. Wave trapping in the pure viscous limit can be avoided by including poro-elasticity (Connolly \& Podladchikov 1998) or chemical reactions (Malvoisin et al. 2015). Towards the 100 per cent porosity limit, the permeability and bulk viscosity models need modification in order to handle suspension flow regime. Both limits are beyond the scope of the current study. We also do not consider turbulent and other forms of non-Darcian porous fluid flow regimes (Skjetne \& Auriault 1999; Liu et al. 2015; Hawkins et al. 2016). Keller et al. (2013) further discuss details on two-phase flow model limits and their potential numerical treatments.

We use three independent scales:

$$
\begin{aligned}
\delta_{c} & =\sqrt{k_{0} \frac{\eta_{\mathrm{c}}}{\mu^{f}}}, \\
p_{c} & =\left(\rho^{s}-\rho^{f}\right) g \delta_{c}, \\
\tau_{c} & =\frac{\eta_{\mathrm{c}}}{p_{c}},
\end{aligned}
$$

and their dependent combinations such as the characteristic velocity $v_{c}=\delta_{c} / \tau_{c}$ to normalize all the variables, resulting in a dimensionless form of the governing equations. The characteristic length scale $\delta_{c}$ is also referred to as the compaction length (McKenzie 1984; Connolly \& Podladchikov 2014). $\tau_{c}$ is the characteristic time and $p_{c}$ is the characteristic pressure or stress.

Several non-linearities arise from the set of equations described above, both owing to coupling and porosity-dependent parameters. These non-linearities will require proper treatment in the solving procedure.

\section{MODEL CONFIGURATION}

The initial conditions we used in this study consist of a column of saturated porous media with an initial normalized background porosity value $\phi / \phi_{0}=1$. An elliptical region of $\phi / \phi_{0}=3$ is located at the first one-quarter above the computational domain's base. The computational 2-D or 3-D domain dimensions ( $x, h$ or $x, y, h$, respectively) are normalized by the characteristic length scale $\delta_{c}$ (Fig. 1). We report the main parameters used in this study, and not subject to change, in Table 1.

We apply free-slip boundary conditions for the Stokes problem (no shear stress and zero normal velocity on the boundaries). For the fluid flow part (i.e. the Darcy problem), we assign fixed flux boundary conditions; fluxes in $x$ and $y$ directions are set to 0 on all sides of the computational domain. Vertical inflow and outflow values (bottom and top boundaries) are chosen to satisfy the condition $p_{e}=$ 0 , leading to no compaction or decompaction of the porous matrix.

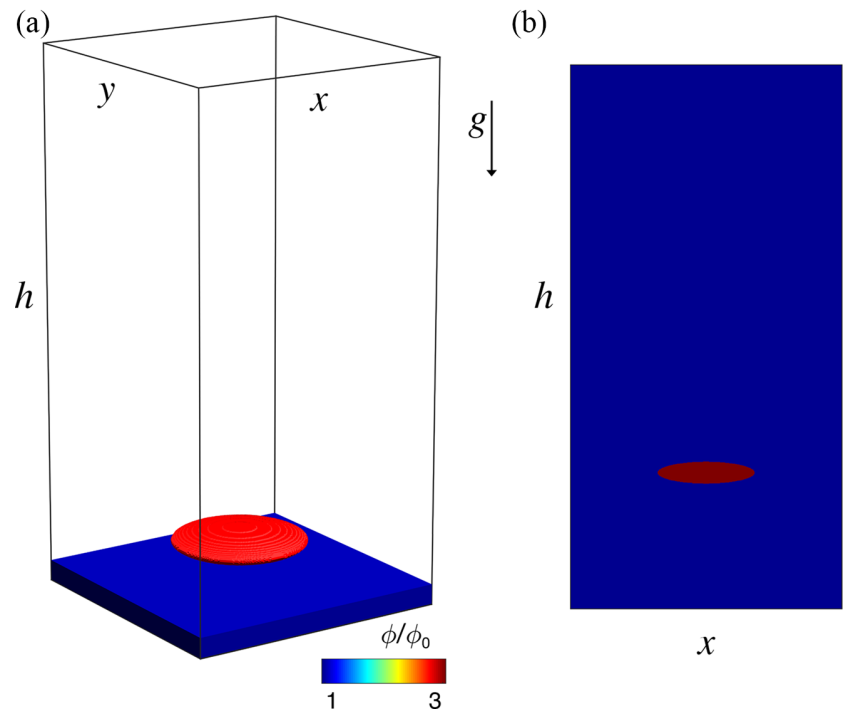

Figure 1. Schematic initial model configuration for (a) the 3-D and (b) the 2$\mathrm{D}$ calculations. The high-porosity anomaly is located at the first one-quarter of the model depth and the values are three times higher than background normalized porosity $\left(\phi / \phi_{0}\right)$.

Similar boundary condition types are used and further discussed in Rhebergen et al. (2015).

The viscous medium undergoes progressive compaction in response to the downward-pointing gravity acceleration. Since the fluid density is twice as low as the solid density, the fluid excess in the high-porosity anomaly tends to travel upwards as buoyancydriven flow. As further discussed in this study, the flow tends to focus in high-porosity channels if the decompaction weakening is significant, $\eta_{\mathrm{c}} / \eta_{\mathrm{d}}>>1$. The resulting flow rate and propagation velocities are therefore significantly enhanced. Fig. 2 shows three stages of a reference 2-D simulation of buoyancy-driven propagating porosity waves in a low-porosity region. For significant decompaction weakening, $\eta_{\mathrm{c}} / \eta_{\mathrm{d}}>>1$, the coupling of fluid flow and solid matrix deformation naturally rearranges into high-porosity channels. In this case, the resulting vertical fluid transfer rates are significantly higher than diffusive Darcian flow (Figs 2a-c). The corresponding effective pressure $p_{e}$ values show, at each stage, which part of the domain is in the compaction $\left(p_{e}>0\right)$ or decompaction $\left(p_{e}<0\right)$ regime (Figs $2 \mathrm{~d}-\mathrm{f}$ ). The high-porosity channels evolve and propagate upwards in a self-sustained mechanism, collecting the surrounding fluid via lower fluid pressure inside compared to outside the channels. Since mass is conserved, the regions surrounding the vertical channels compact owing to fluid depletion. The resulting increased bulk viscosity values in low-porosity regions turn out to freeze the channel wall geometry and potentially provide preferential flowpaths for future fluid release.

\section{NUMERICAL IMPLEMENTATION AND SOLVING STRATEGIES}

The system of partial differential equations (Section 2) that describes the two-phase poro-viscous flow is discretized using the finite difference method on a regular Cartesian staggered grid. This staggering is inherently devoid of oscillatory pressure modes (Shin \& Strikwerda 1997) and relies on second-order conservative finite differences (Patankar 1980; Virieux 1986; McKee et al. 2008). The standard Stokes staggered grid is augmented with fluid pressure 
Table 1. Non-dimensional simulation parameters. Notably, the 3-D configuration, $y$ is the second horizontal axis and is equal to the $x$-axis, which represents the domain width in 2-D and 3-D. Also, values identical for both 2-D and 3-D configurations are not repeated in the 3-D column.

\begin{tabular}{lccc}
\hline Description & Symbol & \multicolumn{2}{c}{ Value } \\
\cline { 3 - 4 } & & 2 -D & 3 -D \\
\hline Domain size & $x,(y), h$ & $20 \times 40$ & $20 \times 20 \times 40$ \\
Ellipsoid axis length & $x,(y), h$ & $2 \times 8$ & $2 \times 2 \times 8$ \\
Reference porosity & $\phi_{0}$ & $10^{-2}$ & \\
Reference permeability & $k_{0}$ & 1 & \\
Fluid shear viscosity & $\mu^{f}$ & 1 & $(0,0,1)$ \\
Permeability power law exponent & $n_{k}$ & 3 & \\
Compaction bulk viscosity & $\eta_{\mathrm{c}}$ & 1 & $10^{-1}$ \\
Gravity acceleration & $g$ & 2 & 500 \\
Solid density & $\rho^{s}$ & 1 & \\
Fluid density & $\rho^{f}$ & $10^{-1}$ to 10 & \\
Rheological cst. (bulk to shear viscosity) & $C$ & 1 to $10^{3}$ & \\
Rheological cst. (compaction versus & $R$ & $10^{-2}$ & \\
decompaction) & & $\lambda_{p}$ & \\
Effective pressure transition zone & &
\end{tabular}

nodes that are located in the cell centres, like total pressure. Additional material properties, namely porosity and bulk viscosity, also reside in this location.

We utilize an iterative process to obtain a numerical solution that satisfies the non-linear discrete hydromechanical equations. We rely on an implicit solution procedure to progressively reduce the imbalance caused by the iterative pressures (fluid and total) and velocity fields. The iterative process is stopped once the hydromechanical equations are satisfied to a defined accuracy. We reformulate the hydromechanical eqs (1) and (2) as:

$$
\begin{aligned}
\nabla_{j}\left(\bar{\tau}_{i j}-\bar{p} \delta_{i j}\right)-\bar{\rho} g_{i} & =f_{\mathrm{v}_{i}}, \\
-\nabla_{k} v_{k}^{s}-\frac{\bar{p}-p^{f}}{\eta_{\phi}(1-\phi)} & =f_{\overline{\mathrm{p}}}, \\
-\nabla_{k}\left[\phi\left(v_{k}^{f}-v_{k}^{s}\right)\right]+\frac{\bar{p}-p^{f}}{\eta_{\phi}(1-\phi)} & =f_{\mathrm{p}^{\mathrm{f}}} .
\end{aligned}
$$

The right-hand side terms $\left(f_{\mathrm{v}_{i}}, f_{\overline{\mathrm{p}}}, f_{\mathrm{p}^{\mathrm{f}}}\right)$ are non-linear momentum, total continuity and fluid pressure residuals. They quantify the magnitude of imbalance of the hydromechanical equations. In this study, we focus primarily on the porosity wave regime as limit of two-phase flow. Since the matrix deformation experiences small strains only, we do not consider changes in the porosity field owing to advection (thus dropping the advective term from eq. 1). We approximate the porosity time derivative either by a backward Euler or a CrankNicolson $(\mathrm{CN})$ scheme and formulate it as:

$\frac{\partial \phi}{\partial t}=(1-\phi) \nabla_{k} v_{k}^{s}$.

We investigate two approaches to solve the coupled system of non-linear equations, thus minimize the magnitude the non-linear residuals; a direct-iterative (DI) solver workflow and a matrix-free pseudo-transient (PT) alternative. The DI method is based on extending the M2Di single-phase Stokes flow routines (Räss et al. 2017a) for two-phase flow. The Stokes equations are here coupled to a non-linear Darcy flow in order to compute the additional fluid pressure. To achieve this, we collect the coefficient matrix for the linearized operator and perform a direct-iterative solve. We rely on Newton linearization to converge the non-linearities in this DI procedure. In contrast, the alternative PT solving strategy relies on a matrix-free approach that does not require a matrix assembly for the linear operator. Thus, the memory requirements scale linearly with increasing problem size. Minimizing the residuals to reach the solution is performed iteratively using a numerical time-stepping (relaxation) strategy within a pseudo-time loop.

In a classical approach, solving the set of time-dependent nonlinear equations described above requires a five-step procedure (Fig. 3a) that includes: (1) an update of the non-linearly dependent parameters (listed in Section 2), (2) the evaluation of the nonlinear residual, (3) the assembly of the linear matrix operator, 4) a solve of the linearized system, (5) updating the solution and repeating (1) to (5) until convergence is reached. This procedure is then applied in a time-loop. We will follow such an approach in the direct-iterative solver, using a decoupled linear solving strategy for the linear system (4), and a Picard scheme for the non-linear iterations. Alternatively, the five-step procedure is rearranged in a single iteration loop that combines steps (3) to (5) in the pseudo-transient solver type (Fig. 3 b), thus simultaneously converging both the linear and the non-linear problem for each time-step. We will demonstrate that both PT and DI methods allow us to obtain an implicit solution of the two-way coupled system of equations in balance at physical time $(t+\Delta t)$.

We propose three different but complementary benchmark cases for validation of hydromechanical coupling in numerical codes: (1) a comparison of the DI against the PT results in 2-D, (2) a benchmark comparing numerical and exact analytical 1-D solutions of solitary waves and (3) a convergence study of 2-D numerical solutions with varying spatial and temporal resolutions. We provide additional technical information regarding the DI and the PT implementations within the Appendix A and the Appendix B, respectively. We report the DI versus PT comparison (1) within the Section 4.3, including a 2-D versus 3-D comparison. The two further benchmarks (2) and (3) are reported within the Appendix C. Readers interested in performance considerations of the various routines are directed to the Appendix D for further details. There we utilize the effective memory throughput as metric to evaluate how efficiently the memory-bounded PT routines perform in comparison to memory copy. We additionally time the convergence of one non-linear timestep of the 2-D PT and DI solvers using the wall-time metric. We finally report the parallel efficiency of the MPI-based mutli-GPU implementation of the 3-D PT solver. 

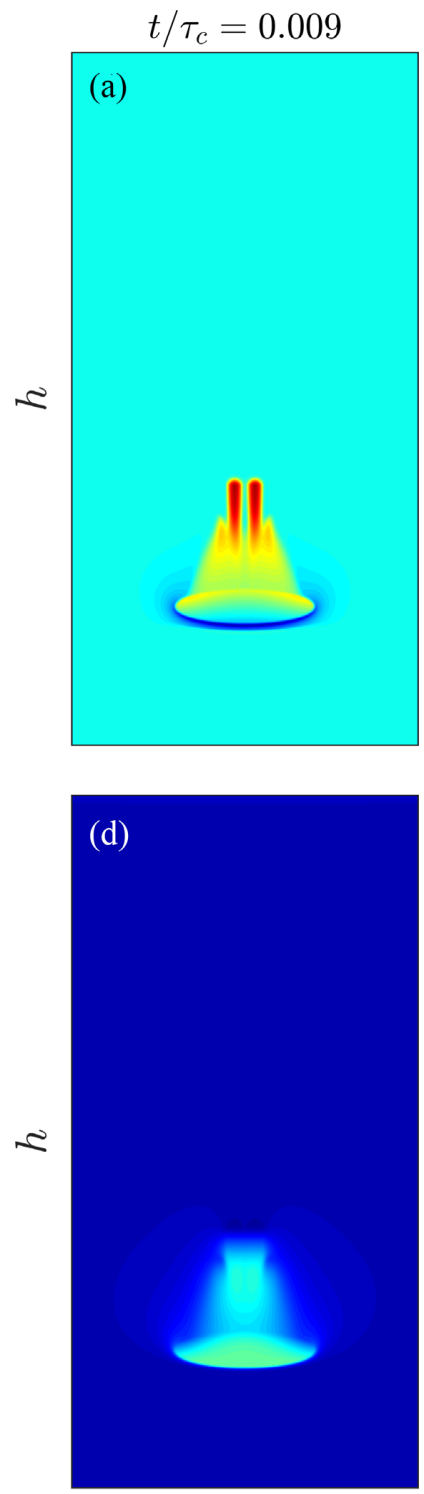

$x$
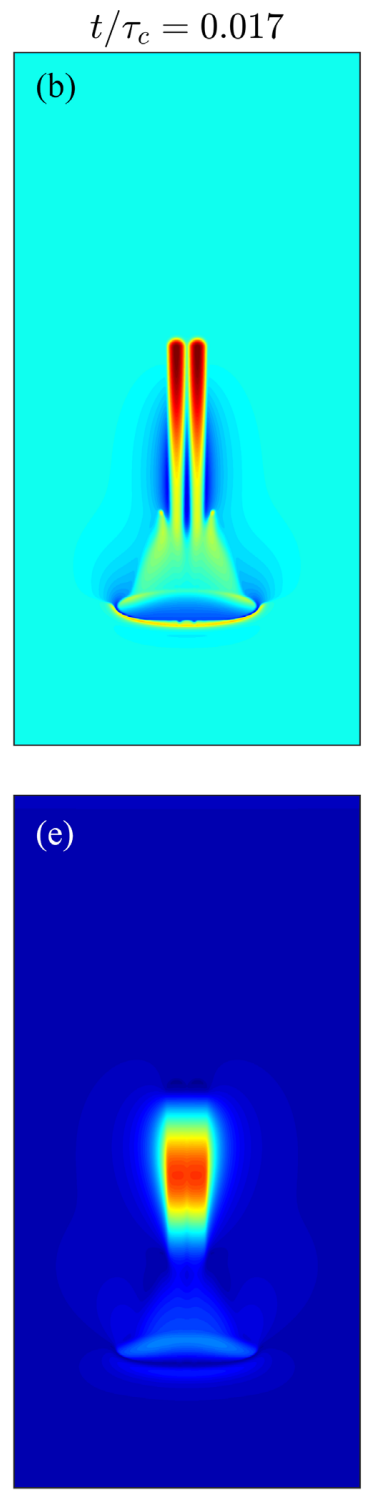

$x$
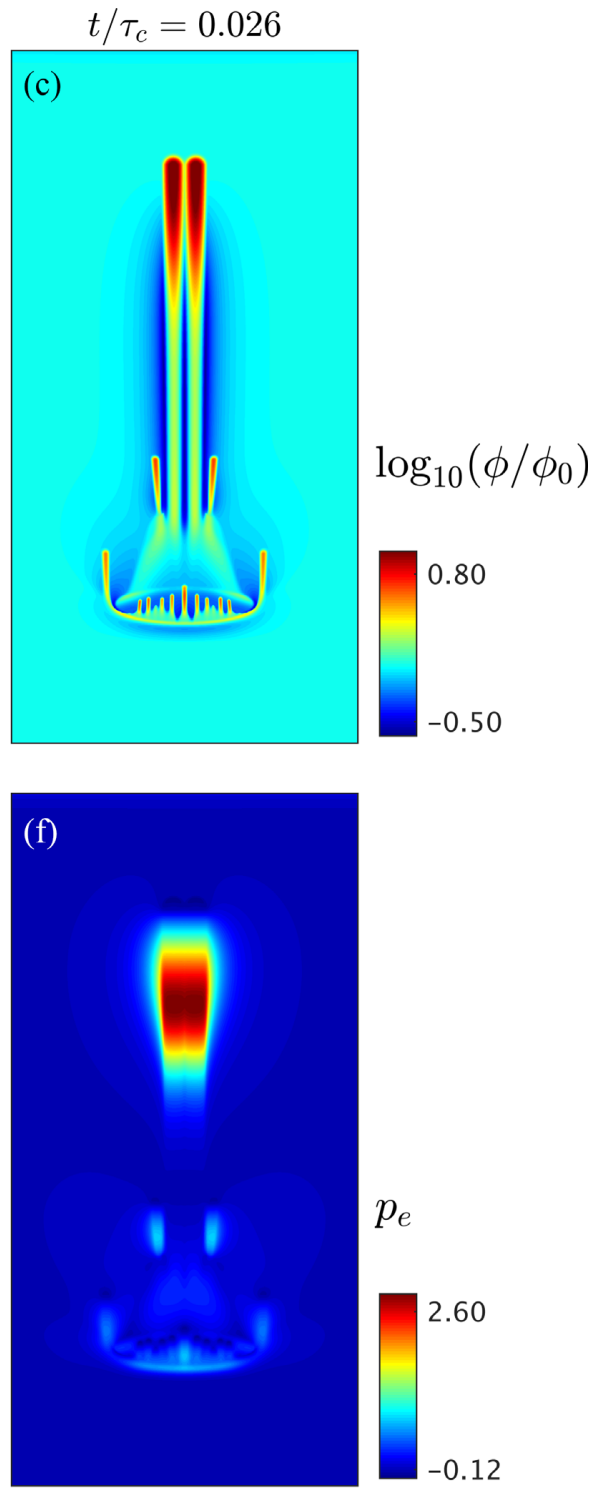

$x$

Figure 2. Evolution in a 2-D configuration of the formation and propagation of high-porosity channels for three snapshots in dimensionless time. (a) to (c) The $\log _{10}$ of the normalized porosity $\log _{10}\left(\phi / \phi_{0}\right)$ distribution. (d) to (f) The corresponding distribution of effective pressure $p_{e}$. The initial condition used for this simulation is the one presented in Fig. 1(b), and we used following parameters: $R=\eta_{\mathrm{c}} / \eta_{\mathrm{d}}=500, C=\mu_{s} /\left(\eta_{\mathrm{c}} \phi_{0}\right)=10^{-1}$. The simulation runs until the high-porosity channels reaches 85 per cent of the domain depth $(h)$. The grid resolution is of $2047 \times 4095$ gridpoints and a few days were needed to perform the close to 2000 time-steps on a Nvidia Titan Xp GPU. The related movie is available as supplementary material.

\subsection{The direct-iterative solver}

We use an analytical Newton scheme together with a DI method which permits us to obtain accurate non-linear solutions performing a limited number of non-linear iterations (see Appendix A1). The numerical solution, $\mathbf{x}=\left[\mathbf{v}, \overline{\mathbf{p}}, \mathbf{p}^{\mathbf{f}}\right]^{T}$, combining velocity $\mathbf{v}$, total pressure $\overline{\mathbf{p}}$ and fluid pressure $\mathbf{p}^{\mathbf{f}}$, are iteratively corrected with the update:

$\mathbf{x}^{k+1}=\mathbf{x}^{k}+\alpha \delta \mathbf{x}^{k+1}$

where the $\delta$ operator stands for the correction of $\mathbf{x}, \alpha$ is an optimization scalar and $k$ is the non-linear iteration index. The Newton corrections, $\delta \mathbf{x}=\left[\delta \mathbf{v}, \delta \overline{\mathbf{p}}, \delta \mathbf{p}^{\mathbf{f}}\right]^{T}$, are obtained by application of the inverse of the Jacobian matrix $\mathbf{J}_{\mathrm{HM}}$ to the current non-linear residual

$$
\mathbf{f}=\left[\mathbf{f}_{\mathrm{v}}, \mathbf{f}_{\overline{\mathrm{p}}}, \mathbf{f}_{\mathrm{p}^{\mathrm{f}}}\right]^{T}:
$$

$\delta \mathbf{x}^{k+1}=\left(\mathbf{J}_{\mathrm{HM}}{ }^{k}\right)^{-1} \mathbf{f}^{k}$.

We run a line search procedure to find the optimal optimization parameter $\alpha(0<\alpha \leq 1)$ satisfying:

$\min || \mathbf{f}\left(\mathbf{x}^{k}+\alpha \delta \mathbf{x}^{k+1}\right) \|_{\mathrm{L} 2}$

In the DI context, we have to formulate and assemble the Jacobian matrix that describes the gradients of the residuals with respect to the solutions:

$\mathbf{J}_{\mathrm{HM}}=\frac{\partial \mathbf{f}_{i}}{\partial \mathbf{x}_{j}}=\left[\begin{array}{ccc}\mathbf{J}_{\mathrm{vv}} & \mathbf{J}_{\mathrm{vp}} & \mathbf{0} \\ \mathbf{J}_{\mathrm{pv}} & \mathbf{J}_{\mathrm{pp}} & \mathbf{J}_{\mathrm{ppf}} \\ \mathbf{J}_{\mathrm{pfv}} & \mathbf{J}_{\mathrm{pfp}} & \mathbf{J}_{\mathrm{pf}}\end{array}\right]$. 
(a) Direct-Iterative method

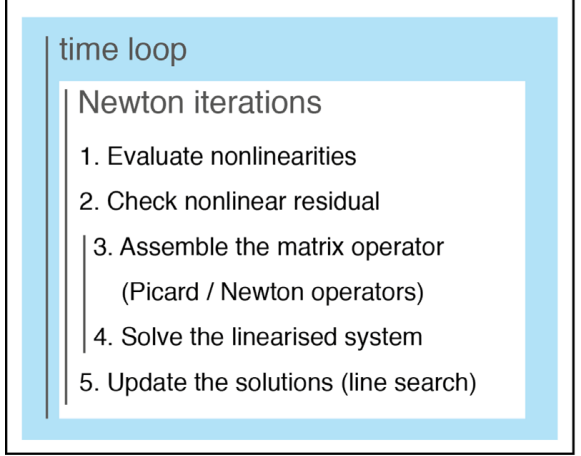

\section{(b) Pseudo-Transient method}

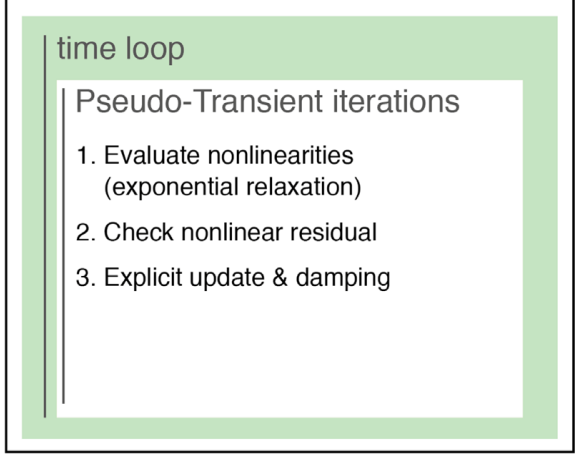

Figure 3. Algorithmic flowchart for both solving methods investigated in this study: (a) the direct-iterative solver (HM2Di code) and (b) the pseudo-transient solver (HM2Dpt code).

The $\mathbf{J}_{\mathrm{vv}}$ block represents the mechanical Jacobian matrix which adresses the porosity dependence of the density in the total force balance. The $\mathbf{J}_{\mathrm{pp}}$ block contains information about the sensitivity of the total pressure on the effective bulk viscosity. The $\mathbf{J}_{\mathrm{pf}}$ block is the Jacobian block associated with the fluid pressure Laplacian. $\mathbf{J}_{\mathrm{ppf}}$ and $\mathbf{J}_{\mathrm{pfp}}$ include coupling terms between $\bar{p}$ and $p^{f}$. The $\mathbf{J}_{\mathrm{pv}}$ and $\mathbf{J}_{\mathrm{pfv}}$ blocks respectively contain information about the sensitivity of $\bar{p}$ and $p^{f}$ with regard to the total velocity. The $\mathbf{J}_{\mathrm{vp}}$ block finally represent the gradient operator.

The Newton corrections for the hydromechanical system (eq. 10) are formulated as

$\delta \mathbf{x}=\left[\begin{array}{c}\delta \mathbf{v} \\ \delta \overline{\mathbf{p}} \\ \delta \mathbf{p}^{\mathbf{f}}\end{array}\right]=\left[\begin{array}{ccc}\mathbf{J}_{\mathrm{vv}} & \mathbf{J}_{\mathrm{vp}} & \mathbf{0} \\ \mathbf{J}_{\mathrm{pv}} & \mathbf{J}_{\mathrm{pp}} & \mathbf{J}_{\mathrm{ppf}} \\ \mathbf{J}_{\mathrm{pfv}} & \mathbf{J}_{\mathrm{pfp}} & \mathbf{J}_{\mathrm{pf}}\end{array}\right]^{-1}\left[\begin{array}{c}\mathbf{f}_{\mathrm{v}} \\ \mathbf{f}_{\overline{\mathrm{p}}} \\ \mathbf{f}_{\mathrm{p}_{\mathrm{f}}}\end{array}\right]$,

and are obtained via the DI method (see Appendix A2). We abort the non-linear iteration cycle once $\|\mathbf{f}\|_{\mathrm{L} 2}<$ tol $_{\text {nonlin }}^{\text {abs }}$ or $\|\mathbf{f}\|_{\mathrm{L} 2} /\|\mathbf{f}\|_{\mathrm{L} 2}^{\text {initial }}<$ tol $_{\text {nonlin }}^{\text {rel }}$ is satisfied. At this stage, the hydromechanical equations are satisfied to the desired accuracy. We summarize the main steps of this DI procedure in Fig. 3(a). We provide the MATLAB source code as part of the M2Di routines (Räss et al. 2017a) under the name HM2Di_Newton_GJI.m (https://bitbucket.org/lraess/m2di).

The employed DI method relies on the use of direct factorization methods (e.g. Cholesky), whose time-to-solution and memory requirements do not scale linearly with an increase in the number of degrees of freedom (DoF). Further, DI solvers may be complex to develop when the physical problem requires one to include several non-linearities and may not be an optimal solution approach when investigating 3-D configurations. However, the DI solution method is rather non-sensitive to large variations of material properties.

\subsection{The pseudo-transient solver}

To overcome the aforementioned limitations, we propose an alternative solving approach to the DI method, namely a pseudotransient or relaxation method. Resolving 3-D configurations on high spatial resolution including complex non-linearities are the main motivations to utilize pseudo-transient-based solvers, since they are capable of addressing limitations inherent to DI type of solvers. The relaxation method is a classical numerical technique to solve stationary (elliptic) problems (Frankel 1950). The method was extended in the 1960s to elastic problems (Otter et al. 1966) and more recently to elasto-plastic (Cundall 1982) and viscoelastic problems (Poliakov et al. 1993). The essence of the PT method consists to introduce physical transient terms into steady-state equations and integrating the equations forward in pseudo-time $\tau$. At this point, steady-state is attained, that is the pseudo-time derivatives vanish.

We show that the PT iterative solving strategy allows one to avoid an expensive assembly of a matrix for the linearized operator, resulting in a matrix-free approach. The combination of PT solvers with a matrix-free implementation of the finite-difference method shows by construction a linear scaling between memory usage and computed problem size (DoF). Such an approach is also well suited for parallel execution on modern hardware accelerators such as graphical processing units (GPUs), since the same instruction set is performed at every gridpoint in the numerical domain. The addition of message passing interface (MPI) protocols to the PT routines permits a non-expensive internal boundary values exchange by point-to-point MPI communication. Thus, a close-tolinear scaling of the routines on distributed-memory machines is expected.

Further, a PT-based iterative solver can be designed to simultaneously minimize both the linear and the non-linear problem in a single iteration loop. This is achieved by the relaxation of the non-linearities during the iterative procedure used to converge the linearized problem. Such an approach may significantly reduce the overall number of iterations needed to converge one physical time-step, once optimal relaxation parameters are found. We summarize the main steps of the PT method (Fig. 3b) and additional details regarding the PT continuation method with application to a generic example appear in the Appendix B. We provide the MATLAB source code under the name HM2Dpt_GJI.m (https://bitbucket.org/lraess/m2di).

\subsubsection{Application of the pseudo-transient continuation}

The system of non-linear coupled two-phase eqs (10) can thus be augmented with right-hand side time derivatives for momentum $\left(\rho \partial v_{i} / \partial t\right)$, mass $(\beta \partial \bar{p} / \partial t)$ and transient Darcian flow $\left(\partial p^{f} / \partial t\right)$. These terms represent acceleration (with $\rho$ as the inertial density), the elastic bulk rheology (with $\beta$ as the compressibility) and the transient fluid pressure diffusion, respectively. A solution to the incompressible two-phase flow problem requires all these transient terms to 
vanish. We express eq. (10) as:

$$
\begin{aligned}
& \nabla_{j}\left(\bar{\tau}_{i j}-\bar{p} \delta_{i j}\right)-\bar{\rho} g_{i}=\rho \frac{\partial v_{i}}{\partial \tau_{\mathrm{v}_{i}}}, \\
&-\nabla_{k} v_{k}^{s}-\frac{\bar{p}-p^{f}}{\eta_{\phi}(1-\phi)}=\beta \frac{\partial \bar{p}}{\partial \tau_{\overline{\mathrm{p}}}}, \\
&-\nabla_{k}\left[\phi\left(v_{k}^{f}-v_{k}^{s}\right)\right]+\frac{\bar{p}-p^{f}}{\eta_{\phi}(1-\phi)}=\frac{\partial p^{f}}{\partial \tau_{\mathrm{p}^{\mathrm{f}}}},
\end{aligned}
$$

where we substitute the physical time derivatives $\partial / \partial t$ with pseudotransient $(\mathrm{PT})$ or numerical ones $\partial / \partial \tau$. The introduced right-hand side PT terms are equivalent to residuals; one can thus iteratively reduce them as in the DI method.

The non-linear fields such as the effective permeability $k_{\phi} / \mu^{f}$ appearing in the Darcy flux (eq. 10) and the bulk viscosity $\eta_{\phi}$ (eq. 10) are evaluated at each pseudo-transient iteration $k$ using the current porosity, total pressure, fluid pressure solution fields. These quantities $\xi=\left[k_{\phi} / \mu^{f}, \eta_{\phi}\right]$ can be expressed as effective $\xi_{\text {eff }}$ and updated using the following relaxation:

$\xi_{\mathrm{eff}}^{k}=\exp \left[\left(1-\theta_{\xi}\right) \ln \left(\xi_{\mathrm{eff}}^{k-1}\right)+\theta_{\xi} \ln \left(\xi^{k}\right)\right]$,

where $\theta_{\xi}\left(0 \leq \theta_{\xi} \leq 1\right)$ is a relaxation factor. This continuation method progressively relaxes the effective quantities towards the physical ones $\left(\xi_{\text {eff }} \rightarrow \xi\right.$ ) during the PT iterations.

The momentum, total pressure and fluid pressure evolution equations necessitate appropriate pseudo-time steps, $\Delta \tau_{v_{i}}, \Delta \tau_{\overline{\mathrm{p}}}$ and $\Delta \tau_{\mathrm{p}^{\mathrm{f}}}$. As we seek for the steady-state (i.e. the pseudo-time derivatives vanish) one can freely define the pseudo-time steps in order to achieve the fastest convergence rates:

$$
\begin{aligned}
\Delta \tau_{\mathrm{v}_{i}} & =\frac{\min \left(\Delta x_{i}\right)^{2}}{2.1 n_{\operatorname{dim}} \mu_{s}\left(1+\eta_{\mathrm{b}}\right)}, \\
\Delta \tau_{\overline{\mathrm{p}}} & =\frac{2.1 n_{\operatorname{dim}} \mu_{s}\left(1+\eta_{\mathrm{b}}\right)}{\max \left(n_{i}\right)}, \\
\Delta \tau_{\mathrm{p}^{\mathrm{f}}} & =\frac{\min \left(\Delta x_{i}\right)^{2}}{2.1 n_{\text {dim }} k_{\phi} / \mu^{f}},
\end{aligned}
$$

where $\mu_{s}$ stands for the scalar shear viscosity, $\eta_{\mathrm{b}}$ is a scalar and constant numerical bulk viscosity analogy, $n_{\text {dim }}$ stands for the number of spatial dimensions, $\Delta x_{i}$ and $n_{i}$ are the grid step sizes and number of gridpoints in the $i$ direction ( $i=x$ in 1-D, $x, y$ in 2-D and $x, y, z$ in 3-D), respectively. In order to reduce the number of parameter, we assume from here that $\rho$ and $\beta$ are equal to 1.0. $\Delta \tau_{v_{i}}$ is the pseudo-time step used to integrate the momentum equation. It is constructed by multiplying the explicit Courant-Friedrichs-Lewy condition (CFL) for viscous flow time $\operatorname{step} \min \left(\Delta x_{i}\right)^{2} /\left(2.1 n_{\operatorname{dim}} \mu_{s}\right)$ by $1 /\left(1+\eta_{\mathrm{b}}\right)$, which includes a numerical analogy of the bulk viscosity $\eta_{\mathrm{b}}$. We use the denominator of $\Delta \tau_{\mathrm{v}_{i}}$ as numerator in the definition of $\Delta \tau_{\overline{\mathrm{p}}}$ leading to an empirically defined pseudo-time step for the continuity equation. Thus, the iteration strategy is less sensitive ot the physical shear viscosity $\mu_{s}$ which allows for optimal convergence of the mechanical problem. $\Delta \tau_{p^{f}}$ stands for the explicit fluid pressure diffusion pseudo-time step satisfying the CFL condition. All three time step definition may be in addition multiplied by a reduction factor $(\leq 1.0)$, mainly due to the presence of significant non-linearities.

Using a dimensional analysis, we confirm that the product of $\rho^{-1} \Delta \tau_{\mathrm{v}_{\mathrm{i}}}\left[\mathrm{m}^{2} \mathrm{~Pa}^{-1} \mathrm{~m}^{-1}\right]$ by the momentum balance terms $\left[\mathrm{Pa} \mathrm{m}{ }^{-1}\right]$ permits to retrieve increments of solid velocity $\left[\mathrm{m} \mathrm{s}^{-1}\right]$. We apply similar reasoning to the total continuity equation, where $\beta^{-1} \Delta \tau_{\overline{\mathrm{p}}}$ [Pa.s] multiplies $\nabla_{k} v_{k}^{s}\left[\mathrm{~s}^{-1}\right]$ to produce dynamic pressure increments in $[\mathrm{Pa}]$. We additionally highlight that $k_{\phi} / \mu^{f}$ refers to entire field (defined for every gridpoint) and so the application of $\Delta \tau_{\mathrm{p}^{\mathrm{f}}}$ is analogous to rely on a diagonal preconditioner in matrix-based solvers.

The velocity, total pressure and fluid pressure fields are updated at each PT iteration using current values of pseudo-time steps and residuals:

$$
\begin{gathered}
v_{i}^{k}=v_{i}^{k-1}+\Delta \tau_{\mathrm{v}_{i}} f_{\mathrm{v}_{i}}^{k}, \\
\bar{p}^{k}=\bar{p}^{k-1}+\Delta \tau_{\overline{\mathrm{p}}} f_{\overline{\mathrm{p}}}^{k}, \\
p^{f^{k}}=p^{f^{k-1}+\Delta \tau_{\mathrm{p}^{\mathrm{f}}} f_{\mathrm{p}^{\mathrm{f}}}^{k} .}
\end{gathered}
$$

The PT iterations are performed as for the DI method, until one of the following criteria $\|\mathbf{f}\|_{\mathrm{L} 2}<$ tol $_{\text {nonlin }}^{\text {abs }}$ or $\|\mathbf{f}\|_{\mathrm{L} 2} /\|\mathbf{f}\|_{\mathrm{L} 2}^{\text {initial }}<$ tol $_{\text {nonlin }}^{\text {rel }}$.

\subsubsection{Accelerating the convergence}

Unfortunately, in 1-D, the most straightforward update algorithm (eq. 20) based on a first order scheme $(\partial / \partial \tau)$ requires order of $n^{2}$ iterations to converge to the stationary solution, where $n$ is the total number of unknowns or gridpoints. Interestingly, a second order scheme $\left(\alpha \partial^{2} / \partial \tau^{2}+\partial / \partial \tau\right)$ permits to reach enhanced convergence rates when selecting the appropriate damping parameter $\alpha$ (Frankel 1950). Damping acts like successive over-relaxation within the pseudo-time iterations loop (Yang \& Mittal 2014). Adding to the current residual $\left(f^{k}\right)$ a significant fraction of the previous update $\left(g^{k-1}\right)$ strongly impacts the overall convergence rate. We apply damping or over-relaxation on both the momentum and the fluid pressure equations (eq. 20):

$$
\begin{gathered}
v_{i}^{k}=v_{i}^{k-1}+\Delta \tau_{\mathrm{v}_{\mathrm{i}}} g_{\mathrm{v}_{i}}^{k}, \\
p^{f^{k}}=p^{f^{k-1}+\Delta \tau_{\mathrm{p}^{\mathrm{f}}} g_{\mathrm{p}^{\mathrm{f}}}^{k},}
\end{gathered}
$$

reformulating both velocity and fluid pressure residuals as updates $g$ :

$g_{\mathrm{v}_{i}}^{k}=f_{\mathrm{v}_{i}}^{k}+\left(1-\frac{v_{\mathrm{v}}}{n_{i}}\right) g_{\mathrm{v}_{i}}^{k-1}$,

$g_{\mathrm{p}^{\mathrm{f}}}^{k}=f_{\mathrm{p}^{\mathrm{f}}}^{k}+\quad v_{\mathrm{p}^{\mathrm{f}}} g_{\mathrm{p}^{\mathrm{f}}}^{k-1}$,

where $\alpha$ can be expanded to $\left(1-v_{\mathrm{v}} / n_{i}\right)$ in the velocity update $g_{\mathrm{v}_{i}}^{k}$ and to $v_{\mathrm{p}^{\mathrm{f}}}$ in the fluid pressure update $g_{\mathrm{p}}^{k}$. Optimal values of $v_{\mathrm{v}}$ reside within the range $\left(1 \leq v_{\mathrm{v}} \leq 10\right)$, where $n_{i}$ is the spatial grid resolution in the direction $i$. Values of $v_{\mathrm{p}^{\mathrm{f}}}$ vary between 0.7 and 0.92 depending on $n_{i}$. See Tables (B1) and (B2) in the Appendix B for further information. Such damping or over-relaxation strategy significantly accelerate the residuals convergence rates (Cundall 1982; 1987; Poliakov et al. 1993). Notably, the elastic analogy of this approach (Cundall \& Strack 1979) is successfully used in the FLAC geotechnical software (Cundall et al. 1993). The described implementation refers to the code (HM2Dpt) appended to this work as supplementary material.

\subsubsection{Parallel implementation on GPUs}

One underlying reason to explore iterative and matrix-free solving approaches is such methods' ability to deliver the best performance in parallel implementation. Since no global matrix needs to be assembled at any point in the solving procedure, all operations are identical on each gridpoint in the computational domain. Finitedifference derivatives are constructed by stencil operation, where only neighbouring values accesses are mandatory. We successfully implemented the PT solver to perform the computation on GPUs, 
using the CUDA extension to C language. GPUs are many-core processors, initially designed to refresh screen pixels at high framerates. In the CUDA framework, each small GPU core can concurrently execute several threads. Thus, we assign to each thread one single cell of the computational domain, making it possible to perform in parallel the same operation simultaneously on the entire domain. A careful implementation of synchronization barriers avoids race conditions and prevents reading from an array that is not yet fully updated.

Further parallelism can be achieved by porting the single-GPUbased routines to run on several GPUs simultaneously, using the message-passing interface (MPI) library. Thus, we enable the routines to be executed on distributed-memory machines, multipleGPU workstations and the largest GPU-based supercomputers. Such an approach is particularly relevant for high resolution 3-D computations involving more than 1 billion gridpoints (e.g. Omlin et al. 2017b; Räss et al. 2018). Further performance considerations are reported within the Appendix D.

\subsection{Comparison of the DI and PT solvers}

To validate the two solving strategies, we propose a set of benchmark simulations. We aim to evaluate the solution's sensitivity on both the non-linear residual threshold and the spatial resolution. The results we report in this section use the configuration described in Section 3 as initial conditions. The simulation runs until the highporosity channel, also called porosity wave, reaches 85 per cent of the domain depth. In this study, we report results for the PT solver using single-precision arithmetic performed on a Nvidia GTX Titan $\mathrm{Xp}$ (Pascal) GPU. The DI solver is an extension to the M2Di routines and is implemented in MATLAB, running on an Intel i5 processor using double-precision arithmetic.

\subsubsection{The influences of non-linear iterations}

The influences of the non-linear threshold are more pronounced in the PT than in the DI approach (Fig. 4). Both the linear and the non-linear residuals are considered in the PT approach, while in the DI solving procedure, each non-linear step encounters an accurate solve of the linearized problem. The reported end-members of tested absolute non-linear tolerance ( tol $_{\text {nonlin }}$ ) start at tol $\mathrm{l}_{\text {nonlin }}=10^{-3}$ and spread over five orders of magnitude for the DI solver, and over two for the PT solver. Since the PT simulations are performed on a GPU using single-precision arithmetic, the lowest achievable value is tol nonlin $=10^{-5}$ (Fig. 4c). The double-precision arithmetic used in the MATLAB framework for the DI solve allows the absolute non-linear tolerance tol $1_{\text {nonlin }}=10^{-8}$ (Fig. 4f). At values of tol ${ }_{\text {nonlin }}$ $=10^{-3}$, both PT and DI solvers show deviations from converged solutions, even though the DI solver shows less dramatic deviation than the PT solver (Figs 4b and e, respectively). The line plots show the evolution over dimensionless time of the maximum normalized porosity values (Fig. 4a) and for the vertical location of the maximal porosity region (Fig. 4d). The PT results (continuous lines)

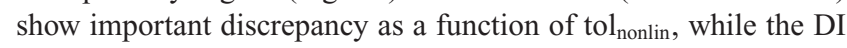
results (square and triangular markers) are less affected. The major outcome of this sensitivity analysis reports single-precision (tol nonlin $_{\text {n }}$ $=10^{-5}$ ) PT results that match the best-converged double-precision DI $\left(\right.$ tol $\left._{\text {nonlin }}=10^{-8}\right)$ results. Discrepancy in the non-converged runs ( tol $_{\text {nonlin }}=10^{-3}$ ) arises from the fact that the DI solver type relies on a converged linearized problem for each non-linear step. In contrast, both linear and non-linear residuals are converged simultaneously in the PT workflow, resulting in different realizations for non-converged simulations.

\subsubsection{The influence of spatial resolution}

The continuous increase in computing power allows high spatial resolution simulations to become feasible within reasonable execution times. Investigating the impacts of resolution increase on a given problem allows us to assess whether such high resolution is required and allows us to find the optimal resolution, leading to a physically converged solution. Here, we report the influences of variations in grid resolution for two-phase flow using both PT and DI solver types. The three tested resolutions show different results, but tend to converge towards a stable solution while increasing the number of gridpoints (Figs 5). Both the PT (Figs 5b-e) and DI (Figs 5g-i) runs clearly confirm that high spatial resolution is mandatory in order to accurately converge towards a stable solution. The line plots show the evolution over dimensionless time of the maximum values of normalized porosity (Fig. 5a) and the vertical location of the maximal porosity region (Fig. 5f). In contrast to the non-linear tolerance benchmark, the spatial resolution test shows identical behaviour for both DI and PT solving approaches; thus, the markers (DI solver) are located on top of the lines (PT solver) for all tested resolutions (Figs 5a and $\mathrm{f}$ ).

We confirm the convergence of the numerical results towards a stable two-channels morphology by providing a very high resolution simulation involving $2048 \times 4096$ gridpoints (Fig. 5e). While the two-channel morphology survives, some second order features differ between the two highest reported grid resolutions (Figs $5 \mathrm{~d}$ and e), such as the secondary channels sourcing at the lower bound of the initial elliptic porosity perturbation. The very high resolution run (Figs 5e) features four times more gridpoints within both the horizontal and vertical directions (in comparison to runs from Figs $5 \mathrm{~d}$ and i), increasing by the same factor 4 the number of gridpoints per compaction length, the latter governing the channel width. The highest reported numerical grid resolution (Figs 5e) cannot be achieved using the DI approach, due to the non available RAM requirements.

\subsubsection{A comparison of 2-D and 3-D runs}

We extended the two-phase flow-solver to compute the coupled physics in three spatial dimensions. Thus, the resulting 3-D routines are based on the PT matrix-free implementation and combine CUDA $\mathrm{C}$ to MPI running in parallel on GPU-based distributedmemory supercomputers. The resulting final stages are reported (Fig. 6) for three different resolutions for both the 2-D and 3-D comparative resolutions. The $3-\mathrm{D}$ configuration is identical to the 2-D initial configuration, with an additional $y$ dimension being equivalent to the $x$ dimension (as described in Fig. 1). The 2-D ellipse is converted to a 3-D ellipsoid, with an identical major/minor axis ratio. A cross-section in the middle of the $y$-axis $\left(\right.$ at $\left.\mathrm{L}_{y} / 2\right)$ is extracted from the 3-D cube in order to be compared to the 2-D configuration. The results clearly show a significant yet systematic discrepancy between the 2-D and the 3-D simulations. While the 2-D simulation results (Figs 6b-d) tend towards a stable solution that shows two thin distinct channels, the 3-D analogous runs (Figs $6 \mathrm{f}-\mathrm{h}$ ) organize highporosity regions into one single and larger channel. This systematic difference in shape mainly result from the 3-D nature of the investigated process, as discussed in Omlin et al. (2017b). In contrast to other strain localization mechanisms such as viscous shear-heating 

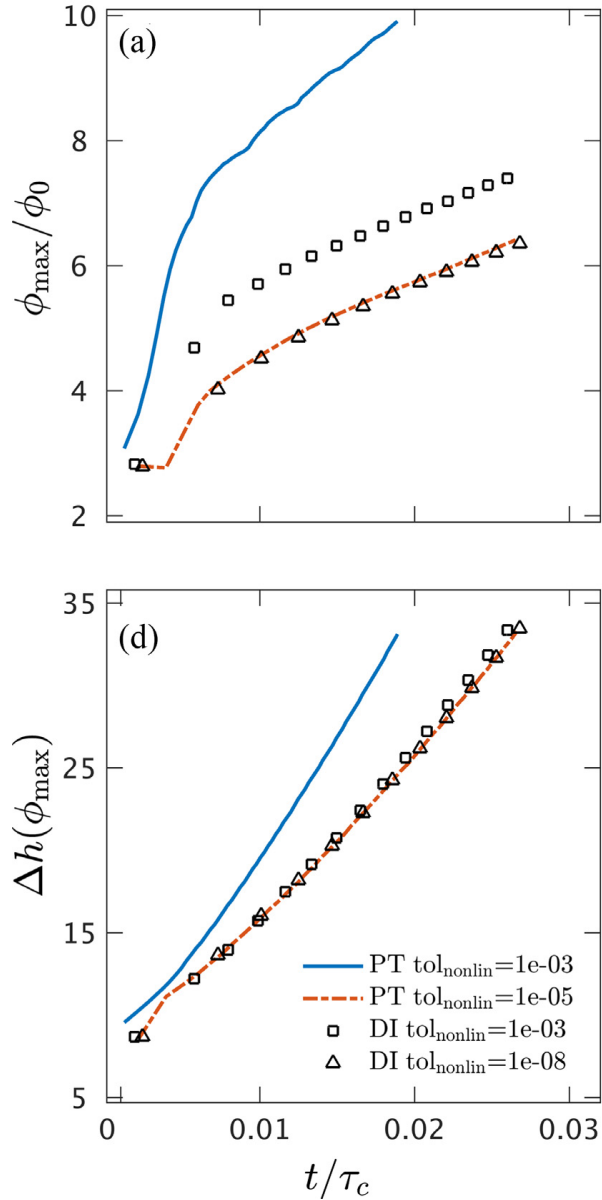
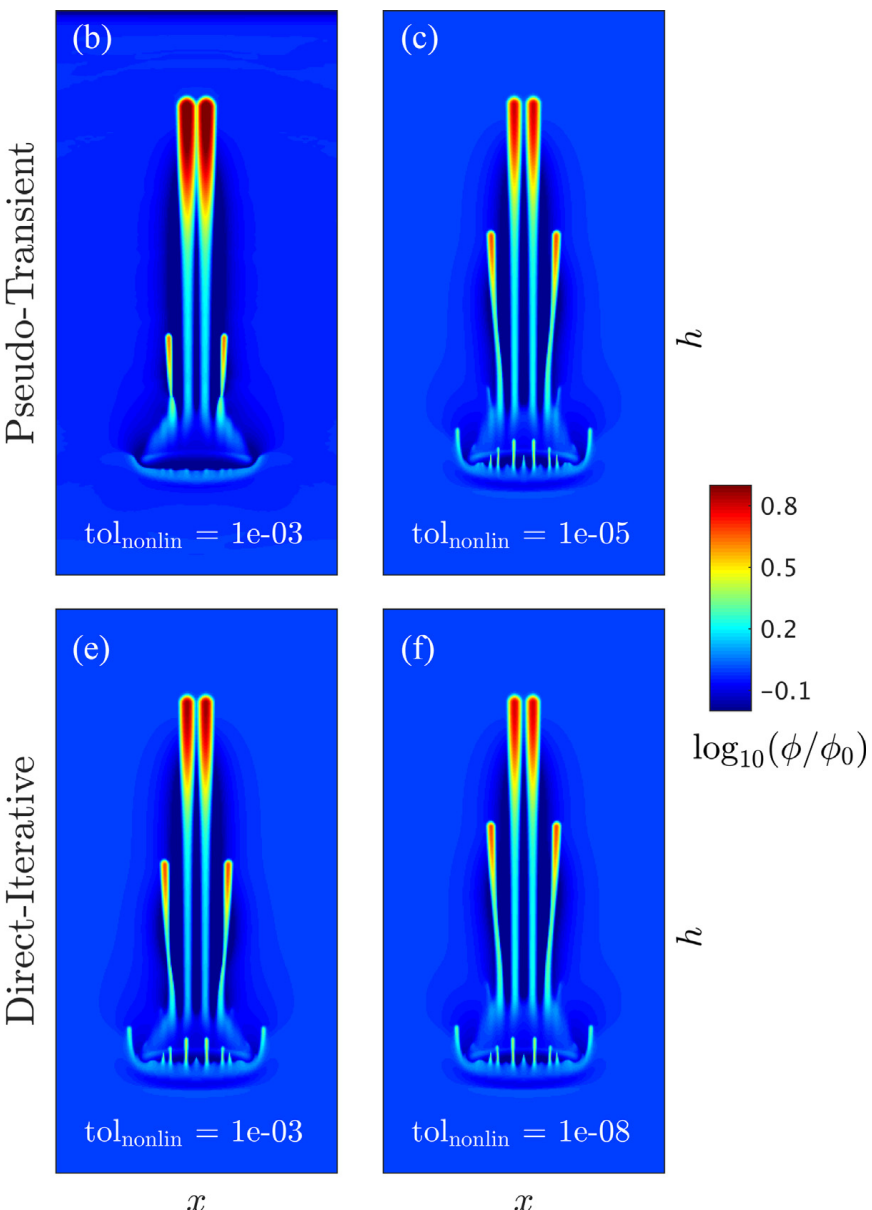

$x$

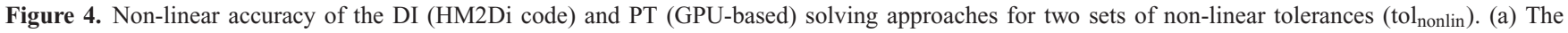
normalized maximal values of porosity $\left(\phi_{\max } / \phi_{0}\right)$ and (d) the vertical distance from initial perturbation $\left(\Delta h\left(\phi_{\max }\right)\right)$, both plotted against dimensionless time $\left(t / \tau_{c}\right)$. The lines represent the PT solver and the markers the DI solver. The corresponding 2-D final steps are reported for the PT solver for the poorly (b) and accurately (c) converged results, and by analogy (e) and (f) for the DI method, respectively. Since the PT implementation is computed on GPUs using single-precision arithmetic, the lowest achievable non-linear tolerance is tol ${ }_{\text {nonlin }}=10^{-5}$. The colour axis represents the $\log _{10}$ of the normalized porosity $\left(\phi / \phi_{0}\right)$.

or folding and necking, porosity waves cannot be assumed infinite in the third (out-of-plane) dimension when computed in 2-D. The resulting drainage area and available fluid fluxes in 3-D are much more important. Thus, the increased availability of fluid already in the initial condition impacts the overall dynamic. The results' dependence on the number of spatial dimensions must be carefully assessed, since implications in terms of transported volume of fluids and propagation speeds are not negligible.

\section{RESULTS}

\subsection{Hydromechanical fluid focussing in 2-D: a systematic study}

We performed a systematic investigation to understand the parameters that influence key properties of the two-phase flow channels, such as their propagation velocity and their shape. We performed more than 400 high-resolution forward 2-D runs, varying the bulk viscosity decompaction over compaction ratio $\eta_{\mathrm{d}} / \eta_{\mathrm{c}}$ and the bulk to shear viscosity ratio $\mu_{s} /\left(\eta_{c} \phi_{0}\right)$. For each simulation, the computational domain size was discretized in $512 \times 1024$ gridpoints, and the simulation lasted until the channel reached 85 per cent of the domain depth. We performed single-precision computations on a Nvidia Titan Xp GPU using single-precision arithmetic. The achieved nonlinear convergence threshold was $10^{-5}$ for every run. We realized the entire systematic sequentially on a single GPU, which took about one week for the reported resolution. For each simulation, we monitored the maximal velocity of the upward-propagating porosity wave $\max \left(v_{\text {wave }}\right)$, the maximal Darcy flux normalized over background Darcy velocity $\max \left(v_{y}^{\text {Darcy }}\right) / v_{\mathrm{BG}}^{\text {Darcy }}$, and both the minimal and maximal solid velocities $\min \left(v_{y}^{\mathrm{s}}\right) / v_{\mathrm{BG}}^{\text {Darcy }}$ and $\max \left(v_{y}^{\mathrm{s}}\right) / v_{\mathrm{BG}}^{\text {Darcy }}$, respectively (Fig. 7). The wave velocity $v_{\text {wave }}$ stands for the ratio of vertical displacement of the maximal value of porosity within the channel over time. The background Darcy velocity is the one defined for the background porosity fraction $\phi_{0}$. The porosity wave propagation velocity reflects the time needed to propagate the tip of the high-porosity channel over a given vertical distance. The 2-D systematic maps report an increasing wave velocity, while decreasing both the decompaction over compaction bulk viscosity ratio 

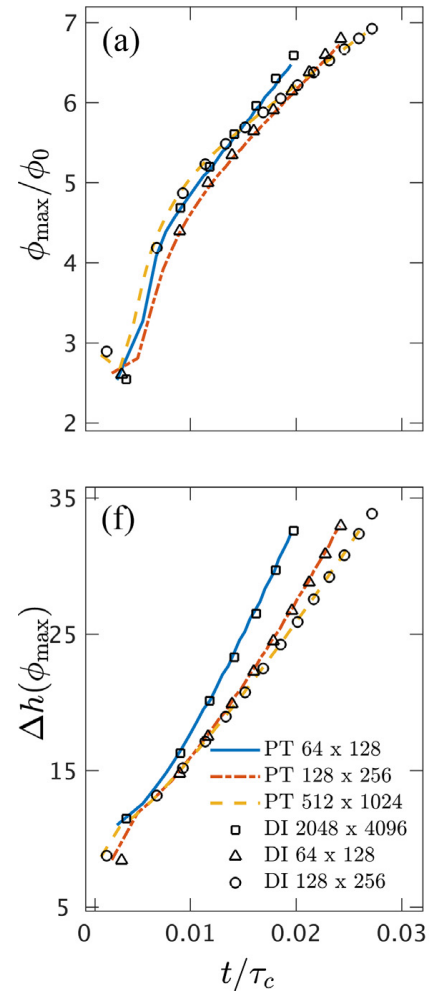
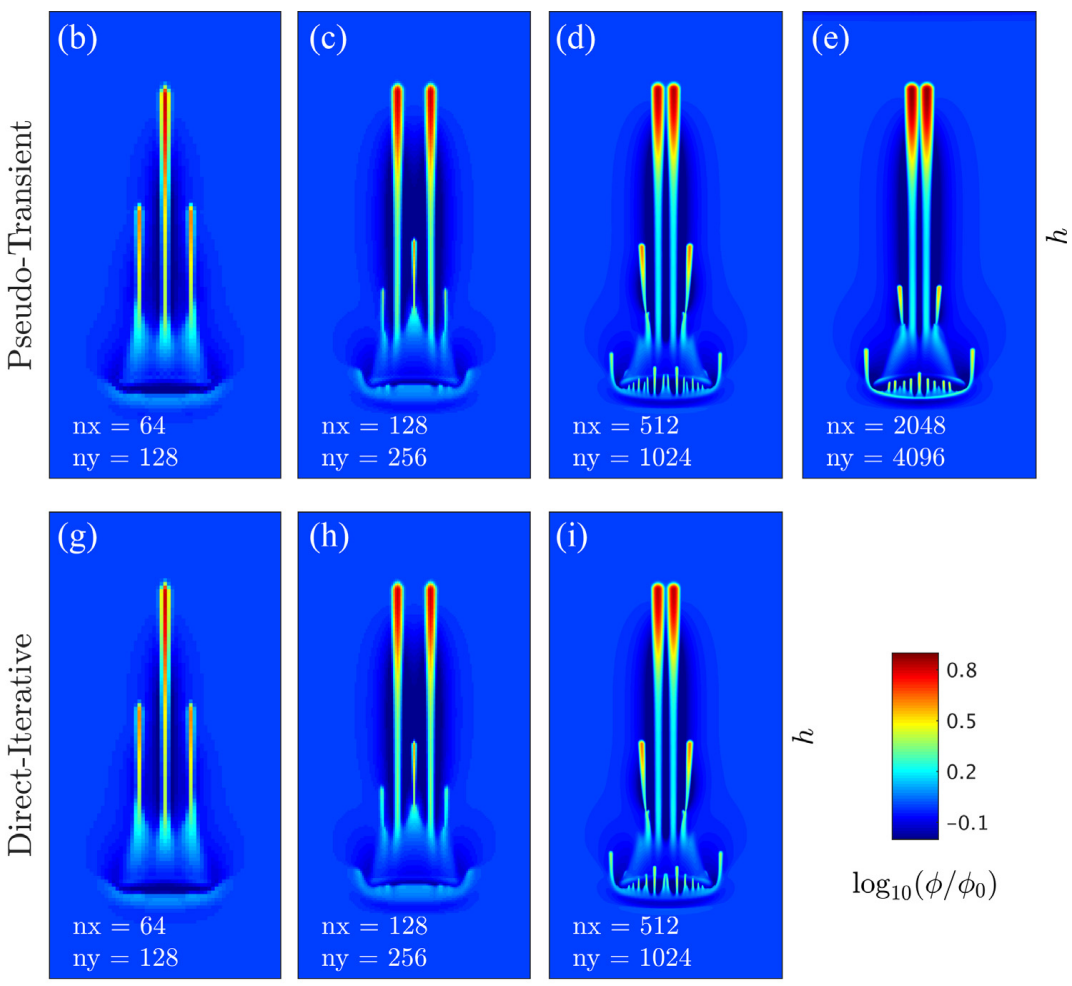

$x$ $\eta_{\mathrm{d}} / \eta_{\mathrm{c}}$ and the bulk to shear viscosity ratio $\mu_{s} /\left(\eta_{\mathrm{c}} \phi_{0}\right)$. The normalized Darcy flux partly follows a similar trend, while both the solid minimum and maximum velocities are mainly influenced by the bulk to shear viscosity ratio.

\subsection{Selected end-member runs}

Four end-member simulations of this systematic study are highlighted, providing insights into the 2-D spatial distribution of normalized porosity (Fig. 8), the vertical fluid flux (Fig. 9) and the non-linear bulk viscosity (Fig. 10). We report the 2-D field and plot the last time-step for every simulation, where the location and the symbols correspond to those on the systematic maps (Fig. 7). The selected runs show important variations in the reported endmember combination of parameters, leading to significant changes in propagation regimes. For decompaction over compaction bulk viscosity ratios and bulk to shear viscosity ratios close to 1 , the initial high-porosity anomaly propagates upwards in a blob-shaped soliton (circle symbol). The opposite end-member combination of parameters (star symbol) triggers the formation of sharply defined high-porosity channels (Fig. 8) with locally high fluid fluxes (Fig. 9) and extremely localized low values of non-linear bulk viscosity (Fig. 10). Note that the colour axis is different for every subplot and is therefore reported, since the important changes in regimes induce large variations in the parameter range and may saturate some plots.

\subsection{Data-collapse}

Finally, we explain the maximal wave velocity values of the entire systematic study as a function of the combined decompaction over compaction bulk viscosity $\eta_{\mathrm{d}} / \eta_{\mathrm{c}}$ and bulk to shear viscosity ratios $\mu_{s} /\left(\eta_{\mathrm{c}} \phi_{0}\right)$. A naive linear combination of the two parameters shows diverging trends and does not explain the trend of the maximal wave velocities (Fig. 11a). A more elaborate combination of the two investigated parameters, as reported in the $x$-label (Fig. 11b), shows the relative importance of $\eta_{\mathrm{d}} / \eta_{\mathrm{c}}$ and $\mu_{s} /\left(\eta_{\mathrm{c}} \phi_{0}\right)$ on explaining the increasing wave velocity $\max \left(v_{\text {wave }}\right)$. The reported data can be cast into three major regimes. Most runs exhibit a single-channellike mode, tending towards more pronounced focussing and faster propagation rates with an increase in the problem's non-linearity. At some critical point, the single-channel modes transform into a two-channel-like motion. The impact of this macroscopically different behaviour is the shift in the reported maximal velocity values from the displayed fitting curve $y=370 x^{0.22}$. This major change in propagation regime reflects the underlying non-linearities of the investigated coupled physics. With an increasing viscosity ratio, the effective compaction length (or characteristic length scale) tends to reduce, developing narrower channels. The observed shift in maximal wave velocities is also reflected in the maximal values of fluid fluxes (Fig. 7).

The fitting formula $1 /\left[\eta_{\mathrm{d}} / \eta_{\mathrm{c}}+0.025 \eta_{\mathrm{c}} \phi_{0} / \mu_{s}\right]$ suggests that the the maximal wave velocity is mainly function of the $\eta_{\mathrm{d}} / \eta_{\mathrm{c}}$ ratio - the decompaction weakening - while only a small fraction 

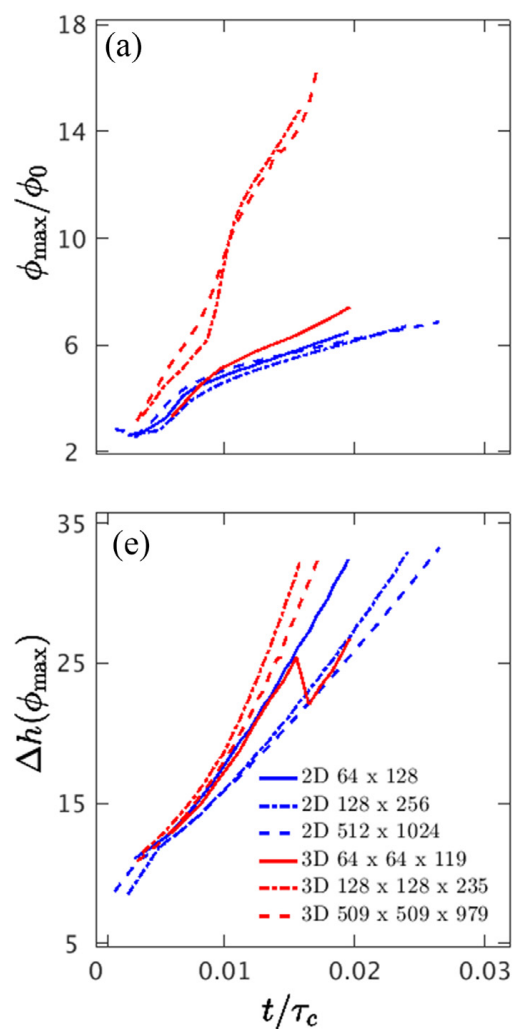
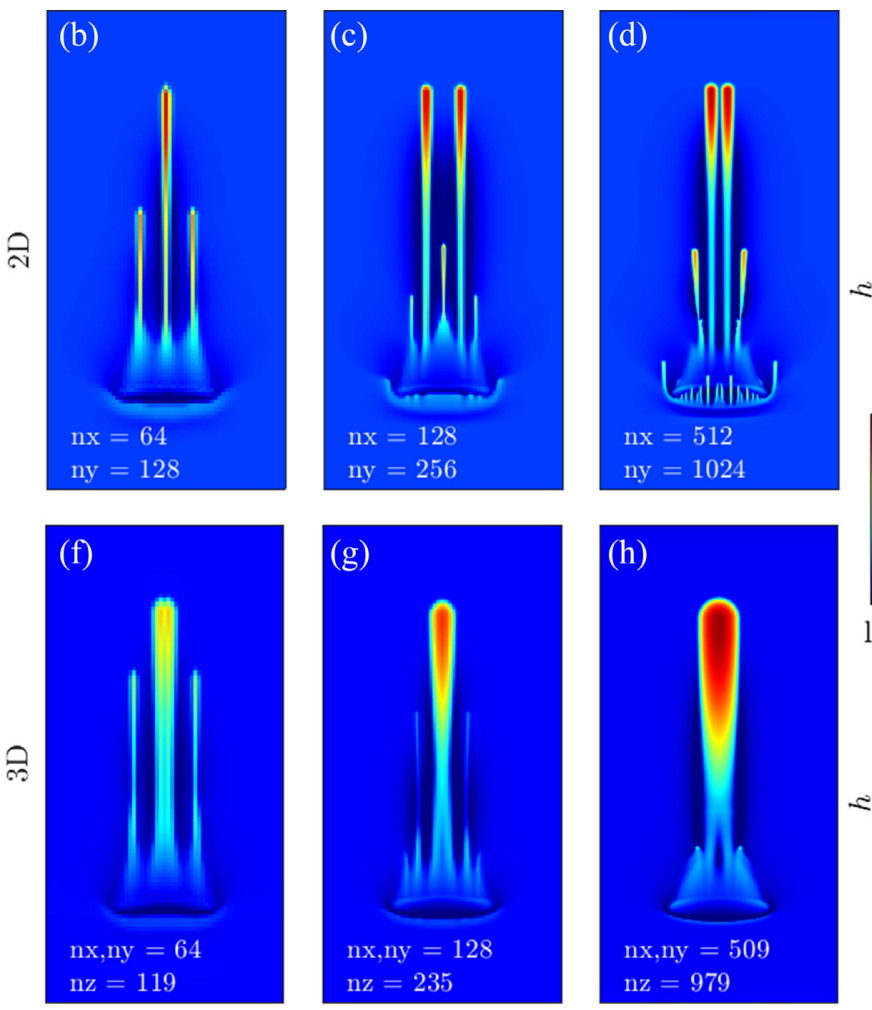

$x$ is sensitive to the bulk to shear viscosity ratio. These results suggest that volumetric deformation and tensile failure of the considered environments may primary dictate the propagation dynamic of the channels. Moreover, the maximal wave ascent velocity augments in a non-linear fashion together with an increase in decompaction weakening. This behaviour reflects that regimes where high porosity channels propagate at elevated speed exhibit a significant asymmetry in compaction over decompaction of the porous medium.

\subsection{Extension to power law shear viscosity in 3-D}

The two-phase flow model we rely on includes three important rheological parameters to quantify the bulk and shear viscous creep behaviour of the porous medium and the pore connectivity, namely $\eta_{\phi}, \mu_{s}$ and $k_{\phi}$. We systematically investigated the non-linear dependance of $\eta_{\phi}$ on $p_{e}$ and utilized a Carman-Kozeny power-law relation to define $k_{\phi}$. We assumed the linear solid shear viscosity $\mu_{s}$ to remain constant in space and time (linear); this permitted us to discriminate between several potential non-linear feedbacks ultimately impacting the evolution of the porosity distribution.

However, viscous shear deformation of rocks may actually be sensitive to variations in strain-rate and temperature. The rather shallow environments our study targets may be considered as isothermal due to their shallow location justifying not considering thermal effects at this stage. However, strain rate dependance of shear viscosity will have to be considered as important non-linear feedback within shallow environments such as sedimentary basins. We here provide a first 3-D simulation at very high numerical resolution (2 billion gridpoints) including a power law strain rate dependant solid shear viscosity $\mu_{s}$ expressed as:

$\mu_{s}=\frac{1}{\dot{\epsilon}_{I I}^{\frac{n-1}{n}}+\frac{1}{\mu_{0}}}$,

where $n=3$ is the power law exponent, $\mu_{0}$ is the reference viscosity for negligible strain rates and where $\dot{\epsilon}_{I I}=\sqrt{1 / 2 \dot{\epsilon}_{i j} \dot{\epsilon}_{i j}}$ is the square root of the second invariant of the deviatoric strain rate tensor. We rely on the continuation method introduced for $k_{\phi} / \mu_{f}$ and $\eta_{\phi}$ (eq. 18) in order to progressively relax the effective nonlinear shear viscosity towards the physical one $\mu_{s}$ during the PT iterations.

Investigating the first order features confirms the sustained occurrence of the high-porosity channels when considering a strain-rate dependent shear rheology. The upward-propagating porosity waves triggers highly localized matrix strain rates, which tend to locally lower the background shear viscosity by about one order of magnitude (Fig. 12a); this provides a physical mechanism to locally reduce the values of the $\mu_{s} /\left(\eta_{\mathrm{c}} \phi_{0}\right)$ parameter investigated in the systematic study. The non-linear bulk viscosity $\eta_{\phi}$ is sensitive to both the porosity averaged solid shear viscosity $\mu_{s}$ and the effective pressure $p_{e}$. In the present scenario, $\mu_{s}$ is also sensitive to the matrix strain 

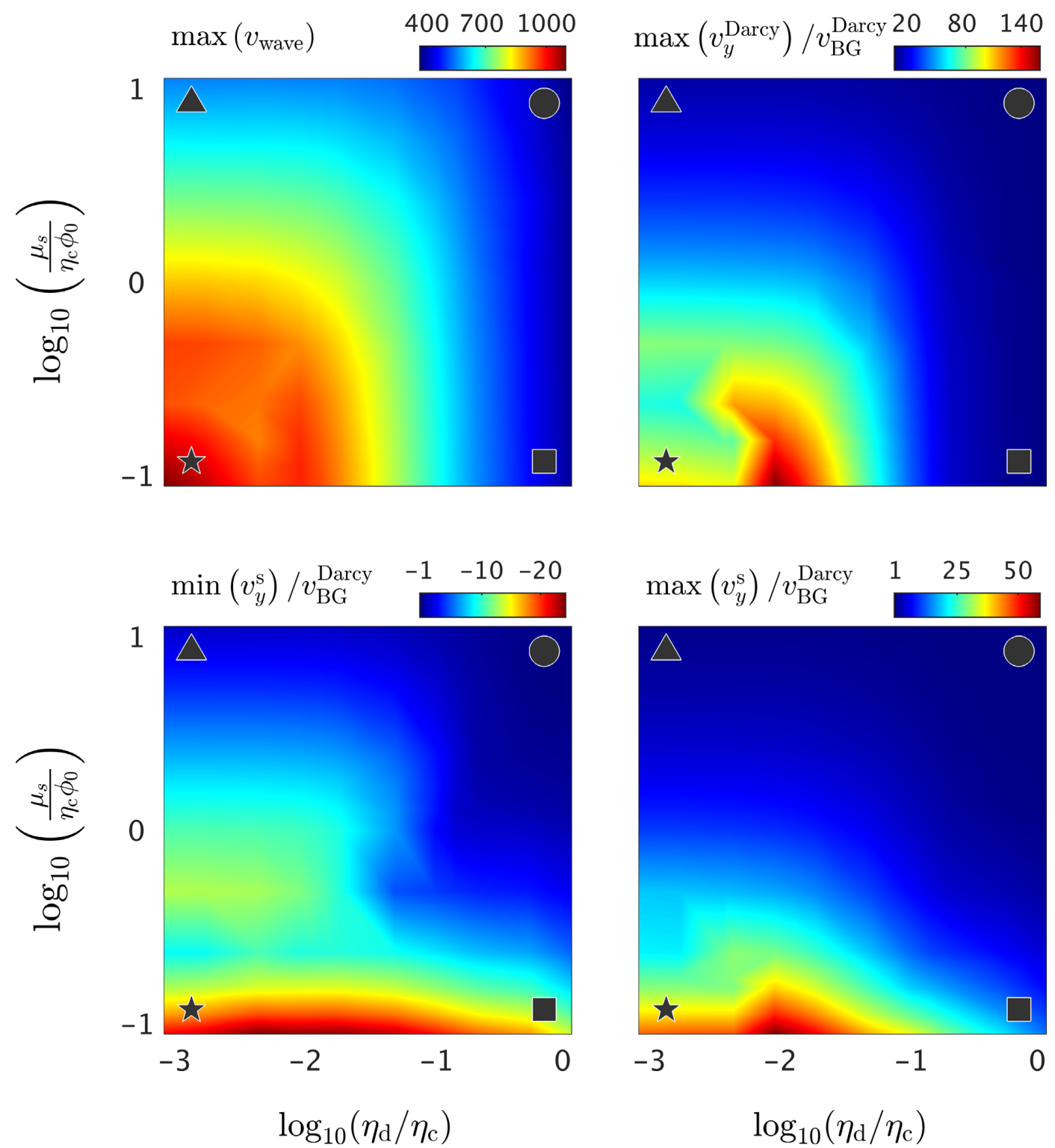

Figure 7. Systematic study of decompaction's relative importance over compaction bulk viscosity $1 / R=\eta_{\mathrm{d}} / \eta_{\mathrm{c}}$ (eq. 8 ) and shear over bulk viscosity $C=$ $\mu_{s} /\left(\eta_{\mathrm{c}} \phi_{0}\right)$ ratios (eq. 7). In the upper left-hand panel, we report maximal non-dimensional wave velocities max $\left(v_{\text {wave }}\right)$, scaled by the characteristic length $\left(\delta_{c}\right)$ and time $\left(\tau_{c}\right)$; in the upper right-hand panel, maximal Darcian fluxes max $\left(v_{y}^{\text {Darcy }}\right) / v_{\mathrm{BG}}^{\text {Darcy }}$; in the lower left-hand panel, the minimal solid velocities values $\min \left(v_{y}{ }^{\mathrm{s}}\right) / v_{\mathrm{BG}}^{\text {Darcy }}$; in the lower right panel, the maximal solid velocities values $\max \left(v_{y}^{\mathrm{s}}\right) / v_{\mathrm{BG}}^{\text {Darcy }}$. The symbols represent four end-member runs for which spatial distribution of $\log _{10}\left(\phi / \phi_{0}\right), \log _{10}\left(\max \left(v_{y}^{\text {Darcy }}\right) / v_{\mathrm{BG}}^{\text {Darcy }}\right)$ and $\log _{10}\left(\eta_{\phi} / \eta_{\mathrm{c}}\right)$ are reported in Figs 8,9 and 10 , respectively. The forward model used for this systematic study has a grid resolution of $511 \times 1023$ gridpoints in 2-D.

rate. The spatial distribution of $\left(\eta_{\phi} \phi_{0}\right) / \mu_{s}$ ratio (Fig. 12b) however confirms a more pronounced dependence of $\eta_{\phi}$ on $p_{e}$. This observation is in good agreement with the outcome of the systematical flow regimes investigated in 2-D.

\section{DISCUSSION}

\subsection{The genesis of high-porosity channels}

Numerically accurate resolving of coupled and non-linear twophase flow and including decompaction weakening as focussing mechanism allows one to predict the spontaneous formation and propagation of high-porosity channels (Fig. 2), developing from an initial region of elevated porosity, such as a fluid reservoir or a region containing partial melt. The buoyant fluid generates forces on the upper part of the reservoir, leading to increase in fluid pressure. The effective pressure decreases accordingly and decompaction occurs, permitting the fluid anomaly to move upwards. Pressure-sensitive bulk rheology further results in lower bulk viscosity values in the regions affected by decompaction, leading to enhanced upwardmigration. Decompaction weakening is responsible for orders of magnitude faster decompaction (opening of pores) at the top of the channels compared to compaction (closure of pores) at the tail of the channel. The fluid pressure gradient decreases from regions outside 

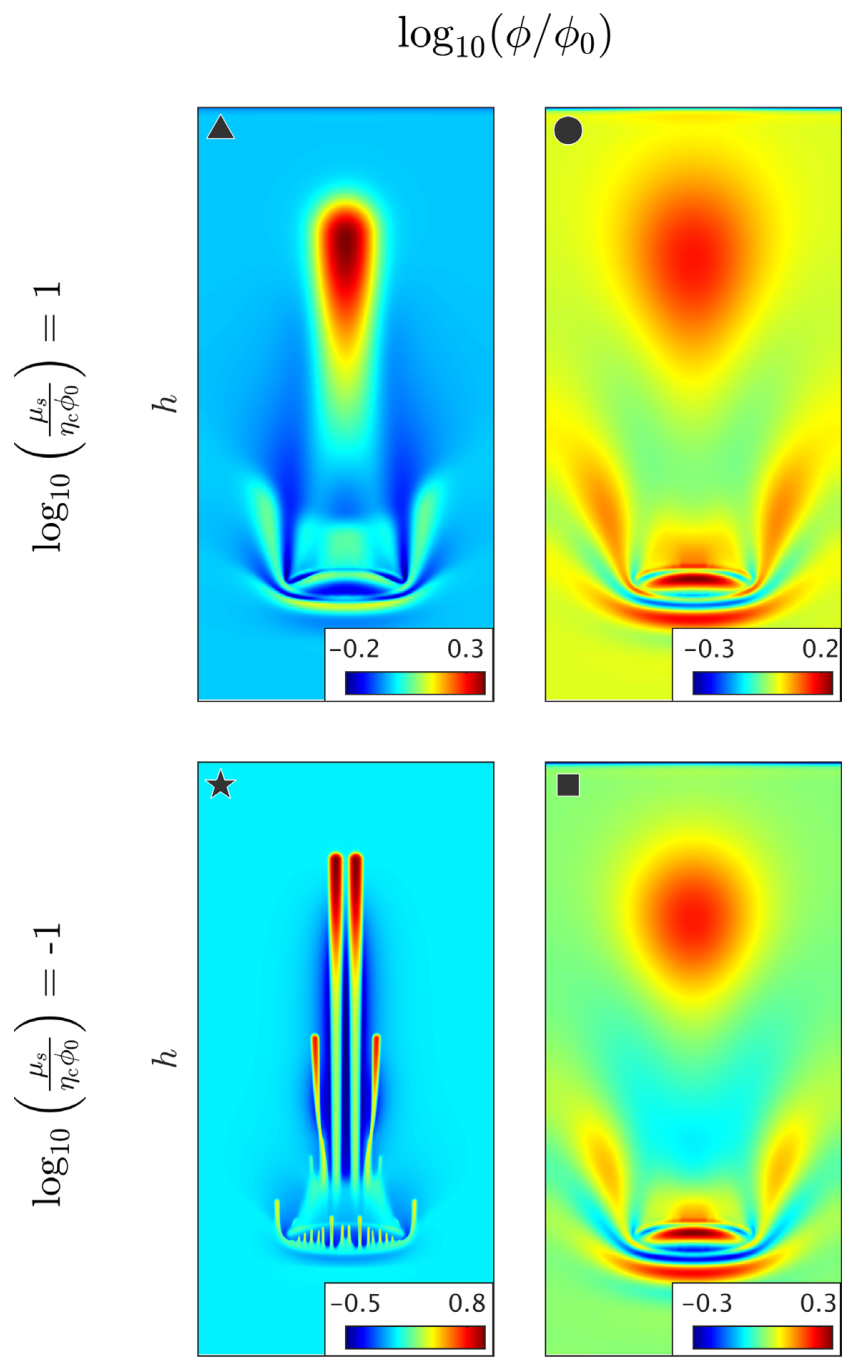

$x$

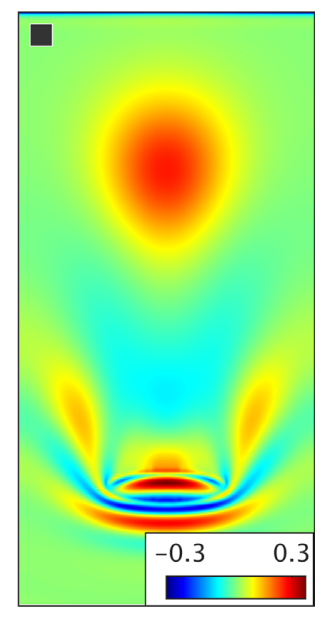

$x$

$$
\log _{10}\left(\eta_{\mathrm{d}} / \eta_{\mathrm{c}}\right)=-3 \quad \log _{10}\left(\eta_{\mathrm{d}} / \eta_{\mathrm{c}}\right)=0
$$

Figure 8. We report spatial distribution of normalized porosity $\log _{10}\left(\phi / \phi_{0}\right)$ for the end-member runs in the explored parameter space (Fig. 7). The final stage (i.e. when the porosity wave reached 85 per cent of the domain depth $h$ ) is shown with individual colour axes for every parameter combination to encounter the important variations between the different regimes.

the channels towards the inside of the channels, resulting in fluid drainage from the surrounding regions affected by the presence of a propagating channel. This mechanism enables the self-sustenance of the propagation of the porosity waves and results in fluid depletion in the rims of the channels. To ensure mass-balance, those rims thus compact and turn into high viscosity regions. Thus, the channels' structure may remain detectable even if the channels are dormant.

Both the channel spacing and the channel width are mainly controlled by the compaction length. Since we use a dimensionless implementation of the two-phase flow equations introducing characteristic scales (eq. 9), we can post-process the model outcome for various settings as long as the dependant variables conserve their respective ratios with regard to the defined scales. Thus, the three scales (eq. 9) can be used to predict the effective size and propagation speed of high-porosity channels for different reservoir

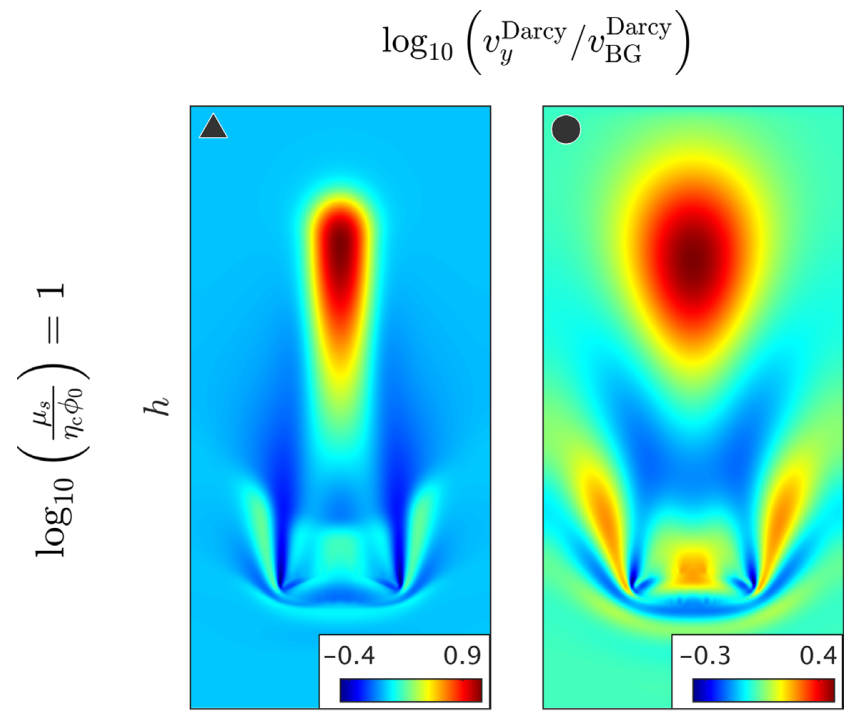

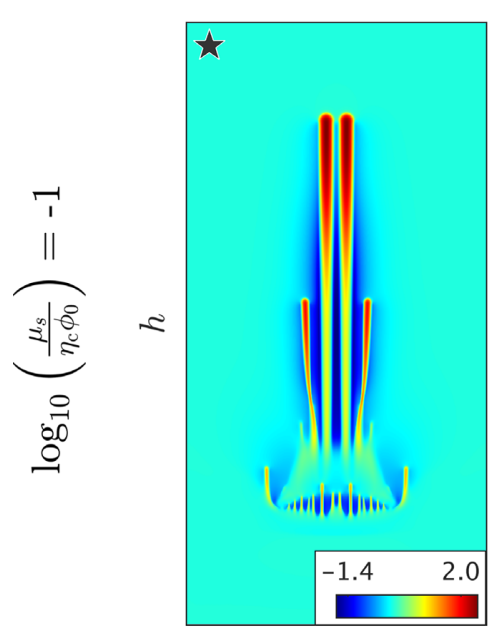

$x$

$$
\log _{10}\left(\eta_{\mathrm{d}} / \eta_{\mathrm{c}}\right)=-3 \quad \log _{10}\left(\eta_{\mathrm{d}} / \eta_{\mathrm{c}}\right)=0
$$

Figure 9. We report spatial distribution of normalized vertical Darcian flux $\log _{10}\left(v_{y}^{\text {Darcy }} / v_{\mathrm{BG}}^{\text {Darcy }}\right)$ for the end-member runs in the explored parameter space (Fig. 7). The final stage (i.e. when the porosity wave reached 85 per cent of the domain depth $h$ ) is shown with individual colour axes for every parameter combination to encounter the key variations between the different regimes.

and metamorphic environments, ranging from kilometre to metre size features, with propagation velocities in the range of metres per year in low-permeable shales to kilometres per years in permeable sandstones (Table 2).

\subsection{Prediction of localized flow regimes}

The power law relationship between maximal wave velocities and the combination of viscosity ratios (Fig. 11b) allows one to give an estimate on potential flow enhancement for relevant and welldefined rheological parameters. Further, a closer analysis of the various maximal velocity values shows a distinct trend, supporting the wave-like behaviour of porosity waves. The maximal dimensionless wave velocity is close to $10^{3}$. The maximal recorded vertical fluid 

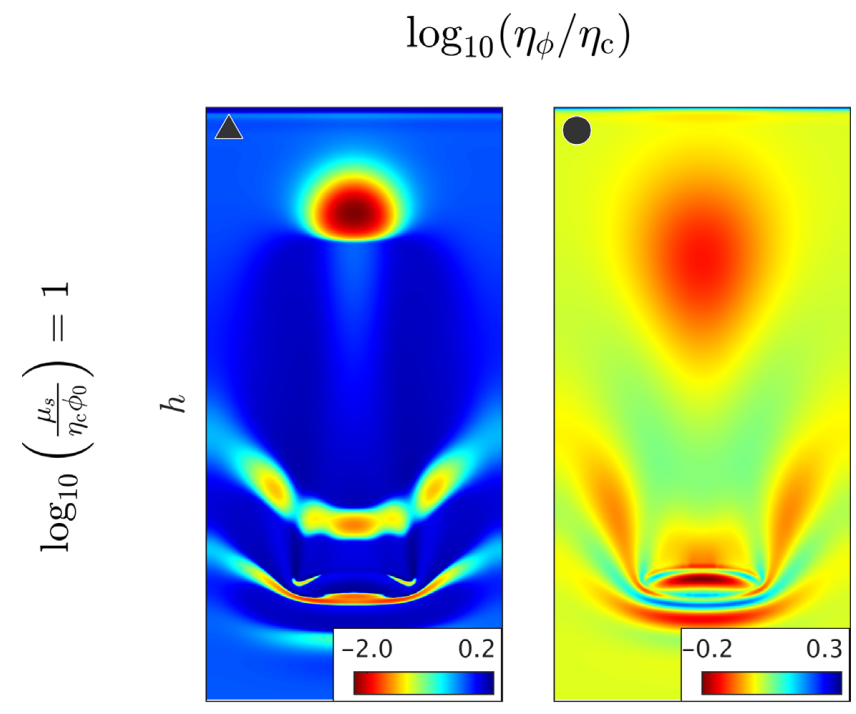

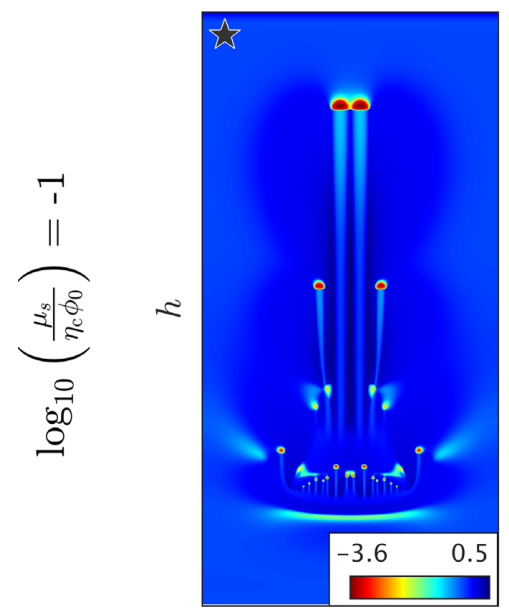

$x$

$$
\log _{10}\left(\eta_{\mathrm{d}} / \eta_{\mathrm{c}}\right)=-3 \quad \log _{10}\left(\eta_{\mathrm{d}} / \eta_{\mathrm{c}}\right)=0
$$

Figure 10. We report spatial distribution of normalized bulk viscosity $\log _{10}\left(\eta_{\phi} / \eta_{\mathrm{c}}\right)$ for the end-member runs in the explored parameter space (Fig. 7). The final stage (i.e. when the porosity wave reached 85 percent of the domain depth $h$ ) is shown with an individual colour axis for every parameter, to encounter the key variations between the different regimes. Notably, the colour axes are reversed for this figure; the red values represent low bulk viscosities — enhanced ability for fast viscous matrix deformation.

fluxes are about one order of magnitude lower, with values of close to 140 . The solid velocities are even one order of magnitude lower than the fluid fluxes; thus, two orders of magnitude lower than wave velocities. This clearly shows that fast-propagating high-porosity channels trigger minor solid displacement and moderate to high fluid fluxes. Our results highlight that the wave velocity is not the combination of the solid and fluid velocities, but the result from a more composite behaviour; the relatively small solid strain rates allow for local (de)compaction, while local fluid fluxes result from the active drainage of the passing wave. This mechanism continuously feeds the propagating wave which is thus growing over time. The fluid velocity within the wave's top part is slightly slower compared to the maximal wave motion speed. Reason for this discrepancy is that the wave is not only driven by the fluid contained in its interior but benefits from the active drainage. It is interesting to note the similarity in maximal positive and negative values for the solid velocity components' characteristics for percolation flow instead of circulation observed in diapirism regimes (Scott 1988).

Decompaction weakening is the principal driver owing to flow channeling in the reported results, suggested to be a viable mechanism leading to the formation of fluid escape pipes (Böttner et al. 2019). Porosity waves with a symmetric bulk rheology would fail to produce the vertically elongated tubular features strikingly well reproducing the subsurface flow features mapped and interpreted as fluid escape pipes (e.g. Løseth et al. 2011; Plaza-Faverola et al. 2011). We quantify by $R=\eta_{\mathrm{c}} / \eta_{\mathrm{d}}$ the level of asymmetry between compaction and decompaction creep regime; this parametrization reflects the occurrence of tensile micro-fractures developing in the regime where the fluid pore pressure $p^{f}$ overcomes the mean stress of the fluid-filled porous matrix $\bar{p}$. Comparing the decompaction weakening model versus results obtained using a viscoplastic matrix with dilatant brittle failure (Yarushina et al. 2015b, Section 3.4) suggests that a ratio of $\eta_{\mathrm{c}} / \eta_{\mathrm{d}} \approx 500$ corresponds in a first order to a tensile failure of the porous matrix at $Y / p_{c}=0.1$, where $Y$ stands for the failure property of the rock, here at about 10 per cent of the characteristic pressure $p_{c}$.

\subsection{Relevance of the hydromechanical model for geological applications}

The present study aims at an in-depth investigation of non-trivial outcomes owing to hydromechanical coupling. We, for the first time, implement the consistent set of two-phase flow equations proposed by Yarushina \& Podladchikov (2015a) in 2-D and 3-D and on very high spatial and temporal resolution. Within this contribution we systematically investigate the ability of diffusive fluid flow coupled to incompressible viscous porous matrix deformation to generate high-porosity channels in a spontaneous fashion without the need of any external and additional mechanisms. We report an enhanced focussing, narrower channels and faster wave velocities the lower the ratio $\mu_{s} /\left(\eta_{\mathrm{c}} \phi_{0}\right)$ is. In fact, some other physical processes may significantly influence the dynamic of the system, such as strain rates, temperature or chemical composition. The multiphysics couplings among those additional processes may further impact the shear and bulk viscosity. For exemple, a rise in temperature may tend to lower viscosity activating enhanced creep of the porous matrix. Chemical reactions may either consume or generate fluids and thus modify the mineral assemblages ultimately affecting the petrophysical properties of rocks. This study clearly suggests enhanced localized and focussed fluid flow for decreasing values of bulk and shear viscosity (Fig. 11).

The current model does yet not consider temperature neither chemical effects and our results mainly target shallow environments such as weakly consolidated sedimentary stacks saturated with non-reactive pore fluids. Nonetheless, the considered shallow rock formations we use as natural laboratory may experience considerable far-field tectonic stresses. We therefore augmented the model to account for strain rate dependent non-linear shear viscosity. Our preliminary 3-D results suggest the power law shear viscosity $\mu_{s}$ (for $n=3$ ) to act as a positive feedback mechanism that tends to lower $\mu_{s}$ in region of localized high strain rates, such as channels' tip (Fig. 12a). Far-field tectonic regimes may potentially play a key role in the channels and related pockmarks occurence leading towards preferred alignment patterns (Plaza-Faverola \& Keiding 2019). We 

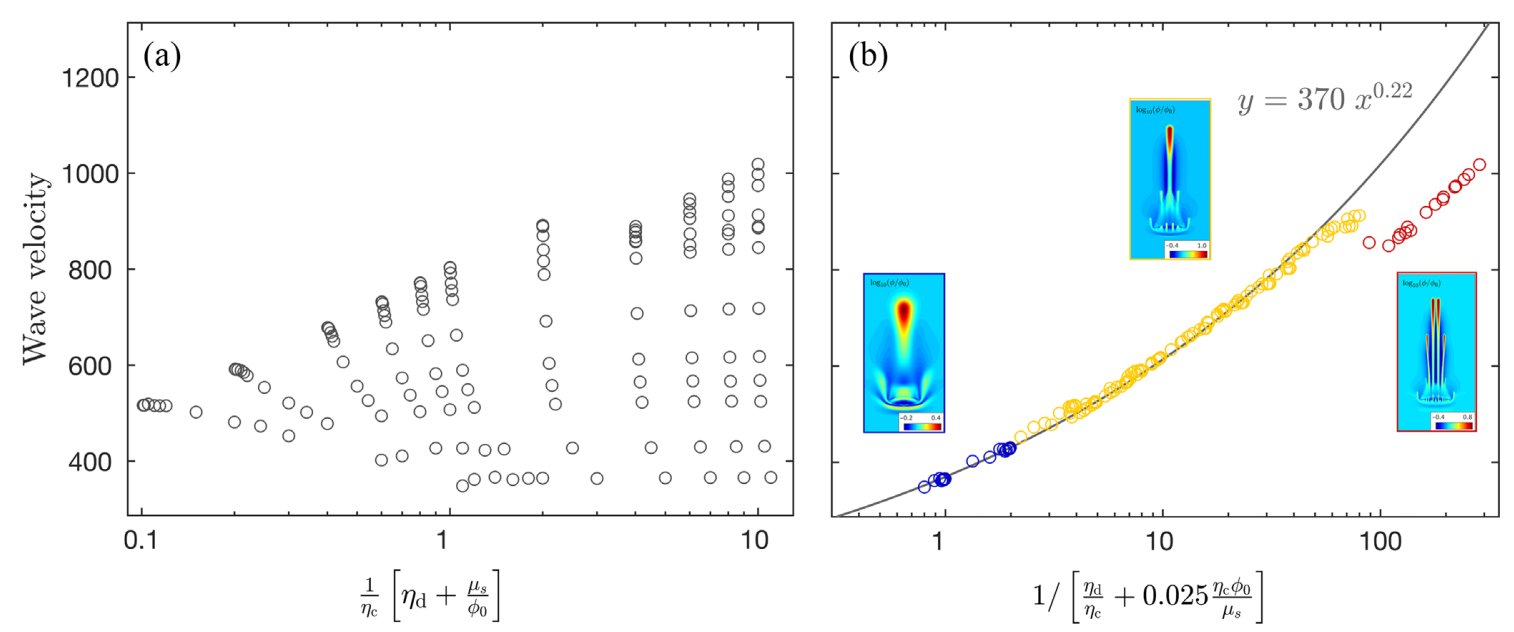

Figure 11. Wave velocity represented as function of combined systematically varied parameters, the bulk viscosity and bulk to shear viscosity ratios. (a) Wave velocity $\left(v_{\text {wave }}\right.$ ) plotted against linear combination of bulk decompaction $\eta_{\mathrm{d}}$ and solid shear viscosity $\mu_{s}$ normalized over compaction viscosity $\eta_{\mathrm{c}}$. (b) Wave velocity after data collapse: the formula shown as $x$-label allows us to collapse the entire systematic study result onto a single power law trend $y=370 x^{0.22}$. The three regimes are highlighted by colours, ranging from diffuse blobs to single-focussed channels, and finally, splitting into two channels towards the $x$-axis range end.

(a)

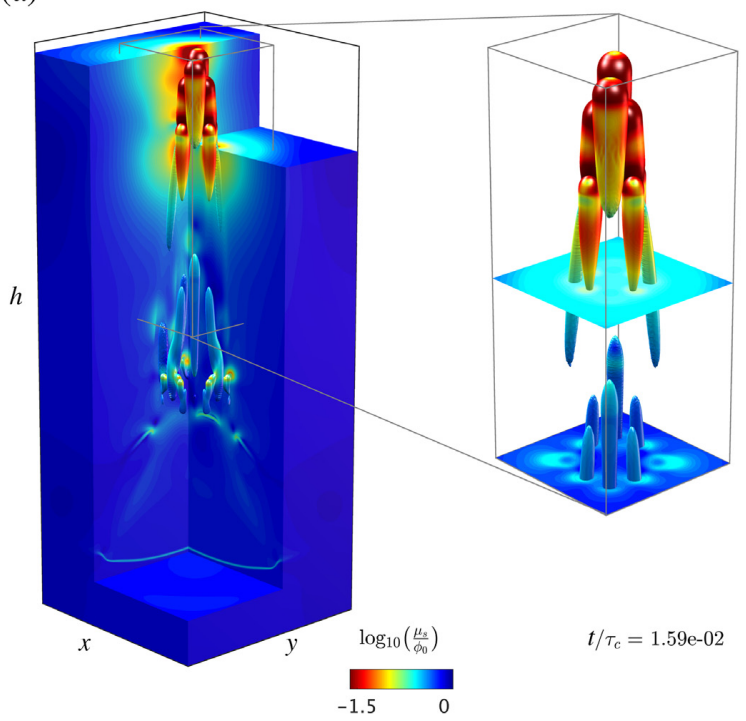

(b)

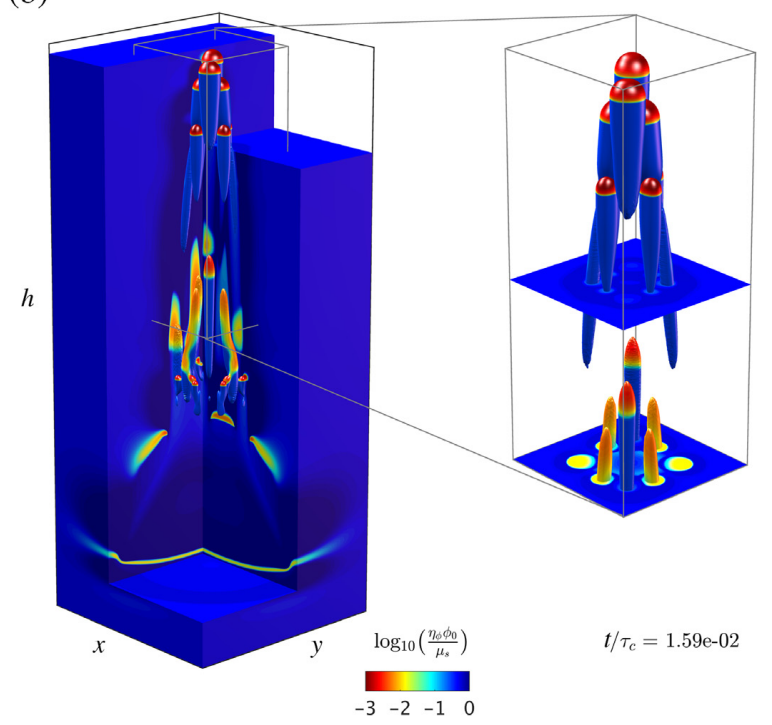

Figure 12. High-resolution numerical simulation of propagating high-porosity channels in 3-D. We report (a) the strain rate-dependant non-linear solid shear viscosity normalized over background porosity $\left(\mu_{s} / \phi_{0}\right)$ and (b) the non-linear bulk viscosity normalized over solid shear viscosity values $\left(\eta_{\phi} \phi_{0} / \mu_{s}\right)$. The initial condition used for this simulation is presented in Fig. 1(a) with $R=\eta_{\mathrm{c}} / \eta_{\mathrm{d}}=500$ and $C=\mu_{\mathrm{s}} /\left(\eta_{\mathrm{c}} \phi_{0}\right)=1$. The numerical resolution is $1023 \times 1023$ $\times 2048$ gridpoints in $3-\mathrm{D}$, which represents a total of $2 \times 10^{9}$ gridpoints. The subplots magnifie the central part of the computational domain $(290 \times 290$ $\times 760$ gridpoints) and provide a detailed view of (a) regions where elevated strain rates exhibit a close to two orders of magnitude decreases in solid shear viscosity values (of and around the developing fluid-conducting chimneys) and (b) the spatial distribution of bulk to shear viscosity ratios showing low values concentrated at the tip of the channels, highlighting the non-linear dependance of both bulk and shear viscosity on the effective pressure $\left(p_{e}\right)$. The simulation took three weeks to perform the close to $10^{4}$ time-steps on 128 Nvidia Titan X GPUs, using the entire octopus GPU-based supercomputer designed by the Scientific Computing Group and hosted by the Institute of Earth Sciences at the University of Lausanne. The tube-shaped isosurface contours 1.5 order of magnitude permeability increase from background values. The related movies are available as supplementary material.

preliminary investigate far-field extension, compression and strikeslip regimes within our 3-D numerical model in order to evaluate the impact of those major tectonic regimes on the high-porosity channel distribution (Figs 13a and b). We report that the channel distribution is sensitive to applied deformation regimes, while the channel geometry remains mostly unaffected (Figs 13a and b). 2-D vertical (Fig. 13c) and horizontal (Fig. 13d) cross-section for all three far-field regimes clearly illustrate that the vertical channel propagation (i.e. the vertical velocity) is maximal in extensional regimes, intermediate in shear regimes, and minimal in compressional regimes. Further, vertical channel connectivity seems maximal in horizontal shear regimes. Total pressure values reflect the mean stress state in the model. The results clearly show deviation from lithostatic pressure conditions as reported by the total pressure 

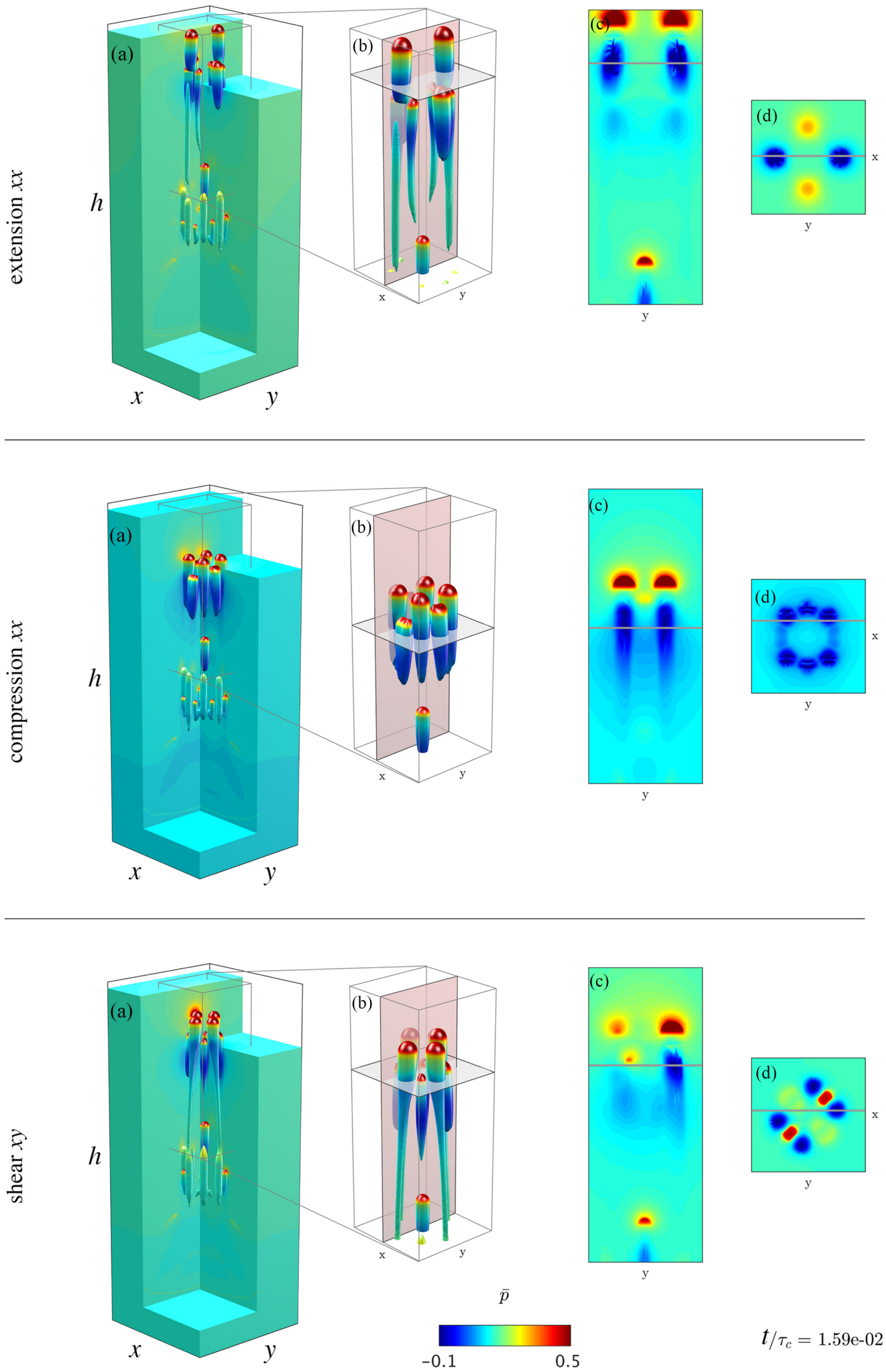

Figure 13. Total pressure $(\bar{p})$ distribution after dimensionless time $t=1.59 \times 10^{-2}$ for the 3-D configuration described in Fig. 12, as a function of three far-field tectonic regimes, namely extension along the $x$-axis, compression along the $x$-axis, and shear in the $x y$ horizontal plane. (a) Overview of the high-porosity channels' distribution. (b) Zoom-in on the region of interest, showing $\bar{p}$ values interpolated onto an isosurface of 1.5 order of magnitude increase in permeability. (c) $y z$ slice at $\mathrm{L}_{x} / 2$ of the (b) plot. (d) $x y$ slice at given depth $h$ as shown on (b). The related movie is available as Supporting Information. 
Table 2. Scaling of the numerical results to representative values for reservoir rocks (Räss et al. 2014, 2017b; Makhnenko \& Podladchikov 2018, and references therein) and crustal environments (McKenzie 1984).

\begin{tabular}{|c|c|c|c|c|c|}
\hline Desription & Shale & Limestone & Sandstone & Crustal rocks & Units \\
\hline Bulk viscosity $\left(\eta_{\phi}\right)$ & $10^{13}$ & $10^{15}$ & $10^{16}$ & $10^{16}$ & {$[\mathrm{~Pa} . \mathrm{s}]$} \\
\hline Permeability $\left(k_{\phi}\right)$ & $10^{-19}$ & $10^{-16}$ & $10^{-14}$ & $10^{-13}$ & {$\left[\mathrm{~m}^{2}\right]$} \\
\hline Fluid viscosity $\left(\mu_{f}\right)$ & $8 \times 10^{-4}$ & $8 \times 10^{-4}$ & $8 \times 10^{-4}$ & 1 & [Pa.s] \\
\hline Propagation speed & 0.3 & 300 & $10^{4}$ & 100 & {$\left[\mathrm{~m} \mathrm{yr}^{-1}\right]$} \\
\hline
\end{tabular}

$\bar{p}$ colormap. Changes in non-lithostatic pressure conditions in time and space may have major implications related to hydrochemical and reactions, such as methane hydrate expulsion within sedimentary basins.

We believe that these first results should be followed up with a detailed systematic analysis investigating the effects of shear deformation on the high-porosity channel formation and propagation before further building complexity into the model, such as adding metamorphic reactions, thermal effects and chemistry. We speculate however that these additional diffusive (non)linear multiphysics couplings may further enhance localization and a non-intuitive system behaviour (e.g. Omlin et al. 2017a), and require too be rigorously addressed in future studies.

\subsection{Benefits and limitations of the pseudo-transient method}

The pseudo-transient and direct-iterative solvers implemented both allow us to accurately resolve the non-linear coupled two-phase flow physics, in reasonable wall-time. Modern CPUs and the current amount of RAM present on modern desktop systems enable to compute numerical domains of up to 2 million gridpoints using DI solvers that are robust with the ability to handle large contrasts in material properties such as variable viscosity. Although the DI solver types are efficient in addressing 2-D models, such type of algorithms which necessitate the assembly of a large coefficient matrix may not be the optimal candidate when targeting very highresolution 3-D configurations.

The current development of hardware accelerators such as GPUs enables new possibilities for iterative methods. The massive number of arithmetic units of many-core computing devices such as GPUs allows one to perform computations at low costs. Further, the constant increase in memory bandwidth enables rapid memory transfers between on-chip memory and computing cores. Thus, these latest hardware developments put matrix-free iterative methods in the spotlight and make PT solvers fully competitive with matrix-based solvers, even on 2-D configurations (see Appendix D for additional details).

Since PT-based solvers rely on finite-difference stencils to evaluate partial derivatives, the memory access patterns remain fairly local and the memory usage grows linearly with increasing problem size. The addition of point-to-point MPI communication directly enables the PT solvers to run on distributed-memory supercomputers, mandatory when targeting high-resolution 3-D calculations. Moreover, appropriate communication and computation overlap permits to achieve close-to-optimal parallel efficiency. Thus, we were able to produce the 3-D results (Section 5.4) in less than 3 weeks, calculating more than $10^{4}$ time-steps on more than $2 \times 10^{9}$ gridpoints. We involved 128 Titan X GPUs available in the octopus GPU-based supercomputer, hosted by the Swiss Geocomputing Centre at the Institute of Earth Sciences, University of Lausanne (Appendix D).

In summary, the key benefits of the PT method are as follows: (i) we obtain identical results as the DI method, (ii) the implementation of PT solver types using the finite-difference method is straightforward and results in short, readable codes (see the MATLAB PT codes in the supplementary material), (iii) the GPU implementation of PT solvers is fully competitive compared to the DI approach, and iv) PT solvers are a viable option when investigating 3-D configurations; further, MPI parallelization can readily be implemented for distributed-memory parallelization. Finally, the approach's limitations reside mainly in deprecated convergence rates for large contrasts in material properties over narrow spatial extent and the need for additional effort finding optimal numerical parameters for highly non-linear configurations.

\section{CONCLUSIONS}

We successfully developed 2-D and 3-D routines to solve coupled hydromechanical problems. We benchmarked both the pseudotransient-based and direct-iterative-based two-phase flow-solvers and report their relative sensitivity on the non-linear threshold and the numerical grid resolution. We conclude that both methods are in agreement, since the GPU-based pseudo-transient matrix-free solver and the CPU-based direct-iterative solver unveil identical results. We herewith confirm similar trends as observed in a companion study investigating thermo-mechanical coupling in 2-D and 3-D (Duretz et al. 2019).

We further highlight the strong impacts of non-sufficient grid resolution and non-linear threshold on physical results after a large number of time-steps. We performed a systematic investigation of hydromechanical coupled flow in deforming porous media. The outcome of the data collapse is a scaling power law that predicts the propagating wave velocity as a combination of viscous bulk and shear rheology.

We also show that the MPI-based and GPU-based implementation of the PT routines allowed us to realize extremely high-resolution 3 -D simulations involving over $2 \times 10^{9}$ gridpoints with a parallel efficiency close to 1 . This significant increase in affordable resolution enabled us to tackle mega-pixel resolution in all three dimensions. The 3-D results provide novel insights towards better understanding the physics of chimney formation. Predicting with high accuracy the potential localization of flow in the shallow subsurface may support reliable risk assessment related to underground waste storage operations (e.g. Räss et al. 2018).

\section{ACKNOWLEDGEMENTS}

The authors thank Nina Simon, Stefan Schmalholz and Viktoryia Yarushina for enlightening discussion during the entire project, and 
are grateful to Samuel Omlin for his participation in the early stage design of the HPC application related to the current research. We acknowledge Philippe Logean for his constant support and development with the GPU-based octopus supercomputer. We thank two anonymous reviewers which provided valuable feedback that permitted to significantly enhance the focus of the manuscript.

\section{REFERENCES}

Ague, J.J., 2011. Extreme channelization of fluid and the problem of element mobility during Barrovian metamorphism, Am. Mineral., 96(2-3), 333352.

Aharonov, E., Whitehead, J., Kelemen, P. \& Spiegelman, M., 1996. Channeling instability of upwelling melt in the mantle, Oceanogr. Lit. Rev., 8(43), 798.

Appold, M.S. \& Nunn, J.A., 2002. Numerical models of petroleum migration via buoyancy-driven porosity waves in viscously deformable sediments, Geofluids, 2(3), 233-247.

Barcilon, V. \& Richter, F.M., 1986. Non-linear waves in compacting media, J. Fluid Mech., 164(1), 429.

Baud, P., Schubnel, A. \& Wong, T.-F.. 2000. Dilatancy, compaction, and failure mode in solnhofen limestone, J. geophys. Res.: Solid Earth, 105(B8), 19289-19303.

Berndt, C., 2005. Focused fluid flow in passive continental margins, Phil. Trans. R. Soc. Lond. A: Math., Phys. Eng. Sci., 363(1837), 2855-2871.

Böttner, C. et al., 2019. Pockmarks in the Witch Ground Basin, Central North Sea, Geochem. Geophys. Geosyst., 20(4), 1698-1719.

Brantut, N., Heap, M., Meredith, P. \& Baud, P., 2013. Time-dependent cracking and brittle creep in crustal rocks: a review, J. Struct. Geol., 52, $17-43$.

Cacace, M. \& Jacquey, A.B., 2017. Flexible parallel implicit modelling of coupled thermal-hydraulic-mechanical processes in fractured rocks, Solid Earth, 8(5), 921.

Cai, Z. \& Bercovici, D., 2013. Two-phase damage models of magmafracturing, Earth planet. Sci. Lett., 368, 1-8.

Cartwright, J. \& Santamarina, C., 2015. Seismic characteristics of fluid escape pipes in sedimentary basins: implications for pipe genesis, Mar. Petrol. Geol., 65, 126-140.

Cathles, L., Su, Z. \& Chen, D., 2010. The physics of gas chimney and pockmark formation, with implications for assessment of seafloor hazards and gas sequestration, Mar. Petrol. Geol., 27(1), 82-91.

Connolly, J. \& Podladchikov, Y., 2007. Decompaction weakening and channeling instability in ductile porous media: Implications for asthenospheric melt segregation, J. geophys. Res.: Solid Earth, 112(B10), doi:10.1029/2005JB004213.

Connolly, J. \& Podladchikov, Y.Y., 1998. Compaction-driven fluid flow in viscoelastic rock, Geodin. Acta, 11(2-3), 55-84.

Connolly, J. \& Podladchikov, Y.Y., 2000. Temperature-dependent viscoelastic compaction and compartmentalization in sedimentary basins, Tectonophysics, 324(3), 137-168.

Connolly, J.A.D. \& Podladchikov, Y.Y., 2014 An analytical solution for solitary porosity waves: dynamic permeability and fluidization of nonlinear viscous and viscoplastic rock, Geofluids, 15(1-2), 269-292.

Costa, A., 2006. Permeability-porosity relationship: a reexamination of the Kozeny-Carman equation based on a fractal pore-space geometry assumption, Geophys. Res. Lett., 33(2), L02318.

Cundall, P., Coetzee, M., Hart, R. \& Varona, P., 1993. Flac user's manual, Itasca Consulting Group, USA.

Cundall, P.A., 1982. Adaptive density-scaling for time-explicit calculations, in Proceedings of the 4th Int. Con. on Numerical Methods in Geomechanics, Edmonton, S, pp. 23-26.

Cundall, P.A., 1987. Distinct element models of rock and soil structure, in Analytical and Computational Methods in Engineering Rock Mechanics, Allen \& Unwin , pp. 129-163.

Cundall, P.A. \& Strack, O.D., 1979. A discrete numerical model for granular assemblies, geotechnique, 29(1), 47-65.
Dannberg, J. \& Heister, T., 2016. Compressible magma/mantle dynamics: 3D, adaptive simulations in ASPECT, J. geophys. Int., 207(3), 1343-1366.

David, C., Dautriat, J., Sarout, J., Delle Piane, C., Menéndez, B., Macault, R. \& Bertauld, D., 2015. Mechanical instability induced by water weakening in laboratory fluid injection tests, J. geophys. Res.: Solid Earth, 120(6), 4171-4188.

Duretz, T., Räss, L., Podladchikov, Y. \& Schmalholz, S., 2019. Resolving thermomechanical coupling in two and three dimensions: spontaneous strain localization owing to shear heating, J. geophys. Int., 216(1), 365379.

Dymkova, D. \& Gerya, T., 2013. Porous fluid flow enables oceanic subduction initiation on earth, Geophys. Res. Lett., 40(21), 5671-5676.

Elenius, M. et al., 2018. Assessment of $\mathrm{CO}_{2}$ storage capacity based on sparse data: Skade Formation, Int. J. Greenhouse Gas Contl., 79, 252-271.

Frankel, S.P., 1950. Convergence rates of iterative treatments of partial differential equations, Math. Tables Other Aids Comput., 4(30), 65-75.

Gradmann, S. \& Beaumont, C., 2012. Coupled fluid flow and sediment deformation in margin-scale salt-tectonic systems: 2. Layered sediment models and application to the northwestern Gulf of Mexico, Tectonics, 31(4), doi:10.1029/2011TC003035.

Gradmann, S., Beaumont, C. \& Ings, S.J., 2012. Coupled fluid flow and sediment deformation in margin-scale salt-tectonic systems: 1. Development and application of simple, single-lithology models, Tectonics, 31(4), doi:10.1029/2011TC003033.

Haga, J.B., Osnes, H. \& Langtangen, H.P., 2012. A parallel block preconditioner for large-scale poroelasticity with highly heterogeneous material parameters, Comput. Geosci., 16(3), 723-734.

Hawkins, C., Angheluta, L., Krotkiewski, M. \& Jamtveit, B., 2016. Reynolds-number dependence of the longitudinal dispersion in turbulent pipe flow, Phys. Rev. E, 93, 043119.

Huq, F., Smalley, P.C., Mørkved, P.T., Johansen, H., Johansen, I. \& Yarushina, V., 2017. The Longyearbyen $\mathrm{CO}_{2}$ lab : fluid communication in reservoir and caprock, Int. J. Greenhouse Gas Contl., 63, 59-76.

Hustoft, S., Bünz, S. \& Mienert, J., 2010. Three-dimensional seismic analysis of the morphology and spatial distribution of chimneys beneath the nyegga pockmark field, offshore mid-norway, Basin Res., 22(4), 465-480.

Iyer, K., Rüpke, L. \& Galerne, C.Y., 2013. Modeling fluid flow in sedimentary basins with sill intrusions: implications for hydrothermal venting and climate change, Geochem. Geophys. Geosyst., 14(12), 5244-5262.

Joshi, A. \& Appold, M.S., 2016. Potential of porosity waves for methane transport in the Eugene Island field of the Gulf of Mexico basin, Mar. Petrol. Geol., 75, 1-13.

Judd, A. \& Hovland, M., 2007. Seabed Fluid Flow, Cambridge University Press.

Katz, R.F. \& Weatherley, S.M., 2012. Consequences of mantle heterogeneity for melt extraction at mid-ocean ridges, Earth planet. Sci. Lett., 335-336, 226-237.

Keller, T., May, D.A. \& Kaus, B. J.P., 2013. Numerical modelling of magma dynamics coupled to tectonic deformation of lithosphere and crust, $J$. geophys. Int., 195(3), 1406-1442.

Keller, T., Katz, R.F. \& Hirschmann, M.M., 2017. Volatiles beneath midocean ridges: deep melting, channelized transport, focusing, and metasomatism, Earth planet. Sci. Lett., 464, 55-68.

Kelley, C.T. \& Keyes, D.E., 1998. Convergence analysis of pseudo-transient continuation, SIAM J. Numer. Anal., 35(2), 508-523.

Kelley, C.T. \& Liao, L.-Z., 2013. Explicit pseudo-transient continuation, Pacific J.Opt., 9(1), 77-91.

Lewis, R. \& Schrefler, B., 1987. The Finite Element Method in the Deformation and Consolidation of Porous Media, John Wiley and Sons Inc.

Liang, Y., Schiemenz, A., Hesse, M.A. \& Parmentier, E.M., 2011. Waves, channels, and the preservation of chemical heterogeneities during melt migration in the mantle, Geophys. Res. Lett., 38(20), 1-5.

Liu, W., Yao, J., Chen, Z. \& Liu, Y., 2015. Effect of quadratic pressure gradient term on a one-dimensional moving boundary problem based on modified Darcy's law, Acta Mech. Sin., 32 (1), 38-53.

Løseth, H., Wensaas, L., Arntsen, B., Hanken, N.-M., Basire, C. \& Graue, K., 2011. $1000 \mathrm{~m}$ long gas blow-out pipes, Mar. Petrol. Geol., 28(5), $1047-1060$. 
Makhnenko, R.Y. \& Labuz, J.F., 2016. Elastic and inelastic deformation of fluid-saturated rock, Phil. Trans. R. Soc. A, 374(2078), .

Makhnenko, R.Y. \& Podladchikov, Y.Y., 2018. Experimental poroviscoelasticity of common sedimentary rocks, J. geophys. Res.: Solid Earth, 123(9), 7586-7603.

Malvoisin, B., Podladchikov, Y.Y. \& Vrijmoed, J.C., 2015. Coupling changes in densities and porosity to fluid pressure variations in reactive porous fluid flow: local thermodynamic equilibrium, Geochem. Geophys. Geosyst., 16(12), 4362-4387.

Mazzini, A. et al., 2017. A climatic trigger for the giant Troll pockmark field in the northern North Sea, Earth planet. Sci. Lett., 464, 24-34.

McKee, S., Tomé, M., Ferreira, V., Cuminato, J., Castelo, A., Sousa, F. \& Mangiavacchi, N., 2008. The MAC method, Comput. Fluids, 37(8), 907-930.

McKenzie, D., 1984. The generation and compaction of partially molten rock, J. Petrol., 25(3), 713-765.

Miller, S.A., van der Zee, W., Olgaard, D.L. \& Connolly, J. A.D., 2003. A fluid-pressure feedback model of dehydration reactions: experiments, modelling, and application to subduction zones, Tectonophysics, 370(1), 241-251.

Minakov, A., Yarushina, V., Faleide, J.I., Krupnova, N., Sakoulina, T., Dergunov, N. \& Glebovsky, V., 2017. Dyke emplacement and crustal structure within a continental large igneous province, northern Barents Sea, Geol. Soc., Lond., Spec. Publ., SP460.4.

Minkoff, S.E., Stone, C., Bryant, S., Peszynska, M. \& Wheeler, M.F., 2003. Coupled fluid flow and geomechanical deformation modeling, J. Petrol. Sci. Eng., 38(1-2), 37-56.

Morency, C., Huismans, R.S., Beaumont, C. \& Fullsack, P., 2007. A numerical model for coupled fluid flow and matrix deformation with applications to disequilibrium compaction and delta stability, J. geophys. Res.: Solid Earth, 112(B10), doi:10.1029/2006JB004701.

Oliveira, B., Afonso, J.C., Zlotnik, S. \& Diez, P., 2017. Numerical modelling of multiphase multicomponent reactive transport in the Earth's interior, J. geophys. Int., 212(1), 345-388.

Olson, P. \& Christensen, U., 1986. Solitary wave propagation in a fluid conduit within a viscous matrix, J. geophys. Res.: Solid Earth, 91(B6), 6367-6374.

Omlin, S., 2017. Development of massively parallel near peak performance solvers for three-dimensional geodynamic modelling., Ph.D. thesis, University of Lausanne.

Omlin, S., Malvoisin, B. \& Podladchikov, Y.Y., 2017a. Pore fluid extraction by reactive solitary waves in 3-D, Geophys. Res. Lett., 44 (18), 9267-9275.

Omlin, S., Räss, L. \& Podladchikov, Y.Y., 2017b. Simulation of threedimensional viscoelastic deformation coupled to porous fluid flow, Tectonophysics, 746, 695-701.

Otter, J.R.H., Cassell, A.C. \& Hobbs, R.E., 1966. Dynamic relaxation, Proc. Inst. Civil Eng., 35(4), 633-656.

Patankar, S., 1980. Numerical Heat Transfer and Fluid Flow, CRC Press.

Phillips, P.J. \& Wheeler, M.F., 2008. A coupling of mixed and discontinuous galerkin finite-element methods for poroelasticity, Comput. Geosci., 12(4), 417-435.

Plaza-Faverola, A. \& Keiding, M., 2019. Correlation between tectonic stress regimes and methane seepage on the western Svalbard margin, Solid Earth, 10(1), 79-94.

Plaza-Faverola, A., Bünz, S. \& Mienert, J., 2010. Fluid distributions inferred from p-wave velocity and reflection seismic amplitude anomalies beneath the nyegga pockmark field of the mid-norwegian margin, Mar. Petrol. Geol., 27(1), 46-60.

Plaza-Faverola, A., Bünz, S. \& Mienert, J., 2011. Repeated fluid expulsion through sub-seabed chimneys offshore norway in response to glacial cycles, Earth planet. Sci. Lett., 305(3), 297-308.

Plümper, O., John, T., Podladchikov, Y.Y., Vrijmoed, J.C. \& Scambelluri, M., 2017. Fluid escape from subduction zones controlled by channel-forming reactive porosity, Nat. Geosci., 10(2), 150-156.

Poliakov, A.N.B., Cundall, P.A., Podladchikov, Y.Y. \& Lyakhovsky, V.A., 1993. An Explicit Inertial Method for the Simulation of Viscoelastic Flow: An Evaluation of Elastic Effects on Diapiric Flow in Two- and ThreeLayers Models, pp. 175-195, Springer.
Prevost, J.H., 2013. One-way versus two-way coupling in reservoirgeomechanical models, in Poromechanics V: Proceedings of the Fifth Biot Conference on Poromechanics, pp. 517-526, American Society of Civil Engineers, doi:10.1061/9780784412992.061.

Räss, L., Yarushina, V.M., Simon, N.S. \& Podladchikov, Y.Y., 2014. Chimneys, channels, pathway flow or water conducting features-an explanation from numerical modelling and implications for $\mathrm{CO}_{2}$ storage, Ener. Proc., 63, 3761-3774.

Räss, L., Yarushina, V., Duretz, T. \& Podladchikov, Y., 2016. High-resolution numerical modelling to resolve the dynamics of pipe structures in porous media, in ECMOR XV-15th European Conference on the Mathematics of Oil Recovery, doi:10.3997/2214-4609.201601746, EAGE, http://www. earthdoc.org/publication/publicationdetails/?publication=86194.

Räss, L., Duretz, T., Podladchikov, Y.Y. \& Schmalholz, S.M., 2017a. M2Di: concise and efficient MATLAB 2-D stokes solvers using the finite difference method, Geochem. Geophys. Geosyst., 18(2), 755-768.

Räss, L., Makhnenko, R.Y., Podladchikov, Y. \& Laloui, L., 2017b. Quantification of viscous creep influence on storage capacity of caprock, Ener. Proc., 114, 3237-3246.

Räss, L., Simon, N.S. \& Podladchikov, Y.Y., 2018. Spontaneous formation of fluid escape pipes from subsurface reservoirs, Scient. Rep., 8(1), 11116.

Reusch, A. et al., 2015. Giant lacustrine pockmarks with subaqueous groundwater discharge and subsurface sediment mobilization, Geophys. Res. Lett., 42(9), 3465-3473.

Rhebergen, S., Wells, G.N., Wathen, A.J. \& Katz, R.F., 2015. Three-field block preconditioners for models of coupled magma/mantle dynamics, SIAM J. Scient. Comput., 37(5), A2270-A2294.

Rozhko, A.Y., 2008. Benchmark for poroelastic and thermoelastic numerical codes, Phys. Earth planet. Inter., 171(1), 170-176.

Rudge, J., 2014. Analytical solutions of compacting flow past a sphere, $J$. Fluid Mech., 746, 466-497.

Rudge, J.F., Bercovici, D. \& Spiegelman, M., 2011. Disequilibrium melting of a two phase multicomponent mantle, J. geophys. Int., 184(2), 699-718.

Rutqvist, J., 2011. Status of the tough-flac simulator and recent applications related to coupled fluid flow and crustal deformations, Comput. Geosci., 37(6), 739-750.

Rutqvist, J., 2012. The geomechanics of co2 storage in deep sedimentary formations, Geotech. Geol. Eng., 30(3), 525-551.

Schiemenz, A., Liang, Y. \& Parmentier, E.M., 2011. A high-order numerical study of reactive dissolution in an upwelling heterogeneous mantle-I. Channelization, channel lithology and channel geometry, J. geophys. Int., 186(2), 641-664.

Scott, D.R., 1988. The competition between percolation and circulation in a deformable porous medium, J. geophys. Res.: Solid Earth, 93(B6), 64516462.

Scott, D.R. \& Stevenson, D.J., 1984. Magma solitons, Geophys. Res. Lett., 11(11), 1161-1164.

Settari, A., Walters, D.A. et al., 2001. Advances in coupled geomechanical and reservoir modeling with applications to reservoir compaction, SPE J., 6(03), 334-342.

Shin, D. \& Strikwerda, J.C., 1997. Inf-sup conditions for finite-difference approximations of the stokes equations, J. Aust. Math. Soc., B. Appl. Math., 39(01), 121-134.

Simpson, G., Spiegelman, M. \& Weinstein, M.I., 2010a. A multiscale model of partial melts: 1. Effective equations, J. geophys. Res., 115(B4), B04410.

Simpson, G., Spiegelman, M. \& Weinstein, M.I., 2010b. A multiscale model of partial melts: 2. Numerical results, J. geophys. Res., 115(B4), B04411.

Skarbek, R.M. \& Rempel, A.W., 2016. Dehydration-induced porosity waves and episodic tremor and slip, Geochem. Geophys. Geosyst., 17(2), 442469.

Skjetne, E. \& Auriault, J., 1999. New insights on steady, non-linear flow in porous media, Eur. J. Mech. - B/Fluids, 18(1), 131-145.

Sone, H. \& Zoback, M.D., 2014. Time-dependent deformation of shale gas reservoir rocks and its long-term effect on the in situ state of stress, Int. J. Rock Mech. Mining Sci., 69, 120-132.

Spiegelman, M. \& Kelemen, P.B., 2003. Extreme chemical variability as a consequence of channelized melt transport, Geochem. Geophys. Geosyst., 4(7), doi:10.1029/2002GC000336. 
Spiers, C., Schutjens, P., Brzesowsky, R., Peach, C., Liezenberg, J. \& Zwart, H., 1990. Experimental determination of constitutive parameters governing creep of rocksalt by pressure solution, Geological Society, Lond., Spec. Publ., 54(1), 215-227.

Stevenson, D.J. \& Scott, D.R., 1991. Mechanics of fluid-rock systems, Ann. Rev. Fluid Mech., 23(1), 305-339.

Tian, M. \& Ague, J.J., 2014. The impact of porosity waves on crustal reaction progress and $\mathrm{CO}_{2}$ mass transfer, Earth planet. Sci. Lett., 390, 80-92.

Vajdova, V., Baud, P., Wu, L. \& Wong, T.-F., 2012. Micromechanics of inelastic compaction in two allochemical limestones, J. Struct. Geol., 43, $100-117$.

Virieux, J., 1986. P-SV wave propagation in heterogeneous media: velocitystress finite-difference method, Geophysics, 51(4), 889-901.

Wang, H., 2000. Theory of Linear Poroelasticity with Applications to Geomechanics and Hydrogeology, Princeton University Press.

Wheeler, M., Xue, G. \& Yotov, I., 2014. Coupling multipoint flux mixed finite element methodswith continuous galerkin methods for poroelasticity, Comput. Geosci., 18(1), 57-75.

Wiggins, C. \& Spiegelman, M., 1995. Magma migration and magmatic solitary waves in 3-D, Geophys. Res. Lett., 22(10), 1289-1292.

Wong, T.-F. \& Baud, P., 2012. The brittle-ductile transition in porous rock: a review, J. Struct. Geol., 44, 25-53.

Yang, X.I. \& Mittal, R., 2014. Acceleration of the Jacobi iterative method by factors exceeding 100 using scheduled relaxation, J. Comput. Phys., 274, 695-708.

Yarushina, V.M. \& Podladchikov, Y.Y., 2015a. (de) compaction of porous viscoelastoplastic media: model formulation, J. geophys. Res.: Solid Earth, 120(6), 4146-4170.

Yarushina, V.M., Podladchikov, Y.Y. \& Connolly, J.A., 2015b. (de) compaction of porous viscoelastoplastic media: solitary porosity waves, $J$. geophys. Res.: Solid Earth, 120(7), 4843-4862.

Zheng, L., May, D., Gerya, T. \& Bostock, M., 2016. Fluid-assisted deformation of the subduction interface: coupled and decoupled regimes from 2-D hydromechanical modeling, J. geophys. Res.: Solid Earth, 121(8), 6132-6149.

\section{SUPPORTING INFORMATION}

Supplementary data are available at $G J I$ online.

\section{HM_GJI_Codes.zip,HM_GJI_SI.zip}

Please note: Oxford University Press is not responsible for the content or functionality of any supporting materials supplied by the authors. Any queries (other than missing material) should be directed to the corresponding author for the paper.

\section{APPENDIX A: THE DIRECT-ITERATIVE METHOD}

\section{A1 The direct-iterative scheme}

The implicit (or semi-implicit) discretization of the hydromechanical equations result in a linear system of equations such as:

$\mathbf{K}_{\mathrm{HM}} \mathbf{x}=\mathbf{b}$,

and couples the different solutions variables $\mathbf{v}, \overline{\mathbf{p}}, \mathbf{p}^{\mathbf{f}}$ as following:

$$
\underbrace{\left[\begin{array}{ccc}
\mathbf{K}_{\mathrm{vv}} & \mathbf{K}_{\mathrm{vp}} & \mathbf{0} \\
\mathbf{K}_{\mathrm{pv}} & \mathbf{K}_{\mathrm{pp}} & \mathbf{K}_{\mathrm{ppf}} \\
\mathbf{0} & \mathbf{K}_{\mathrm{pfp}} & \mathbf{K}_{\mathrm{pf}}
\end{array}\right]}_{\mathbf{K}_{\mathrm{HM}}} \underbrace{\left[\begin{array}{c}
\mathbf{v} \\
\overline{\mathbf{p}} \\
\mathbf{p}^{\mathbf{f}}
\end{array}\right]}_{\mathbf{x}}=\underbrace{\left[\begin{array}{c}
\mathbf{b}_{\mathrm{v}} \\
\mathbf{b}_{\overline{\mathrm{p}}} \\
\mathbf{b}_{\mathrm{p}^{\mathrm{f}}}
\end{array}\right]}_{\mathbf{b}}
$$

where the matrix $\mathbf{K}_{\mathrm{HM}}$ contains discrete coefficients (staggered finite differences). The symmetric and positive-defined $\mathbf{K}_{\mathrm{Vv}}$ block represents the mechanical operator. The block $\mathbf{K}_{\mathrm{pv}}$ represents the divergence operator, which equals minus the transposed of the gradient operator $\mathbf{K}_{\mathrm{pv}}=-\mathbf{K}_{\mathrm{vp}}{ }^{T} . \mathbf{K}_{\mathrm{pp}}$ and $\mathbf{K}_{\mathrm{pf}}$ are the total pressure block and the fluid pressure diffusion operator (Laplace), respectively. Moreover, the $\mathbf{K}_{\mathrm{pp}}$ diagonal block takes values of $\left[\eta_{\phi}(1-\right.$ $\phi)]^{-1}$, physically solving the issues of the missing diagonal entries encountered in the incompressible Stokes formulation, as discussed in Räss et al. (2017a). The diagonal $\mathbf{K}_{\mathrm{ppf}}$ and $\mathbf{K}_{\mathrm{pfp}}$ blocks contain the symmetric pressure coupling terms, total-to-fluid and fluid-to-total respectively. They arise from splitting the $p_{e}$ variable (eq. 10) into total and fluid pressures. The $\mathbf{b}$ vector contains contributions from transient terms and boundary conditions.

In a linear problem, the solution vector $\mathbf{x}$ is obtained by applying the inverse of the $\mathbf{K}_{\mathrm{HM}}$ matrix to the right-hand side vector $\mathbf{b}$ :

$\mathbf{x}=\mathbf{K}_{\mathrm{HM}}{ }^{-1} \mathbf{b}$.

In the presented set of two-phase flow equations, several parameters (e.g. effective permeability, bulk viscosity) exhibit a non-linear dependence on porosity $\phi$ and effective pressure $p_{e}$. Hence the global system of equations is non-linear and a imbalance:

$\mathbf{b}-\mathbf{K}_{\mathrm{HM}} \mathbf{x}=\mathbf{f} \neq 0$.

Here $\mathbf{f}$ stands for the non-linear residual vector which quantifies the imbalance. We utilize an iterative approach to minimize the magnitude of $\mathbf{f}$. We thus update the solution vector within a nonlinear iteration cycle:

$\mathbf{x}^{k+1}=\mathbf{x}^{k}+\alpha \delta \mathbf{x}^{k+1}$,

where $\delta \mathbf{x}^{k}$ is the non-linear correction. The parameter $\alpha$ is determined in a line search procedure in order to minimize $\mathbf{f}$ :

$\min \left\|\mathbf{f}\left(\mathbf{x}^{k}+\alpha \delta \mathbf{x}^{k+1}\right)\right\|_{\mathrm{L} 2}$.

We obtain these corrections in the context of Picard and Newton iterations by evaluating the matrix $\mathbf{K}_{\mathrm{HM}}$ every iteration, thus computing the correction as:

$\delta \mathbf{x}^{k+1}=\left(\mathbf{K}_{\mathrm{HM}}{ }^{k}\right)^{-1} \mathbf{f}^{k}$.

where $k$ is the non-linear iteration index and the vector $\mathbf{f}$ is obtained as following:

$\underbrace{\left[\begin{array}{c}\mathbf{f}_{\mathrm{v}} \\ \mathbf{f}_{\overline{\mathrm{p}}} \\ \mathbf{f}_{\mathrm{p}^{\mathrm{f}}}\end{array}\right]}_{\mathbf{f}^{k}}=\underbrace{\left[\begin{array}{c}\mathbf{b}_{\mathrm{v}} \\ \mathbf{b}_{\overline{\mathrm{p}}} \\ \mathbf{b}_{\mathrm{p}^{\mathrm{f}}}\end{array}\right]}_{\mathbf{b}^{k}}-\underbrace{\left[\begin{array}{ccc}\mathbf{K}_{\mathrm{vv}}(\phi) & \mathbf{K}_{\mathrm{vp}} & \mathbf{0} \\ \mathbf{K}_{\mathrm{pv}} & \mathbf{K}_{\mathrm{pp}}\left(\phi, \bar{p}, p^{f}\right) & \mathbf{K}_{\mathrm{ppf}}\left(\phi, \bar{p}, p^{f}\right) \\ \mathbf{0} & \mathbf{K}_{\mathrm{pfp}}\left(\phi, \bar{p}, p^{f}\right) & \mathbf{K}_{\mathrm{pf}}\left(\phi, \bar{p}, p^{f}\right)\end{array}\right]}_{\mathbf{K}_{\mathrm{HM}^{k}}} \underbrace{\left[\begin{array}{c}\mathbf{v} \\ \overline{\mathbf{p}} \\ \mathbf{p}^{\mathbf{f}}\end{array}\right]}_{\mathbf{x}}$.

We here highlight the inherent non-linearities in the majority of the blocks reflecting their non-linear dependence on $\phi, \bar{p}$ and $p^{f}$. The non-linear iteration procedure needs to be performed until the magnitude of the residual decreases below a given tolerance level, $\|\left.\mathbf{f}^{k}\right|_{\mathrm{L} 2} \leq$ tol $_{\text {nonlin }}$, in order to obtain an accurate and fully coupled solution. The Picard linearization delivers a linear convergence rate that may require significantly more iterations compared to a Newton linearization. We therefore substitute the iteration matrix (eq. A7) by the Jacobian matrix $\mathbf{J}_{\mathrm{HM}}$ :

$\mathbf{J}_{\mathrm{HM}}=\frac{\partial \mathbf{f}_{i}}{\partial \mathbf{x}_{j}}$,

which contains information about gradients of the residuals with regards to the solution. We here express the Jacobian matrix as:

$\mathbf{J}_{\mathrm{HM}}=\left[\begin{array}{lll}\mathbf{J}_{\mathrm{vv}} & \mathbf{J}_{\mathrm{vp}} & \mathbf{0} \\ \mathbf{J}_{\mathrm{pv}} & \mathbf{J}_{\mathrm{pp}} & \mathbf{J}_{\mathrm{ppf}} \\ \mathbf{J}_{\mathrm{pfv}} & \mathbf{J}_{\mathrm{pfp}} & \mathbf{J}_{\mathrm{pf}}\end{array}\right]$ 

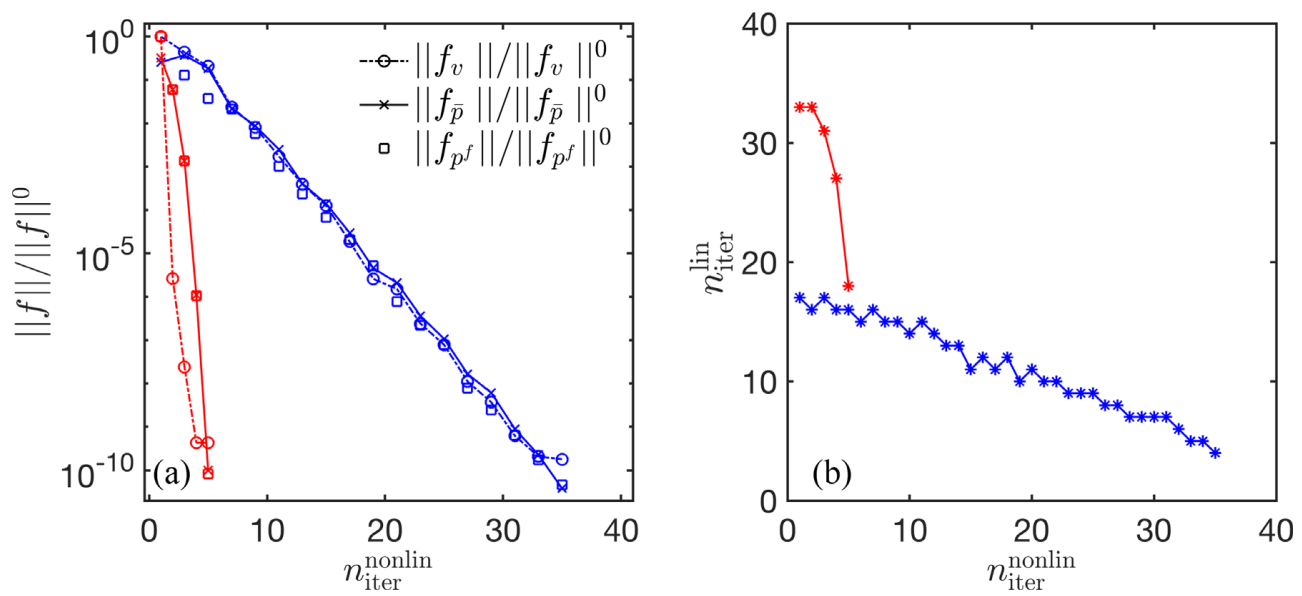

Figure A1. Comparison of Picard and Newton linearization; (a) Relative non-linear residuals as function of non-linear iterations for both the Picard (blue) and Newton (red) linearization. For enhanced readability, we only display every second non-linear iteration for the Picard residuals. (b) Number of linear iterations per non-linear step for the DI solver $n_{\mathrm{iter}}^{\text {lin }}$. The sum of linear iterations for all the non-linear steps equals 142 and 390 for the Newton and Picard linearization, respectively. This figure can be reproduced by running the HM2Di.m code distributed as supplementary material and available for download at https://bitbucket.org/lraess/m2di.

where the $\mathbf{J}$ blocks differ from the Picard $\mathbf{K}$ blocks as they are augmented with additional contributions from the gradients of porosity, total and fluid pressure as well as velocities. We finally retrieve the Newton corrections:

$\delta \mathbf{x}^{k+1}=\left(\mathbf{J}_{\mathrm{HM}}^{k}\right)^{-1} \mathbf{f}^{k}$.

and thus reach the targeted non-linear accuracy within less than 10 non-linear iterations. On the other hand, reaching similar accuracy with the Picard linearization requires about 35 iterations (Fig. A1a). Interestingly, the required number of linear iterations reported for each non-linear step (Fig. A1b) follows a quadratic and a linear trend for the Newton and the Picard linearization, respectively. Thus, the total number of linear iterations among all the non-linear steps sums up to 142 and 390 for the Newton and the Picard linearization, respectively.

\section{A2 The direct-iterative solver}

We seek to solve the following linear system:

$$
\underbrace{\left[\begin{array}{lll}
\mathbf{J}_{\mathrm{vv}} & \mathbf{J}_{\mathrm{vp}} & \mathbf{0} \\
\mathbf{J}_{\mathrm{pv}} & \mathbf{J}_{\mathrm{pp}} & \mathbf{J}_{\mathrm{ppf}} \\
\mathbf{J}_{\mathrm{pfv}} & \mathbf{J}_{\mathrm{pfp}} & \mathbf{J}_{\mathrm{pf}}
\end{array}\right]}_{\mathbf{J}_{\mathrm{HM}}} \underbrace{\left[\begin{array}{c}
\delta \mathbf{v} \\
\delta \overline{\mathbf{p}} \\
\delta \mathbf{p}^{\mathbf{f}}
\end{array}\right]}_{\delta \mathbf{x}}=-\underbrace{\left[\begin{array}{c}
\mathbf{f}_{\mathrm{v}} \\
\mathbf{f}_{\overline{\mathbf{p}}} \\
\mathbf{f}_{\mathrm{p}}
\end{array}\right]}_{\mathbf{f}} .
$$

We define the linear residuals as:

$$
\left\{\begin{array}{l}
\mathbf{r}_{\mathrm{v}}=\mathbf{f}_{\mathrm{v}}-\mathbf{J}_{\mathrm{vv}} \delta \mathbf{v}-\mathbf{J}_{\mathrm{vp}} \delta \overline{\mathbf{p}} \\
\mathbf{r}_{\overline{\mathrm{p}}}=\mathbf{f}_{\overline{\mathbf{p}}}-\mathbf{J}_{\mathrm{pv}} \delta \mathbf{v}-\mathbf{J}_{\mathrm{pp}} \delta \overline{\mathbf{p}}-\mathbf{J}_{\mathrm{ppf}} \delta \mathbf{p}^{\mathbf{f}} \\
\mathbf{r}_{\mathrm{p}^{\mathrm{f}}}=\mathbf{f}_{\mathrm{p}^{\mathrm{f}}}-\mathbf{J}_{\mathrm{pfv}} \delta \mathbf{v}-\mathbf{J}_{\mathrm{pfp}} \delta \overline{\mathbf{p}}-\mathbf{J}_{\mathrm{pf}} \delta \mathbf{p}^{\mathrm{f}}
\end{array}\right.
$$

and the solutions are found iteratively:

$$
\left\{\begin{array}{l}
\delta \mathbf{v}^{i+1}=\delta \mathbf{v}^{i}+\delta \delta \mathbf{v} \\
\delta \overline{\mathbf{p}}^{i+1}=\delta \overline{\mathbf{p}}^{i}+\delta \delta \overline{\mathbf{p}} \\
\delta \mathbf{p}^{\mathbf{f}^{i+1}}=\delta \mathbf{p}^{\mathbf{f}^{i}}+\delta \delta \mathbf{p}^{\mathbf{f}}
\end{array},\right.
$$

where $\delta \delta \mathbf{v}, \delta \delta \overline{\mathbf{p}}$, and $\delta \delta \mathbf{p}^{\mathbf{f}}$ are iterative corrections and $i$ is the iteration count.

The iterative corrections are retrieved within a two step substitution and precomputing three separate Schur complements (denoted

$$
\begin{aligned}
& \text { by } \left.^{\sim}\right): \\
& \widetilde{\mathbf{J}}_{\mathrm{pv}}=\left[\mathbf{J}_{\mathrm{pv}}-\mathbf{J}_{\mathrm{ppf}}\left(\widehat{\mathbf{J}}_{\mathrm{pf}} \mathbf{J}_{\mathrm{pfv}}\right)\right], \\
& \widetilde{\mathbf{J}}_{\mathrm{pp}}=\left[\mathbf{J}_{\mathrm{pp}}-\mathbf{J}_{\mathrm{ppf}}\left(\widehat{\mathbf{J}}_{\mathrm{pf}} \mathbf{J}_{\mathrm{pfp}}\right)\right], \\
& \widetilde{\mathbf{J}}_{\mathrm{vv}}=\left[\mathbf{J}_{\mathrm{vv}}-\mathbf{J}_{\mathrm{vp}}\left(\widehat{\mathbf{J}}_{\mathrm{pp}} \mathbf{J}_{\mathrm{pv}}\right)\right],
\end{aligned}
$$

where $\widehat{\mathbf{J}}_{\mathrm{pf}}=\operatorname{diag}^{-1}\left(\mathbf{J}_{\mathrm{pf}}\right)$ and $\widehat{\widetilde{\mathbf{J}}}_{\mathrm{pp}}=\operatorname{diag}^{-1}\left(\widetilde{\mathbf{J}}_{\mathrm{pp}}\right)$ are the approximation (diagonal preconditioner, denoted by $\widehat{\mathcal{}}$ ) of the inverse of $\mathbf{J}_{\mathrm{pf}}$ and $\widetilde{\mathbf{J}}_{\mathrm{pp}}$, respectively. The velocity as well as the total and fluid pressure corrections can be iteratively retrieved by applying the action of $\widetilde{\mathbf{J}}_{\mathrm{vv}}{ }^{-1}, \widetilde{\mathbf{J}}_{\mathrm{pp}}{ }^{-1}$ and $\mathbf{J}_{\mathrm{pf}}{ }^{-1}$, the inverse of the velocity, the total pressure and the fluid pressure Jacobian blocks, to $\widetilde{\mathbf{f}}_{\mathrm{v}}, \widetilde{\mathbf{f}}_{\overline{\mathrm{p}}}$ and $\mathbf{f}_{\mathrm{p}_{\mathrm{f}}}$ :

$$
\begin{aligned}
& \delta \delta \mathbf{v}=\widetilde{\mathbf{J}}_{\mathrm{vv}}{ }^{-1}[\underbrace{[\mathbf{f}_{\mathrm{v}}-\mathbf{J}_{\mathrm{vp}} \widetilde{\mathbf{J}}_{\mathrm{pp}}{ }^{-1} \underbrace{\left(\mathbf{f}_{\overline{\mathrm{p}}}-\mathbf{J}_{\mathrm{ppf}} \mathbf{J}_{\mathrm{pf}}^{-1} \mathbf{f}_{\mathrm{p}_{\mathrm{f}}}\right)}_{\widetilde{\mathrm{f}_{\bar{p}}}}]}_{\widetilde{\mathbf{f}_{\mathrm{v}}}}, \\
& \delta \delta \overline{\mathbf{p}}=\widetilde{\mathbf{J}}_{\mathrm{pp}}{ }^{-1}\left[\widetilde{\mathbf{f}}_{\overline{\mathrm{p}}}-\widetilde{\mathbf{J}}_{\mathrm{pv}} \delta \delta \mathbf{v}\right], \\
& \delta \delta \mathbf{p}^{\mathbf{f}}=\mathbf{J}_{\mathrm{pf}}^{-1}\left[\mathbf{f}_{\mathrm{p}^{\mathrm{f}}}-\mathbf{J}_{\mathrm{pfv}} \delta \delta \mathbf{v}-\mathbf{J}_{\mathrm{pfp}} \delta \delta \overline{\mathbf{p}}\right] .
\end{aligned}
$$

We pursue the iterations until the linear residuals reach the tolerance threshold of the linear solver $\|\mathbf{f}\|_{\mathrm{L} 2}<\operatorname{tol}_{\text {lin }}$. Since the velocity Schur complement and the fluid pressure blocks $\left(\widetilde{\mathbf{J}}_{\mathrm{vv}}\right.$ and $\left.\mathbf{J}_{\mathrm{pf}}\right)$ are symmetrical and positive-definite, we perform a Cholesky factorization of $\widetilde{\mathbf{J}}_{\mathrm{vv}}$ and $\mathbf{J}_{\mathrm{pf}}$ followed by iterative back-substitutions to compute the corrections increments (eq. A16). Since the total pressure Schur complement $\left(\widetilde{\mathbf{J}}_{\mathrm{pp}}\right)$ is a diagonal block, there is no need to perform a Cholesky factorization as its trivial inverse can readily be used in the solving procedure. In the presented examples, the fluid continuity equation is solved with Neumann boundary conditions. The subsequent null space is taken care by removing the mean of total continuity residuals at each linear and non-linear iteration.

\section{APPENDIX B: NUMERICAL TRANSIENT TIME}

The essence of the PT continuation method resides in introducing a transient or numerical time derivative to the equation of interest 
Table B1. Number of PT iteration as function of damping parameter $v_{\mathrm{v}}$ (utilized in [1- $\left.v_{\mathrm{v}} / n_{i}\right]$ ) for the 3-D hydromechanical solver. The resolution used is $1014^{3}$ and the targeted non-linear tolerance is tol $\mathrm{n}_{\text {nonlin }}=10^{-8} \cdot v_{\mathrm{p}^{\mathrm{f}}}=0.9$ is kept constant for this investigation.

\begin{tabular}{lcccccccccc}
\hline$\nu_{\mathrm{v}}$ & 2 & 3 & 4 & 5 & 6 & 7 & 8 & 9 & 10 & 20 \\
\hline \# of PT iterations $\left(\times 10^{-4}\right)$ & 52.60 & 6.65 & 5.50 & 5.90 & 6.15 & 7.05 & 7.15 & 7.60 & 8.45 & 14.55 \\
\hline
\end{tabular}

Table B2. Number of PT iteration as function of damping parameter $v_{\mathrm{p}^{\mathrm{f}}}$ for the 3-D hydromechanical solver. The resolution used is $1014^{3}$ and the targeted non-linear tolerance is tol $\mathrm{l}_{\text {nonlin }}=10^{-8} \cdot v_{\mathrm{v}}=4$ is kept constant for this investigation.

\begin{tabular}{lcccccrrr}
\hline$\nu_{\mathrm{p}^{\mathrm{f}}}$ & 0.50 & 0.60 & 0.70 & 0.80 & 0.88 & 0.90 & 0.92 & 0.94 \\
\hline$\#$ of PT iterations $\left(\times 10^{-4}\right)$ & $>100$ & $>100$ & 13.85 & 9.65 & 6.35 & 5.50 & 5.50 & 5.75 \\
\hline
\end{tabular}

and to iterate on until steady state is reached. At that stage, the time derivative vanishes and the equation is solved. The following generic 1-D example illustrates this basic workflow. Assuming one must find the solution to the elliptic problem:

$C=\frac{\partial^{2} A}{\partial x^{2}}$,

without performing a direct solve. The first step is to rearrange the equation, sending all the terms to the right-hand side, assembling the residual:

$0=\frac{\partial^{2} A}{\partial x^{2}}-C=f_{\mathrm{A}}$.

It is now possible to introduce a numerical time derivative on the left-hand side of the equation (eq. B2):

$\frac{\partial A}{\partial \tau}=f_{\mathrm{A}}$,

and use it to iteratively reach the steady state of the system:

$A^{k}=A^{k-1}+\Delta \tau f_{\mathrm{A}}{ }^{k}$,

where $k$ and $\Delta \tau$ are the pseudo-transient iteration count and timestep, respectively. Once $A$ stops changing over successive iterations, the numerical time derivative vanishes. Thus, the residual $\mathrm{f}_{\mathrm{A}}$ has converged towards machine precision, and the eq. (B1) is solved. Notably, first, the method is also applicable if $\mathrm{f}_{\mathrm{A}}$ contains non-linear terms - as present in the two-phase flow equations described here. Second, the solution of parabolic equations using the PT continuation leads to an implicit solution, since both the temporal and the spatial derivative are included in the residual. The solution of the unknown field satisfies the equation at time $(t+\Delta t)$. Additional details regarding the PT continuation approach can be found in selected contributions (e.g. Kelley \& Liao 2013; Kelley \& Keyes 1998, and references therein).

Moreover, the utilization of a second order PT scheme (Frankel 1950) permits to efficiently damp the residuals of the momentum (Table B1) and fluid pressure (Table B2) equations. As results, the number of total iterations needed to reach the desired tolerance is greatly reduced.

\section{APPENDIX C: SOLITARY WAVES BENCHMARK}

We perform two validations of our numerical implementation. First, we compare numerical and exact analytical 1-D solutions. Second, we verify the convergence of 2-D numerical solutions with growing spatial and temporal resolutions. Both 1-D exact solution and converged 2-D numerical solutions are proposed as benchmark cases for validation of hydromechanical coupling in numerical codes.

\section{C1 Analytical 1-D solution}

We derive a general 1-D analytical solution using the hydraulic potential approach (e.g. Yarushina et al. 2015b; Connolly \& Podladchikov 2014). Using a coordinate system moving together with the 1-D propagating porosity wave, one can exclude depth from the compaction equations and express effective pressure as a function of porosity. Thus, the governing system of coupled partial differential equations (eq. 1-4, 6) is simplified to a system of ordinary differential equations:

$$
\begin{aligned}
\frac{\partial v_{z}^{s}}{\partial z} & =v_{\mathrm{pw}} \frac{\partial \log (1-\phi)}{\partial z}, \\
\frac{\partial\left[\phi\left(v_{k}^{f}-v_{k}^{s}\right)\right]}{\partial z} & =-v_{\mathrm{pw}} \frac{\partial \log (1-\phi)}{\partial z}, \\
\frac{\partial \bar{p}}{\partial z} & =-\bar{\rho} g, \\
\phi\left(v_{i}^{f}-v_{i}^{s}\right) & =-\frac{k_{0}}{\mu^{f}}\left(\frac{\phi}{\phi_{0}}\right)^{n_{k}}\left(\frac{\partial\left(\bar{p}-p_{e}\right)}{\partial z}+\rho^{f} g\right), \\
\frac{\partial v_{z}^{s}}{\partial z} & =-\frac{\phi p_{e}}{\phi_{0} \eta_{C}(1-\phi)},
\end{aligned}
$$

where $v_{\mathrm{pw}}$ is the porosity wave velocity with respect to the solid and $z$ is the vertical coordinate. In the present case, we consider all non-linearities previously introduced and assume here that the total pressure is obtained by the integral of $\bar{\rho} g z$ with depth. Eliminating the total pressure and the fluid and solid velocities leads to the following system:

$$
\begin{aligned}
-\frac{\phi p_{e}}{\phi_{0} \eta_{C}(1-\phi)} & =v_{\mathrm{pw}} \frac{\partial \log (1-\phi)}{\partial z}, \\
\frac{\partial\left[-\frac{k_{0}}{\mu^{f}}\left(\frac{\phi}{\phi_{0}}\right)^{n_{k}}\left(-\frac{\partial p_{e}}{\partial z}-(1-\phi)\left(\rho^{s}-\rho^{f}\right) g\right)\right]}{\partial z} & =-v_{\mathrm{pw}} \frac{\partial \log (1-\phi)}{\partial z} .
\end{aligned}
$$

We finally integrate and re-arange the second equation:

$$
\begin{aligned}
\frac{\partial \log (\phi)}{\partial z}= & \frac{p_{e}}{\phi_{0} \eta_{C} v_{\mathrm{pw}}}, \\
\frac{\partial p_{e}}{\partial z}= & \left(-(1-\phi)\left(\rho^{s}-\rho^{f}\right) g\right) \\
& -\frac{\mu^{f}}{k_{0}}\left(\frac{\phi_{0}}{\phi}\right)^{n_{k}}\left(v_{\mathrm{pw}} \log (1-\phi)+q_{0}\right),
\end{aligned}
$$

where $q_{0}$ is an integration constant. This system of two ordinary equations can be solved analytically and visualized on a phase plane (Fig. C1c). The phase trajectories represented on the $\left(\phi, p_{e}\right)$ plane characterize the type of solution (Yarushina et al. 2015b). The vectors stand for the gradients $\partial \log (\phi) / \partial z$ and $\partial p_{e} / \partial z$ and are tangential to the trace of the stationary solution (phase vectors - Fig. C1c). The 


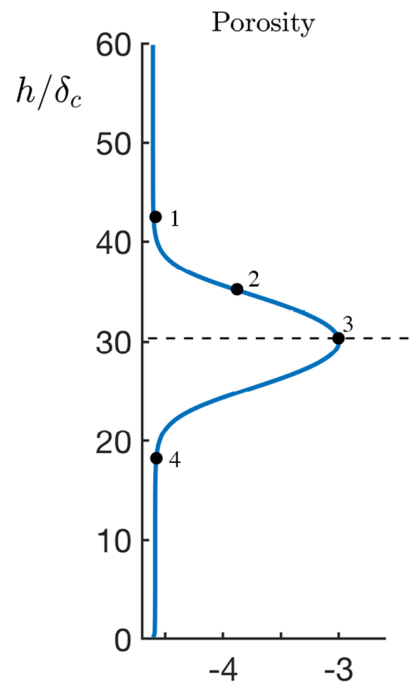

(a) $\log _{10}(\phi)$

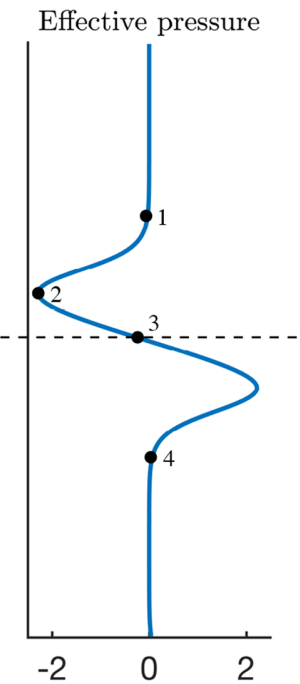

(b) $p_{e}$

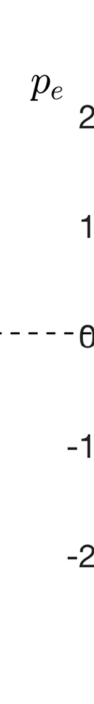

Phase portrait

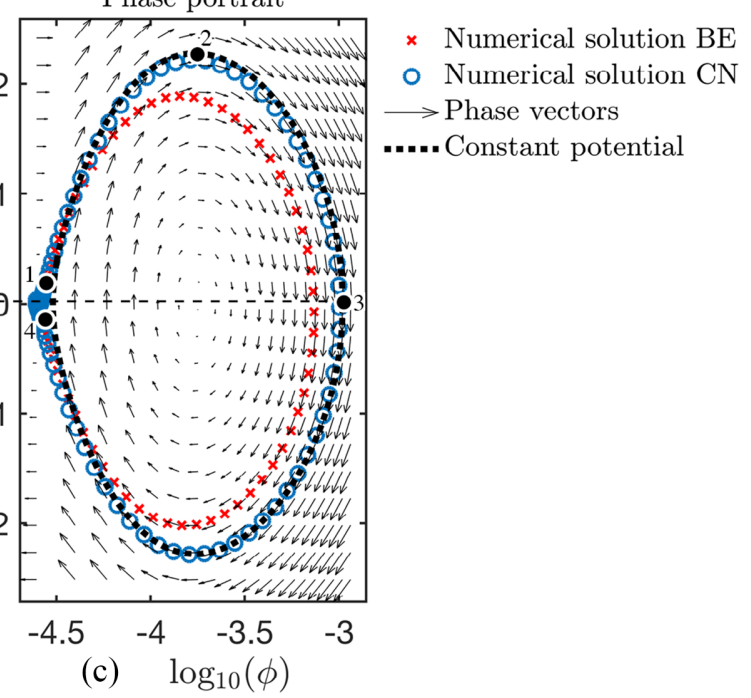

Figure C1. Stationary 1-D solution of (a) porosity, (b) effective pressure and (c) the phase portrait reported both for the $1^{\text {st }}$ and $2^{\text {nd }}$ order time discretization schemes, Backward Euler (BE) and Crank-Nicolson (CN), respectively. The phase portrait is analogous to Fig. 4 in Yarushina et al. (2015b) and depicts: (1) background porosity and effective pressure levels, (2) decompaction regime associated with a negative effective pressure, (3) maximal amplitude of the porosity anomaly (also represented by the horizontal dashed line) and (4) back to background porosity and effective pressure level within the compaction regime.

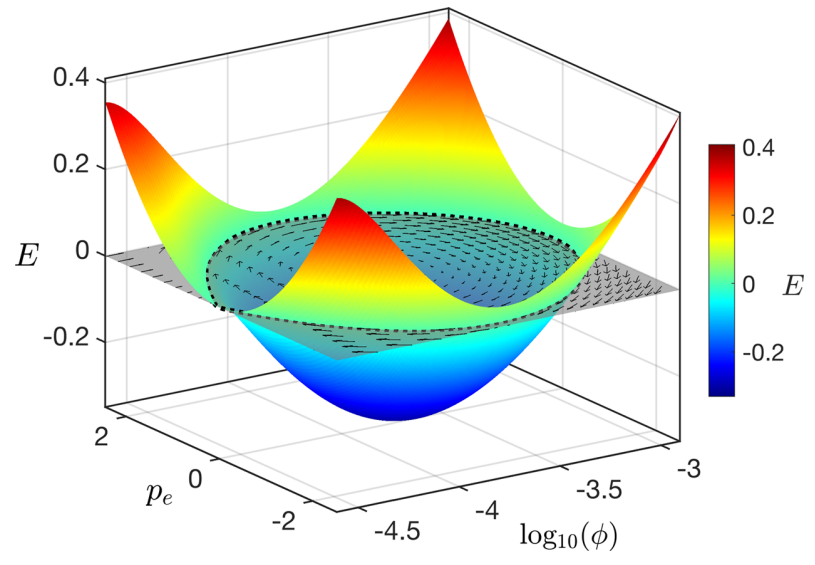

Figure C2. Potential surface $E\left(\phi, p_{e}\right)$ (coloured parabolic surface) intersecting the hydraulic potential plane $H(\phi)$ (grey plane). The intersection of the two surfaces produces the constant potential (dashed line) used in Fig. $\mathrm{C} 1(\mathrm{c})$. The arrows on the $H(\phi)$ plane stand for the phase vectors and we use the potential surface $E\left(\phi, p_{e}\right)$ values as colormap.

1-D numerical stationary porosity (Fig. C1a) and effective pressure (Fig. C1b) profile are reported on the $\left(\phi, p_{e}\right)$ plane (numerical solution - Fig. C1c). We then compare our numerical solution to the analytically obtained trajectory (constant potential - Fig. C1c). This analytical trajectory is obtained by the intersection of $E\left(\phi, p_{e}\right)$ and $\left[H(\phi)-H\left(\phi_{0}\right)\right]$ and represents the potential function $E\left(\phi, p_{e}\right)$ at the zero level (grey plane - Fig. C2) of the hydraulic potential function $H(\phi)$ :

$E\left(\phi, p_{e}\right)=\frac{p_{e}^{2}}{2 \phi_{0} \eta_{C} v_{\mathrm{pw}}}+\left[H(\phi)-H\left(\phi_{0}\right)\right]$,

where

$H(\phi)=-\int \frac{\partial p_{e}}{\partial z} d \log (\phi)$.

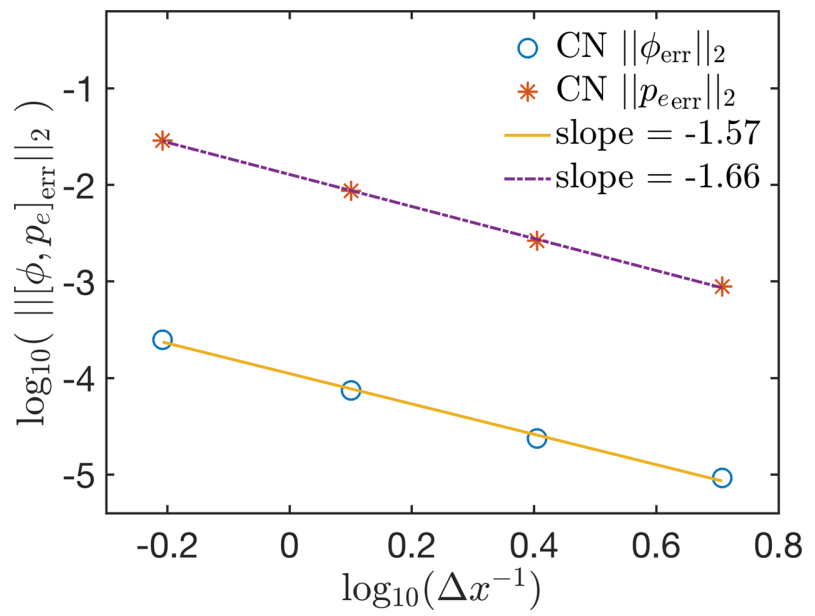

Figure C3. Spatial convergence test results. We report errors (L2 norm) of porosity and effective pressure relative to a 2-D reference high-resolution simulation for various grid spacings $\Delta x^{-1}$. All results are obtained using a second order Crank-Nicolson (CN) time-integration scheme. The continuous lines stand for the linear fit of the plotted data, with slopes of close to -1.5 .

For a cubic permeability power-law exponent $\left(n_{k}=3\right)$ the above integral evaluates to an explicit function of porosity:

$$
\begin{aligned}
H(\phi)= & -\frac{v_{\mathrm{pw}} \mu^{f}}{3 k_{0}} \phi_{0}{ }^{3}\left(\frac{1}{2} \phi^{-2}+\phi^{-1}-\ln (\phi)+\frac{\ln \left(1-\phi_{0}\right)}{\phi^{3}}\right. \\
& \left.-\frac{(1-\phi) \ln (1-\phi)\left(3 \phi+(1-\phi)^{2}\right)}{\phi^{3}}\right) \\
& -\left(\rho^{s}-\rho^{f}\right) g\left(\phi-\ln (\phi)+\frac{1}{3} \frac{\phi_{0}^{3}\left(\phi_{0}-1\right)}{\phi^{3}}\right)
\end{aligned}
$$

Our numerical solution shows a relatively good match with the analytically obtained trajectory (Fig. C1c) which is obtained by the intersection of $E\left(\phi, p_{e}\right)$ and $\left[H(\phi)-H\left(\phi_{0}\right)\right]$. We capture well the regions of highest amplitude (3) and both the decompaction (2) and 


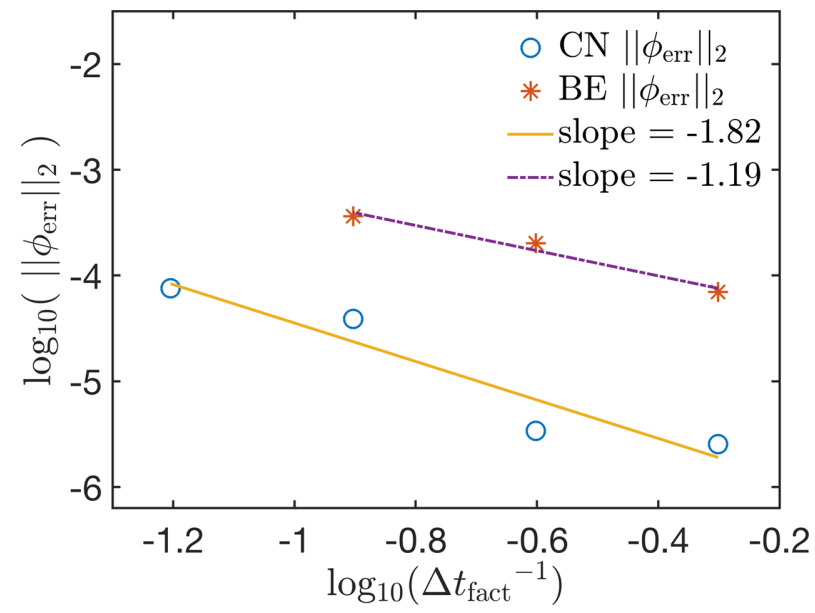

Figure C4. Temporal convergence test results. We report errors (L2 norm) of porosity relative to a 2-D reference high temporal resolution simulation $\left(\Delta t_{\text {fact }}=1\right)$ for various multiple of the high-resolution time step. Results are obtained using either a first order Backward-Euler (BE) or a second order Crank-Nicolson $(\mathrm{CN})$ accurate time-integration scheme. The continuous lines stand for the linear fit of the plotted data; the $\mathrm{CN}$ schemes tackles quadratic convergence while the BE scheme is close to linear trend. The BE tests failed with the largest tested value of $\Delta t_{\text {fact }}=16$.

compaction regimes. However, our numerical solutions shows some discrepancy in the regions where pores start to expand (1) or are very close to recover their original fluid content (4). We utilized a numerical resolution of 200 gridpoints in the vertical direction $h$, and five girdpoints in the $x$ direction to achieve a pseudo 1-D configuration. We rely on a second order in time $\mathrm{CN}$ discretization and recorded the numerical data once we obtained the stationary solution (in a moving coordinate frame). In comparison, a Backward Euler scheme (first order) captures with less accuracy the compaction phase, and fits thus not as well the analytical contour (Fig. C1c).

\section{C2 Convergence verification in 2-D}

As a second validation, we report the convergence of our numerical implementation for the full set of non-linear coupled two-phase equations with both spatial and temporal resolution. We consider a numerical domain extend of $50 \delta_{c}$ and $100 \delta_{c}$ in $x$ and $y$ directions, respectively. The background initial porosity is $\phi_{0}=10^{-2}$ and we locate a Gaussian anomaly at $0.2 L_{y}$ with an amplitude equal to $3 \phi_{0}$ and a standard deviation of $6 \delta_{c}$. The total dimensionless simulation time is $0.1 \tau_{c}$, which permits the porosity anomaly to propagate upwards about 80 per cent of the domain depth.

In first, we vary the numerical gird resolution keeping the grid step in $x$ and $y$ direction equal to each other $(\Delta x=\Delta y)$. We utilize a high-resolution simulation as reference and perform 4 additional simulations where we keep dividing the number of gridpoints in both $x$ and $y$ direction by a factor 2 . We report the L 2 norms:

$$
\begin{aligned}
\left\|\phi_{\text {err }}\right\|_{2} & =\left\|\phi_{\text {ref }}-\phi_{\text {coarse }}\right\|_{2}, \\
\left\|p_{e_{\text {err }}}\right\|_{2} & =\left\|p_{e_{\text {ref }}}-p_{e_{\text {coarse }}}\right\|_{2},
\end{aligned}
$$

for both porosity $\phi$ and effective pressure $p_{e}$ as a function of the grid size on a logarithmic plot (Fig. C3). In this test we rely on a second order time discretization using a $\mathrm{CN}$ scheme. Our numerical implementation convergences with decreasing grid resolution and we report linear fitting slopes of about -1.5 for both the porosity and effective pressure L2 norms.

In second, we vary the numerical time step $\Delta t$ while keeping the numerical grid resolution fixed. We investigate the impact of multiplying by a factor $\Delta t_{\text {fact }}$ the reference time step (high temporal resolution). We report the L2 norms for errors in the porosity field (Fig. C4) defined as:

$(\mathrm{CN}, \mathrm{BE})\left\|\phi_{\mathrm{err}}\right\|_{2}=\left\|\phi_{\text {ref }}-\phi_{\left(\Delta \mathrm{t} \Delta \mathrm{t}_{\mathrm{fact}}\right)}\right\|_{2}$,

where $\mathrm{CN}$ and $\mathrm{BE}$ refer to the second order temporal discretization using the $\mathrm{CN}$ scheme and the first order Backward-Euler (BE) scheme, respectively. We report close to quadratic convergence of the porosity error for the $\mathrm{CN}$ implementation, while the $\mathrm{BE}$ scheme converges with a slope close to 1 . Also, the BE method fails to converge the largest tested value of $\Delta t_{\text {fact }}=16$ while the $\mathrm{CN}$ scheme resolves it. This is accordance with the observations reported by Duretz et al. (2019) for thermomechanical couplings. This behaviour reflects that the non-linearities arising from the multiphysics coupling significantly impact the allowed maximal physical time step selection.

\section{APPENDIX D: THE PERFORMANCE OF THE SOLVERS}

We use two distinct metrics to report the solvers' performance, the effective memory throughput $\left(\mathrm{MTP}_{\text {effective }}\right)$ and the wall-time. We first evaluate both the MATLAB CPU-based and the CUDA C GPU-based 2-D PT solver implementations in terms of $\mathrm{MTP}_{\text {effective }}$. We then compare the 2-D GPU-based PT solver to the MATLABbased HM2Di DI solver using the wall-time metric to time the convergence of one non-linear time-step.

The memory accesses rather than the floating-point operations per second $\left(\mathrm{FLOP} \mathrm{s}^{-1}\right.$ ) limit the performance of the PT algorithm, which is designed to perform stencil operation in a matrix-free approach. For this reason, we chose the $\mathrm{MTP}_{\text {effective }}$ metric (Omlin 2017) to evaluate how efficiently data is transferred between the memory and the computation units, in gigabytes per second $\left(\mathrm{GB} \mathrm{s}^{-1}\right)$ :

$\mathrm{MTP}_{\text {effective }}=\frac{n_{i}^{\text {tot }} n_{\mathrm{t}} n_{\mathrm{IO}} n_{\mathrm{p}}}{2^{30} t_{\mathrm{n}_{\mathrm{t}}}}$,

where $n_{i}^{\text {tot }}$ is the total grid resolution, $n_{\mathrm{t}}$ is the number of time-steps or iterations performed, $n_{\mathrm{IO}}$ is the number of memory accesses performed, $n_{\mathrm{p}}$ is the floating-point precision (either 4 or 8 bytes), and $t_{\mathrm{n}}$ is the time in seconds needed to perform the $n_{\mathrm{t}}$ steps. The number of memory accesses $\left(n_{\mathrm{IO}}\right)$ defines the minimum number of read-and-write or read-only operations required to solve the specific physics. For 2-D coupled hydromechanics, the read-and-write operations correspond to the updates of the DoFs $\left(v_{x}, v_{y}, \bar{p}, p^{f}\right)$, and three additional read-only operations for converging the non-linear viscosity; in our case, $n_{\mathrm{IO}}=11$.

The performance benchmark runs are performed using doubleprecision floating-point arithmetic ( $n_{\mathrm{p}}=8$ bytes) for a fair comparison in particular between MATLAB and CUDA C implementations. However, we also report $\mathrm{MTP}_{\text {effective }}$ using single-precision arithmetic for the CUDA C PT solver implementation ( $n_{\mathrm{p}}=4$ bytes). One motivation is the identical accuracy of the converged results (Figs 4 and 5) for twice lower memory usage and twice faster exe-

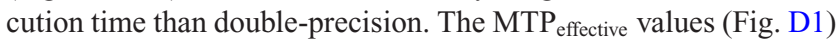
represent the efficiency of memory access for both the vectorized MATLAB CPU and CUDA C single GPU PT solver implementations. The reported numbers are to be compared to the peak memory 
Table D1. Parallel efficiency of the 3-D GPU MPI hydromechanical PT solver for 1-128 MPI process. Each MPI process handles one Nvidia Titan X GPU. The local problem size solved on each of the GPUs contains $255^{3}$ gridpoints.

\begin{tabular}{|c|c|c|c|c|c|}
\hline \# of MPI processes & 1 & 8 & 27 & 64 & 128 \\
\hline Numerical 3-D grid resolution & $255^{3}$ & $508^{3}$ & $761^{3}$ & $1014^{3}$ & $1277^{3}$ \\
\hline Time for 100 iteration [s] & 5.78 & 5.84 & 5.97 & 6.03 & 6.03 \\
\hline Parallel efficiency [-] & 1.00 & 0.99 & 0.97 & 0.96 & 0.96 \\
\hline
\end{tabular}

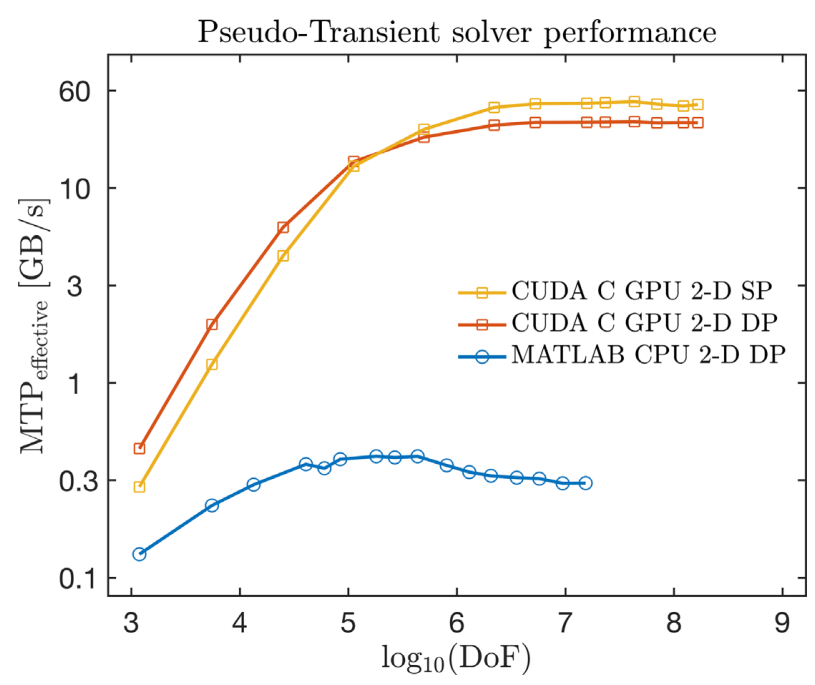

Figure D1. Performance evaluation of the PT-based hydromechanically coupled solvers in terms of effective memory throughput $\mathrm{MTP}_{\text {effective }}$ in $\mathrm{GB} \mathrm{s}^{-1}$. We compare the 2-D MATLAB-based implementation running on an Intel i5 (2016) processor (CPU) with 32 GB of RAM with the 2-D GPUbased implementations using both SP and DP arithmetic, respectively. The GPU is a Nvidia Titan Xp (Pascal) with 16 GB of on-chip RAM. The DoF represents 4 variables in 2-D $\left(v_{x}, v_{y}, \bar{p}\right.$ and $\left.p^{f}\right)$ multiplied by the respective number of gridpoints.

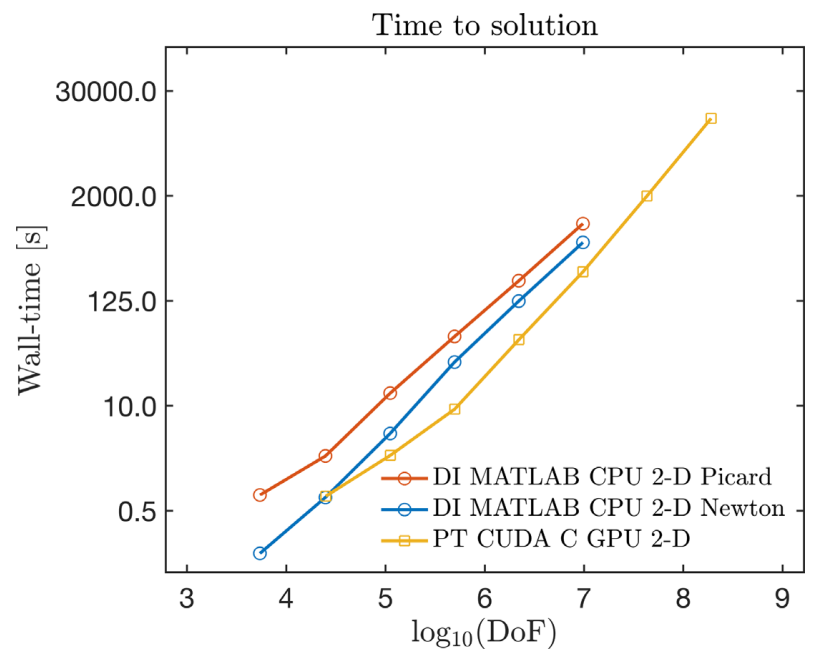

Figure D2. Performance evaluation of the hydromechanically coupled solvers in terms of time-to-converge (in seconds) one non-linear step to tol $l_{\text {nonlin }}=10^{-8}$ (wall-time). We compare the PT GPU-based solver (CUDA C) to the DI CPU (MATLAB) based solver for both the Newton and the Picard linearization, respectively. We report only DP arithmetic timings.

throughput values $\left(\mathrm{MTP}_{\text {peak }}\right)$ for the specific hardware, here an In-

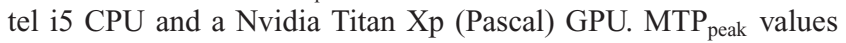
are measured performing memory copy only, without any computation. Values of $\mathrm{MTP}_{\text {peak }}$ are in the order of $20 \mathrm{~GB} \mathrm{~s}^{-1}$ for the Intel i5 CPU and in the order of $390 \mathrm{~GB} / \mathrm{s}$ for the Titan Xp GPU. The MATLAB CPU implementation runs at about 2 per cent of the
$\mathrm{MTP}_{\text {peak }} \mathrm{CPU}\left(20 \mathrm{~GB} \mathrm{~s}^{-1}\right)$, while the CUDA C GPU codes performs at about 15 per cent of the $\mathrm{MTP}_{\text {peak }}$ GPU $\left(390 \mathrm{~GB} \mathrm{~s}^{-1}\right)$. The overall performance gain of the parallel GPU implementation versus the serial CPU routines is more than two orders of magnitude. Some optimization steps are still possible, to bring the GPU MTP effective values closer to $\mathrm{MTP}_{\text {peak }}$ values. Such considerations are beyond the scope of this study, but may include an increased number of on-thefly computations, kernel rearranging and register queues (Omlin 2017).

The effective memory throughput metric reports the efficiency of hardware utilization for a specific implementation of the hydromechanical solver. But to compare the memory-bounded stencil PT iterative approach to the DI solver HM2Di, we use a more relevant measure. Thus, we chose the wall-time metric to assess the overall time-to-solution of a single non-linear step converged to tol $_{\text {nonlin }}=10^{-8}$ (Fig. D2). The DI solver shows a close to linear increase of wall-time with increasing problem size (DoF). It is implemented in MATLAB and we use an Intel i5 (2016) CPU on a system equipped with 32 GB of RAM for computations. The maximal 2-D problem size fitting in 32 GB RAM represent a numerical domain of $1024 \times 2048$ gridpoints, solved in a wall-time of $10 \mathrm{~min}$ (Newton linearization) and in $16 \mathrm{~min}$ (Picard linearization). In comparison, only 4.5 min were needed to converge the same problem using the CUDA C GPU-based PT solver, on a Nvidia Titan Xp (Pascal) accelerator (Fig. D2). The speedup versus the DI Newton implementation is about a factor 2. The PT method's additional key benefit is the maximal problem size that can be resolved using the available 12 GB of GPU on-chip RAM; $134 \mathrm{MDoF}$ represent a numerical 2-D domain size of $4096 \times 8192$ gridpoints. On all tested resolutions, the GPU implementation of the PT method outperforms both the Newton and Picard DI solver (HM2Di) in terms of wall-time. Further, for fair comparison, we realized the study using double-precision arithmetic calculation on the GPU. Moving towards single-precision arithmetic, a domain that includes twice the number of gridpoints may fit in the $12 \mathrm{~GB}$ of on-chip GPU RAM, and computation efficiency is enhanced, since the GPU's compute chip is largely populated with single-precision arithmetic units. However, the calculations would not allow one to reach the target accuracy of tol $\mathrm{l}_{\text {nonlin }}=10^{-8}$.

One additional benefit of the matrix-free PT solvers is their ability to scale on MPI implementations targeting distributed memory machines. We report efficient MPI implementation of our 3-D hydromechanical PT solver, resulting in a parallel efficiency close to 1.0 on more than 128 GPUs (Table D1). The parallel efficiency reports the ratio of the execution time required to perform a given number of iterations by the execution time obtained on a single process run (no communication). We achieve this successful scaling since only local boundaries need to be exchanged among neighbouring processes using MPI point-to-point communication; MPI processes featuring internal boundaries receive their boundary conditions from overlapping neighbouring processes computed nodes. Moreover, we utilize some asynchronous execution CUDA features in order to execute the MPI communication while computing the internal nodes of each local problem resulting in hiding all MPI communications by computations. 